\title{
Carbon Dioxide Information Analysis Center: FY 1992 Activities
}

\author{
Robert M. Cushman \\ and \\ Frederick W. Stoss* \\ Carbon Dioxide Information Analysis Center
}

Environmental Sciences Division

Publication No. 3984

Date Published: March 1993

*Energy, Environment, and Resources Center

The University of Tennessee-Knoxville

Prepared for the

Global Change Research Program

Environmental Sciences Division

Office of Health and Environmental Research

U.S. Department of Energy

Budget Activity Number KP 0500000

Prepared by the

Carbon Dioxide Information Analysis Center

Oak Ridge National Laboratory

Oak Ridge, Tennessee 37831-6335

managed by

Martin Marietta Energy Systems, Inc. for the

U.S. Department of Energy

under contract DE-AC05-84OR21400 


\section{Table cf Contents}

List of Figures $\ldots \ldots \ldots \ldots \ldots \ldots \ldots \ldots \ldots \ldots \ldots \ldots \ldots \ldots \ldots \ldots \ldots \ldots$

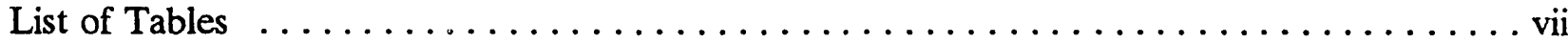

Acknowledgments $\ldots \ldots \ldots \ldots \ldots \ldots \ldots \ldots \ldots \ldots \ldots \ldots \ldots \ldots \ldots \ldots \ldots$

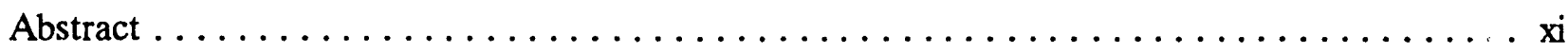

Introduction $\ldots \ldots \ldots \ldots \ldots \ldots \ldots \ldots \ldots \ldots \ldots \ldots \ldots \ldots \ldots \ldots \ldots \ldots \ldots \ldots$

Highlights $\ldots \ldots \ldots \ldots \ldots \ldots \ldots \ldots \ldots \ldots \ldots \ldots \ldots \ldots \ldots \ldots \ldots \ldots \ldots \ldots$

Organization and $\operatorname{Staff} \ldots \ldots \ldots \ldots \ldots \ldots \ldots \ldots \ldots \ldots \ldots \ldots \ldots \ldots \ldots$

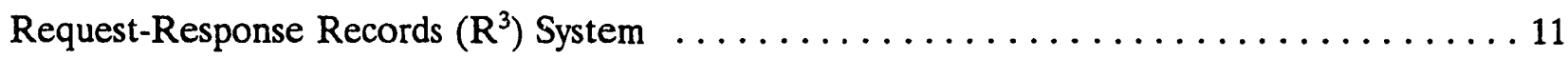

World Directory of $\mathrm{CO}_{2}$ Researchers and Policymakers $\ldots \ldots \ldots \ldots \ldots \ldots \ldots$

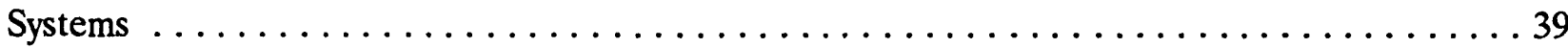

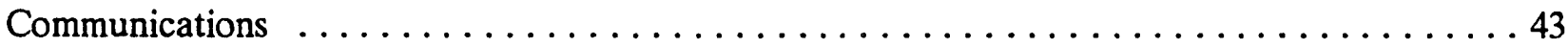

Collaborative Efforts with China $\ldots \ldots \ldots \ldots \ldots \ldots \ldots \ldots \ldots \ldots \ldots \ldots \ldots$

Numeric Data and Computer Model Packaging $\ldots \ldots \ldots \ldots \ldots \ldots \ldots \ldots \ldots \ldots \ldots \ldots \ldots$

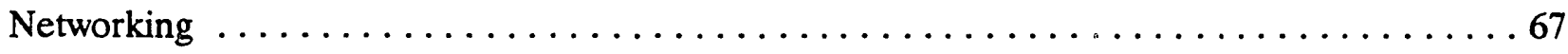

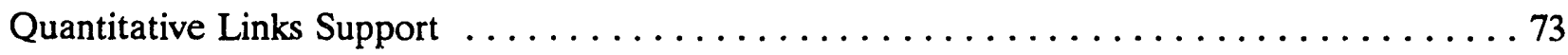

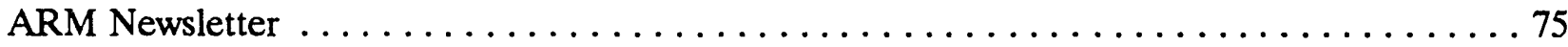




\section{List of Figures}

Figure

1. CDIAC produced 200 copies of its first CD-ROM, which stores about 217,000

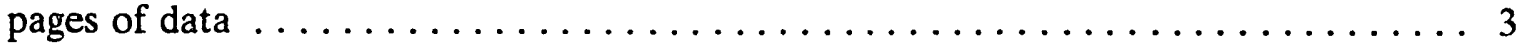

2. Locations of the more than $\mathbf{7 5 0 0}$ precipitation stations contained in the Global Historical Climatology Network data base $\ldots \ldots \ldots \ldots \ldots \ldots \ldots \ldots$

3. The first dual-language document in the series, Selected Translated Abstracts of Russian-Language Climate-Change Publications: I. Surface Energy Budget ........6 6

4. CDIAC organization (September 30,1992$) \ldots \ldots \ldots \ldots \ldots \ldots \ldots$

5. FY 1992 request/response activities world map $\ldots \ldots \ldots \ldots \ldots \ldots \ldots$

6. FY 1992 request/response activities pie chart $\ldots \ldots \ldots \ldots \ldots \ldots \ldots \ldots$

7. Request/response activities world map for FY 1985 through FY $1992 \ldots \ldots \ldots$

8. Request/response activities pie chart for FY 1985 through FY $1992 \ldots \ldots \ldots$

9. Request/response activities graph for FY 1985 through FY $1992 \ldots \ldots \ldots \ldots \ldots$

10. Countries represented in the World Directory $\ldots \ldots \ldots \ldots \ldots \ldots \ldots \ldots \ldots$

11. World Directory affiliations for FY $1992 \ldots \ldots \ldots \ldots \ldots \ldots \ldots \ldots \ldots \ldots \ldots \ldots \ldots$

12. FY 1992 requests for numeric data and computer model packages $\ldots \ldots \ldots \ldots 2$

13. FY 1985 through FY 1992 requests for numeric data and computer

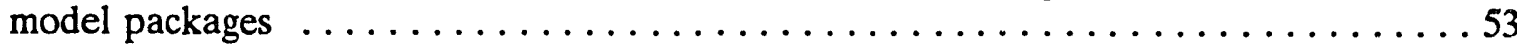

14. Numeric data packages, computer model packages, and Trends on diskette activities for FY 1985 through FY 1992 


\section{List of Tables}

Table

Page

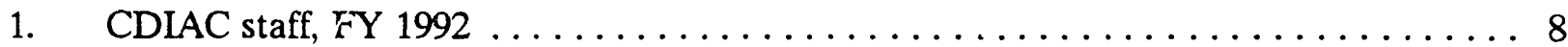

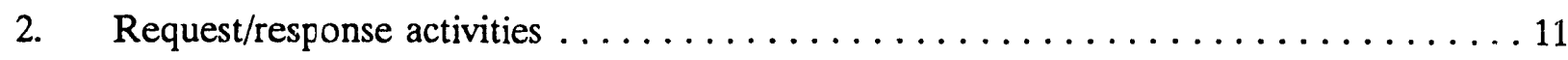

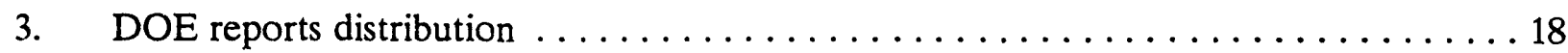

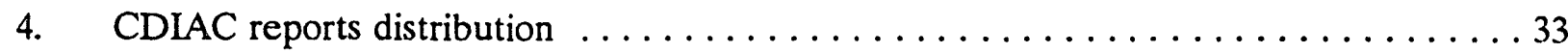

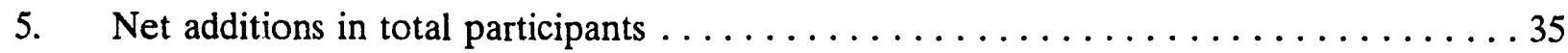

6. Alphabetical listing of countries and their outlying territories $\ldots \ldots \ldots \ldots$

7. Numeric data package/computer model package requests $\ldots \ldots \ldots \ldots \ldots$

8. Requests for documents + tape/diskette and documents only $\ldots \ldots \ldots \ldots \ldots \ldots 1$ 


\section{Acknowledgments}

As with any collective effort, this report has been prepared with the help of many people. CDIAC's greatest debt is to the contributing investigators and researchers whose data compose the core of CDIAC's information analysis and data packaging. We also extend our thanks to the laboratories, data centers, agencies, institutions, and organizations that have supported the efforts of this research community. Were it not for the voluntary cooperation and dedication of this group of researchers, CDIAC, as well as its derived information products, comprehensive information services, and specialty publications, could not exist.

Our thanks are also extended to the thousands of people from around the world who have sought assistance from CDIAC over the past year. CDIAC was founded with the intent of providing broad information services to a multidisciplinary audience with divergent interests and needs. We continue to be delighted not only with the response received from the research community for these services but also by the increase in requests for CDIAC services by policy and social scientists, educators, and others interested in various aspects of global environmental issues, including climate change.

We would like to express our gratitude to the following personnel in CDIAC at Oak Ridge National Laboratory and Martin Marietta Energy Systems, Inc. Their collective efforts have brought this report into being. Our deepest thanks go to Sonja Jones, Laura Morris, Debbie Shepherd, and Tim Stamm, who handle information requests and respond to requests for CDIAC's publications, numeric data packages, and computer model packages, and whose record keeping serves as the core for producing the statistics reported in this document. We also thank Marvel Burtis for her specialized desktop publishing, design, and production skills. We acknowledge the work of Rich Daniels, Dale Kaiser, Bob Sepanski, Russ Vose, and Tammy White, who, under the supervision of Tom Boden, are responsible for the detailed information analysis and packaging of numeric data. We thank Gregg Marland, who-with Tom Boden-annually synthesizes the data from various sources into the global and country-by-country $\mathrm{CO}_{2}$ emissions data base that is so important to the global-change community. And we thank Don Lue and David Sill, who maintain CDIAC's computing system, under Tommy Nelson's direction. Finally, we thank Patty Crabtree, CDIAC secretary, and Penny Harmon, CDIAC telephone receptionist, for keeping the office paperwork and telephone traffic flowing smoothly. Sadly, we note that Jody Dalton and Helen Graves have left CDIAC for other pursuits.

We express also our thanks to Tom Gross, program manager with the U.S. Department of Energy's Office of Health and Environmental Research, Environmental Sciences Division, Global Change Research Program. Tom has long supported international exchange of scientific information among an audience with multidisciplinary interests. His visionary promotion of the value-added process of providing information has been a mainstay in developing CDIAC's services and derived-information products. We thank Ari Patrinos, director of the DOE Environmental Sciences Division, and program managers Roger Dahlman and Mike Riches for their continued support of CDIAC activities.

We also thank Paul Kanciruk, former CDIAC director and currently manager of the Environmental Information Analysis Program in the Environmental Sciences Division (ESD), at Oak Ridge National Laboratory (ORNL); Fran Sharples, head of the ORNL ESD Environmental Analyses Section; and Bob Van Hook, director of ORNL ESD, for their constant encouragement.

CDIAC is supported by the U.S. Department of Energy's Office of Health and Environmental Research, Environmental Sciences Division, Global Change Research Program. CDIAC is housed in the Envirunmental Sciences Division at Oak Ridge National Laboratory, which is managed by Martin Marietta Energy Systems, Inc., for the U.S. Department of Energy under Contract DE-AC05-84OR21400. Carbon Dioxide Information Analysis Center: FY 1992 Activities is ORNL Publication Number ORNL/CDIAC-58 and is ESD Publication No. 3984. 


\begin{abstract}
CUSHMAN, R. M. and F. W. STOSS. 1993. Carbon Dioxide Information Analysis Center: FY 1992 Activities, ORNL/CDIAC-58, Oak Ridge National Laboratory, Oak Ridge, Tennessee. 89 pp.

During the course of a fiscal year, Oak Ridge National Laboratory's Carbon Dioxide Information Analysis Center (CDLAC) distributes thousands of specialty publications-numeric data packages (NDPs), computer model packages (CMPs), technical reports, public communication publications, newsletters, article reprints, and reference books-in response to requests for information related to global environmental issues, primarily those pertaining to climate change. CDIAC's staff also provides technical responses to specific inquiries related to carbon dioxide $\left(\mathrm{CO}_{2}\right)$, other trace gases, and climate. Hundreds of referrals to other researchers, policy analysts, information specialists, or organizations are also facilitated by CDIAC's staff.
\end{abstract}

This report provides an account of the activities accomplished by CDIAC during the period October 1 , 1991 to September 30, 1992. An organizational overview of CDIAC and its staff is supplemented by a detailed description of inquiries received and CDIAC's response to those inquiries. An analysis and description of the preparation and distribution of numeric data packages, computer model packages, technical reports, newsletters, fact sheets, specialty publications, and reprints is provided. Comments and descriptions of CDIAC's information management systems, professional networking, and special bilateral agreements are also described.

Keywords: air pollution, ambient temperature, atmospheric chemistry, carbon cycle, carbon dioxide, climate, climate change, data analysis, data exchange, data management, earth atmosphere, emissions, fossil fuels, environmental effects, geophysical surveys, global aspects, global warming, greenhouse effect, information analysis, information management, meteorology, methane, monitoring, regional analysis, temperature monitoring, temperature surveys, trace gases 


\section{Introduction}

The Carbon Dioxide Information Center (CDIAC) was established by the U.S. Department of Energy (DOE) in 1982 to support its Carbon Dioxide Research Program, now the Global Change Research Program (GCRP). GCRP's role within DOE has been to study how atmospheric concentrations of carbon dioxide change in response to fossil-fuel emissions and o har sources of $\mathrm{CO}_{2}$ and what the response of the Earth's climate system might be. Initially, CDIAC's mission was to provide identification, collection, quality-assurance, documentation, and distribution for information on the biogeochemistry of carbon dioxide and the effects of $\mathrm{CO}_{2}$ on the earth's climate. As this research area matured, so did the scope of CDIAC, to include related global-change topics (e.g., other greenhouse gases and the effects of climate change on the environment). The flow of information into and out of CDIAC-numeric data, journal articles, agency reports, bibliographic data-has paralleled the increasing interest in the "greenhouse" issue.

Such a broad research program, involving many scientists not only in the United States but around the world, could only succeed if there was an explicit recognition of the value of information, in all its forms, and if measures were taken to ensure that this information would be freely exchanged. CDIAC's mission goes beyond supporting the research community. The education community-teachers and professors from elementary schools to university graduate departments, as well as their students-also has to be involved in this information exchange, if tomorrow's scientists are to be prepared to work on this long-term issue.

This report summarizes CDIAC's activities in collecting and distributing information during FY 1992, its eleventh year of existence.

For more information about CDIAC or to request information products from CDIAC, contact the Carbon Dioxide Information Analysis Center, Oak Ridge National Laboratory, Oak Ridge, TN 37831-6335 U.S.A.; telephone 615-574-0390, FAX 615-574-2232; TELEX 854478; electronic mail CDP@ORNLSTC (Bitnet), CDP@ORNL.GOV (Internet), or CDIAC (Omnet). 


\section{Highlights}

\section{- CDIAC Bulks Up}

Sonja Jones and Fred Stoss investigated the feasibility of using bulk mail rates for the domestic distribution of large CDIAC mailings.

This was initiated with the Winter 1992 issue of CDLAC

Communications. The estimated savings for the three major mailings is as follows:

U.S. Postage PAID

BULK RATE

Oak Ridge, Tenn.

Permit No. 3

\section{Number to \\ be sent}

4,419

4,708

4,419

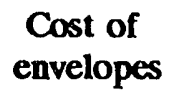

$\$ 315$

960

315

1st class
rate

$\$ 5,347$

11,111

2,298

\section{Bulk}

rate

$\$ 1,139$

2,041

1,030
Total saved

CDLAC Survey

As a result of this decision, CDIAC has saved over $\$ 16,000$ direct costs in postage charges (labor and overhead savings are not reflected here), and will save about $\$ 9,200$ more in the domestic distribution of the next two issues of CDLAC Communications. Future plans to automate the ORNL Mail Room capabilities will further reduce CDIAC's total cost for distributing its large-order offerings. The major design feature needed to facilitate bulk mailing was to create a "back cover" that has the required postage information as part of its design.

\section{- Numeric Data Packages}

During FY 1992, CDIAC issued several important new numeric data packages (NDPs) including the following:

Atmospheric $\mathrm{CO}_{2}$ Concentrations Derived from Flask Samples Collected at U.S.S.R.-Operated Sites (NDP033), based on contributions by A. M. Brounshtein, E. V. Faber, and A. A. Shashkov of the Main Geophysical Observatory in St. Petersburg, Russia, and compiled by Thomas A. Boden of CDIAC, presents data on atmospheric concentrations of carbon dioxide measured at four sites-Teriberka Station, Kotelny Island, Bering Island, and Ocean Station Charlie. This is the first numeric data package to result from data exchanges with scientists in the former Soviet Union under the auspices of Working Group VIII of the Joint Committee on Cooperation in the Field of Environmental Protection. The availability of this data set greatly enhances the global coverage for the most important greenhouse gas, providing data important in quantifying global concentrations of atmospheric carbon dioxide and in improving our understanding of the global carbon cycle.

Carbon Dioxide Enrichment: Data on the Response of Cotton to Varying $\mathrm{CO}_{2}$, Irrigation, and Nitrogen (ORNL/CDIAC-44, NDP-037) was compiled by CDIAC's Robert J. Sepanski in collaboration with the U.S. Department of Agriculture and the University of Arizona. NDP-037 presents results from field $\mathrm{CO}_{2}$-enrichment experiments conducted over five consecutive growing seasons, 1983-1987. Unlike many earlier $\mathrm{CO}_{2}$-enrichment experiments in growth chambers or greenhouses, these studies were conducted on field-planted cotton at close-to-natural conditions. The data documented in NDP-037 will be valuable for elucidating the possible future effects of an increase in atmospheric $\mathrm{CO}_{2}$ levels on an important U.S. crop. 
Two Long-Term Instrumental Climatic Data Bases of the People's Republic of China (NDP-039) was compiled by CDIAC's Dale Kaiser in collaboration with researchers at the Institute of Atmospheric Physics, Beijing. This is the first numeric data package resulting from CDIAC's participation in the U.S. DOE-PRC Joint Research on the Greenhouse Effect. NDP-039 contains monthly climate data that provide good coverage of the PRC, with the records for some stations beginning before 1900 .

United States Historical Climatology Network Daily Temperature and Precipitation Data (ORNL/CDIAC50, NDP-042) was compiled by CDIAC's Dale P. Kaiser in collaboration with researchers at the National Climatic Data Center. NDP-042 includes data from a 138-station network that was chosen from the 1219-station U.S. Historical Climatology Network. This subset was selected to provide thorough spatial coverage of the contiguous United States and a long-term record (at least 80 years for most stations) with minimal contamination from changes in instrument types/positions, time of observation, and surroundings. Thus, the data in NDP-042 are especially appropriate for use in the analysis of long-term climatic changes.

A Coastal Hazards Data Base for the U.S. East Coast NDP-043, produced by CDIAC's Tammy W. White and Richard C. Daniels in collaboration with Lamont-Doherty Geological Observatory's (LDGO) Vivien M. Gornitz, contains data for seven variables quantifying erodibility and ocean energy, presented both as standard numeric files and as GIS (geographic information system) files. As an example of possible applications of the data, the NDP presents several different coastal vulnerability indices that combine the seven variables to quantify relative risks along the coast. The NDP also includes reprints of two papers, published by Gornitz and CDIAC staff, which make use of the data to map coastal vulnerabilities along the East Coast.

\section{- CDIAC hosts visit of IGBP Database Committee}

CDIAC hosted a visit to ORNL by the Database Interface Committee, which was organized on behalf of the International Geosphere-Biosphere Programme (IGBP) by the National Research Council in cooperation with the Committee on Data for Science and Technology (CODATA). The IGBP Database Interface Committee is charged with recommending approaches to IGBP for integrating data bases from the geophysical and biological sciences. The committee's recommendations will be based in part on its discussions with CDIAC staff concerning data base acquisition, quality assurance, documentation, and distribution.

\section{- ORNL Central Research Library (CRL) Becomes Regional Information Centre}

CDIAC, in collaboration with the Oak Ridge National Laboratory (ORNL) Central Research Library (CRL), has successfully proposed to the International Council of Scientific Unions (ICSU) that the CRL be designated a Regional Information Centre for ICSU's International Geosphere-Biosphere Programme (IGBP): A Study of Global Change. The responsibilities of the IGBP Regional Information Centre will include maintaining an inventory, announcing the availability of IGBP reports, and distributing those reports on requests. The Centre will also be open for consultation to global change researchers and students. The IGBP Regional Information Centre will complement the services provided by CDIAC in its support of the DOE Global Change Research Program by increasing the accessibility of IGBP information. CDIAC was pleased to take the initiative and serve as a catalyst for this designation. 


\section{- CDIAC produces a CD-ROM}

CDIAC released a CD-ROM containing 434 megabytes of global change data from 38 numeric data packages (NDPs) and 1 computer model package (CMP).

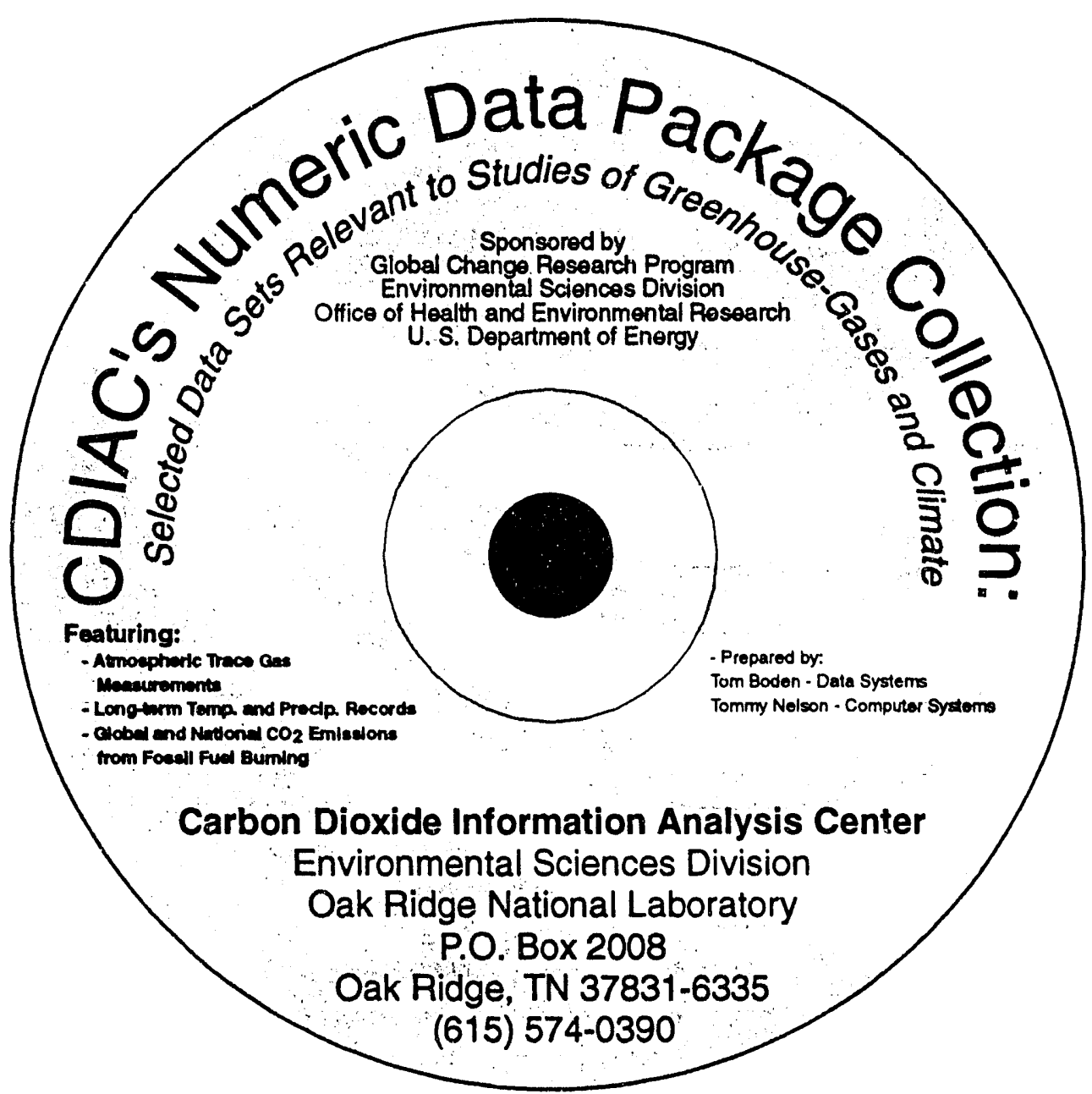

Fig. 1. CDIAC produced 200 copies of its first CD-ROM, which stores the equivalent of about 217,000 pages of data.

\section{- Numeric Files on FTP File}

CDIAC established an anonymous file transfer protocol (FTP), making CDIAC's numeric data files more readily available to customers with Internet access. Automated routines electronically send FTP users a brief description of CDIAC, the services we offer, what numeric data sets are provided, and how to access them. A total of 145 FTP transfers were processed by CDIAC in FY 1992. 


\section{Highlights from CDIAC's Related Activities}

\section{- Global Historical Climatology Network (GHCN)}

The Carbon Dioxide Information Analysis Center (thanks to separate funding from the DOE Giobal Change Research Program's Quantitative Links program) and the National Climatic Data Center have initiated the Global Historical Climatology Network (GHCN) project. Its purpose is to compile an improved global climate data base of monthly mean temperature, precipitation, sea level pressure, and station pressure for thousands of meteorological stations worldwide. Specifically, the GHCN seeks to acquire numerous (preexisting) national- regional- and global-scale data sets; to scrutinize and upgrade the quality of each data set; and to consolidate them into a single global climate data base. This data base has been issued by CDIAC as NDP-041.

The Global Historical Climatology Network: Long-Term Monthly Temperature, Precipitation, Sea Level Pressure, and Station Pressure Data (ORNL/CDIAC-53, NDP-041) was compiled by CDIAC's Russell S. Vose in collaboration with P. M. Steurer, T. C. Peterson, R. Heim, and T. R. Karl of the National Oceanic and Atmospheric Administration, J. K. Eischeid of Cooperative Institute for Research in Environmental Sciences (CIRES), and Richard L. Schmoyer of the Oak Ridge National Laboratory. NDP-041 provides a quality-assured data base with global coverage (more than 6000 stations for temperature and more than 7500 stations for precipitation) and a long-term record (40\% of the stations have more than 50 years of data). It represents the best available data base for studies of global climate change.

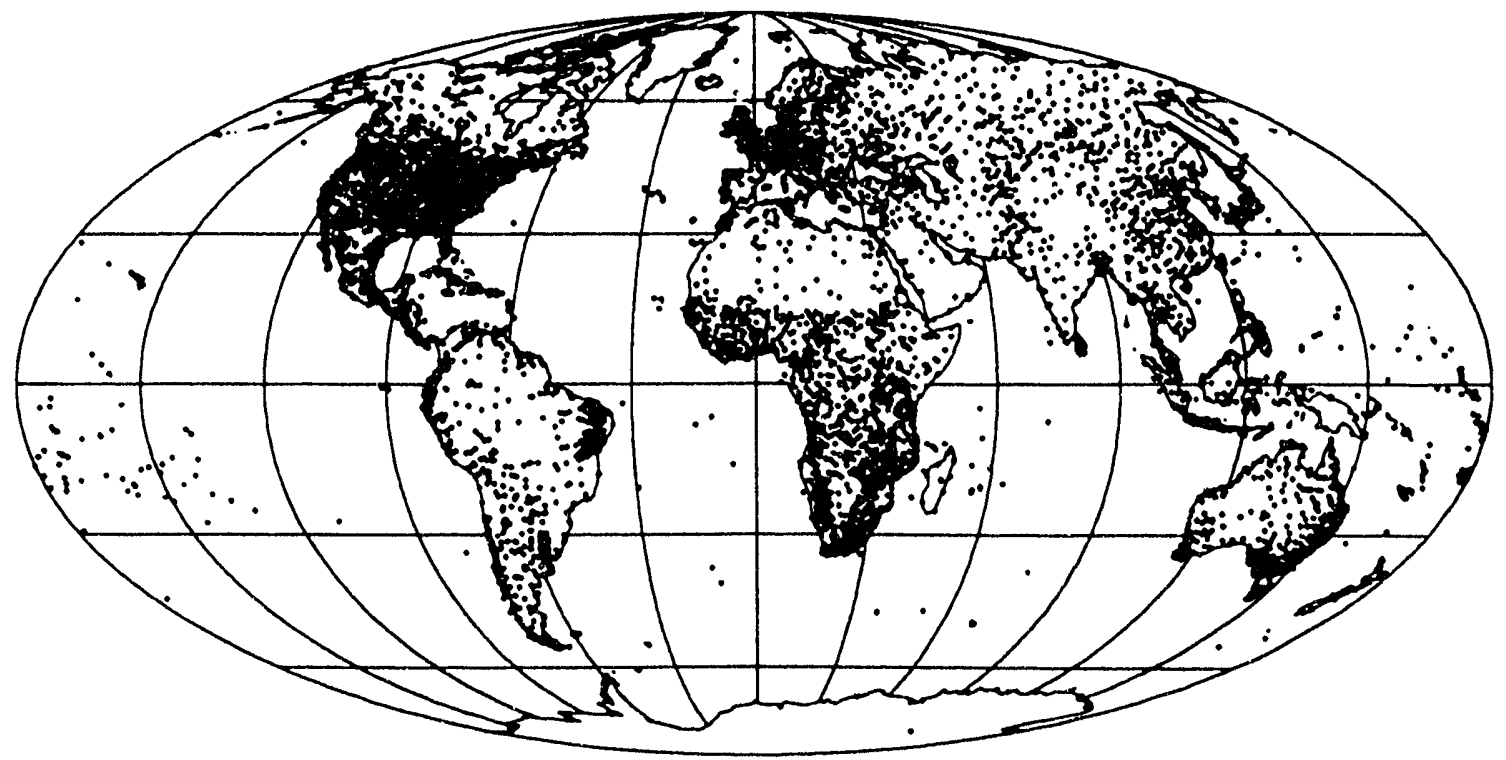

Fig. 2. Locations of the more than 7500 precipitation stations contained in the Global Historical Climatology Network data base. 


\section{- ARM Outreach}

The Carbon Dioxide Information Analysis Center (CDIAC) has produced the first issue of the new newsletter, ARM Outreach, based on separate funding from the DOE Global Change Research Program's Atmospheric Radiation Measurement (ARM) Program (front cover pictured below). The purpose of $A R M$ Outreach is to inform a broad audience of researchers, policymakers, managers, and educators about ARM's research and program activities. Volume 1, No. 1 of $A R M$ Outreach describes the ARM Program's setting within DOE; its purposes, goals, and organization; and its investigators and their research. This inaugural issue also presents summaries of two research projects conducted under the aegis of the ARM Program.

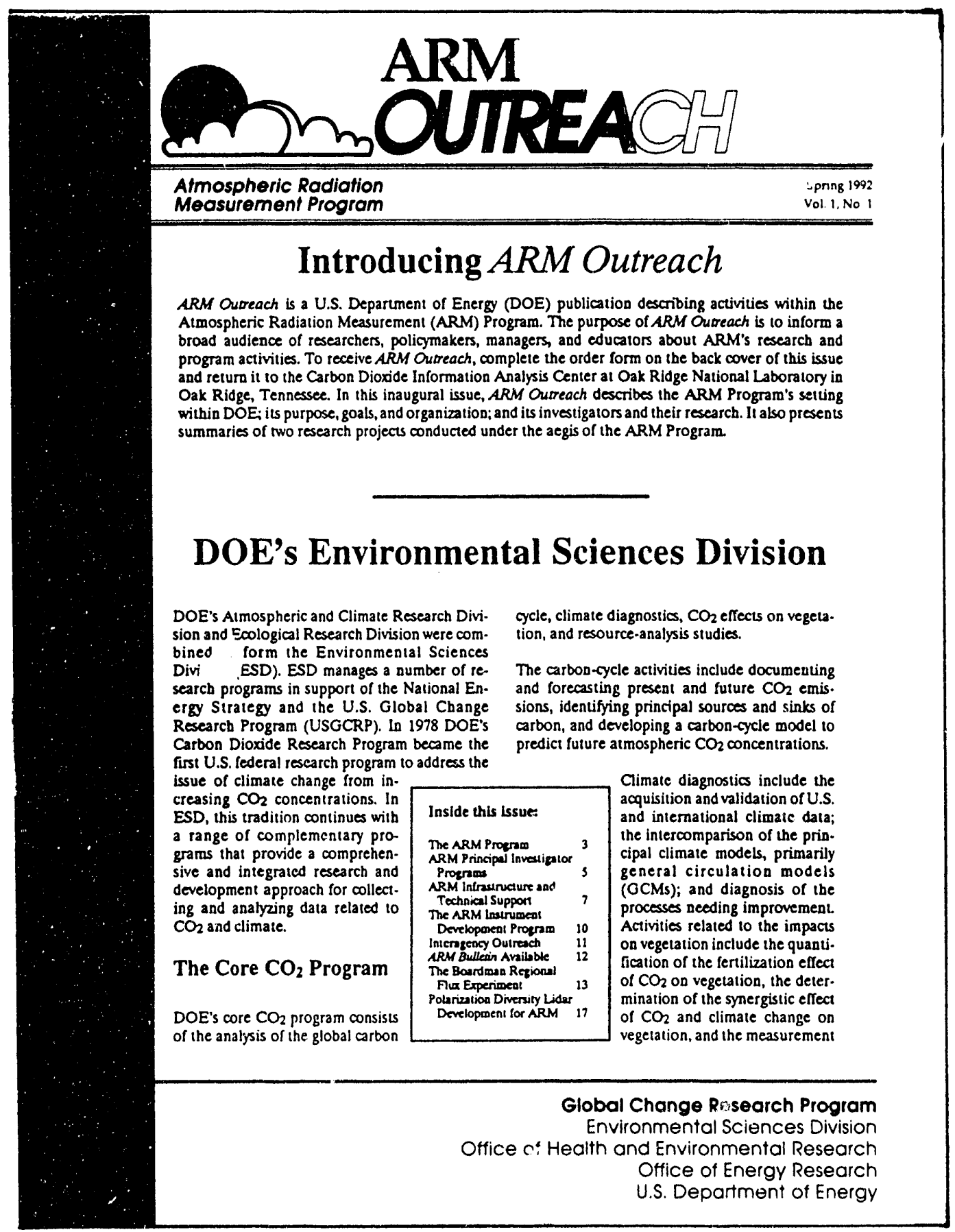


- Russian-English Translation Project

CDIAC, in collaboration with the Research Institute of Hydrometeorological Information-World Data Center in Obninsk, has begun to produce a series of technical reports based on the translation into English of foreign-language literature, with separate funding from the DOE GCRP's Quantitative Links program. This task includes the evaluation of translation software developed for the personal computer.

Many important climate-related manuscripts, reports, and books have been published in the Russian language only, thereby limiting their usefulness to the global climate-change community. The task began with the identification of important Russian-language literature in the four areas identified as being of particular importance in the Quantitative Links data survey-surface energy budget, aerosols, clouds, and general circulation models.

Next a technical translator was brought to CDIAC to translate the abstracts. As part of this process, personal-computer-based software for Russian-to-English translation was evaluated.

Finally, the first dual-language document in this series, Selected Translated Abstracts of RussianLanguage Climate-Change Publications: I. Surface Energy Budget, was produced as a joint CDIAC-RIHMI publication (Ravina, C. B., and M. D. Burtis. 1992. ORNL/CDIAC-57; Proceedings of RIHMI-WDC, Number 158. Oak Ridge National Laboratory, Oak Ridge, Tenn.), with appropriate credit given to both collaborating organizations. This task was conducted under the auspices of Working Group VIII of the U.S.U.S.S.R. bilateral agreement, which has included joint publication as one of its activities.

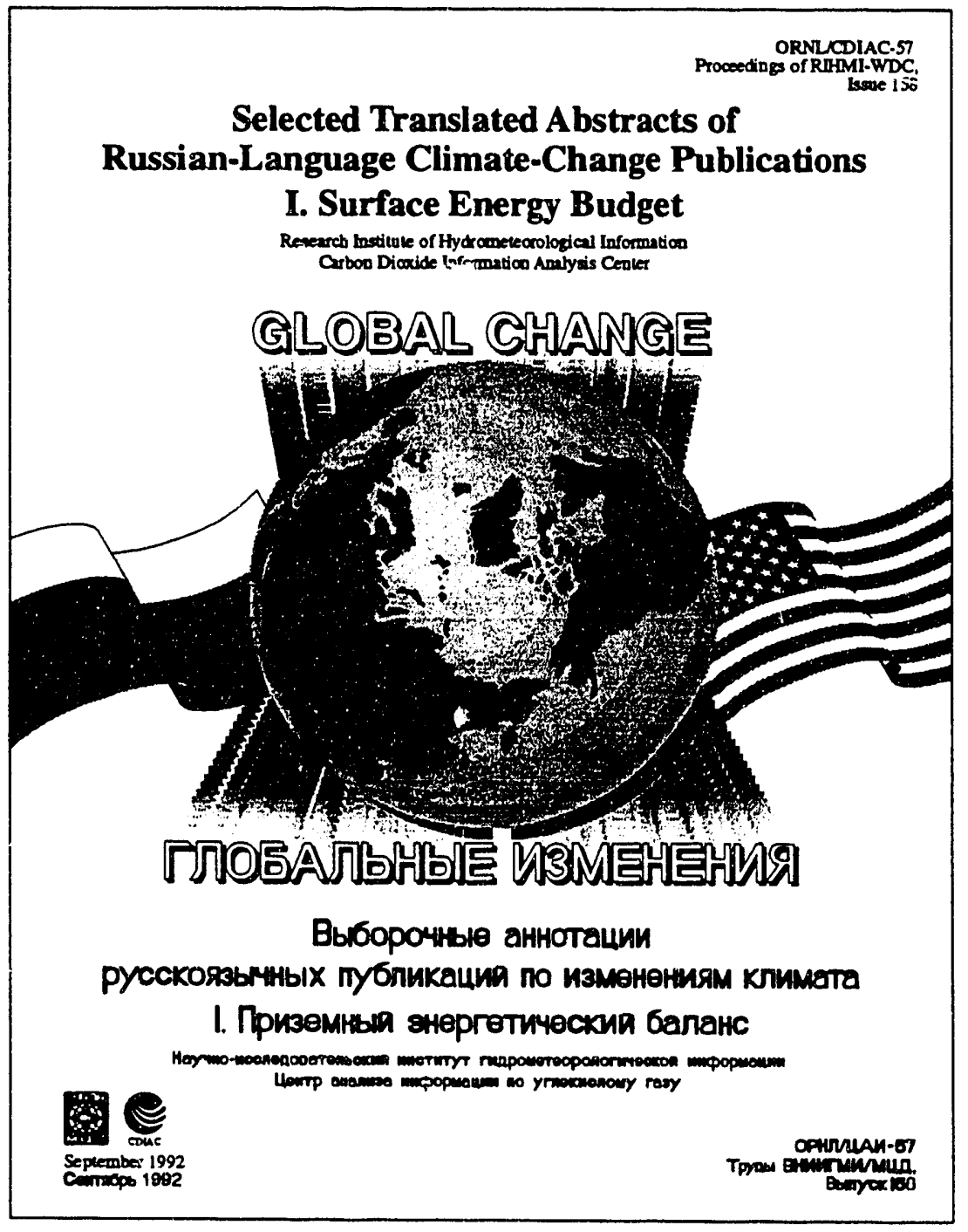

Fig. 3. The first dual-language document in the series, Selectcd Translated Abstracts of Russian-Language ClimateChange Publications: I. Surface Energy Budget. 


\section{Organization and Staff}

Bob Cushman replaced Paul Kanciruk as the Director of CDIAC and Patty Crabtree is CDIAC's secretary. CDIAC reports programmatically to the Environmental Information Analysis Program (managed by Paul Kanciruk) and CDIAC staff report administratively to the Environmental Analyses Section (headed by Frances E. Sharples) both in the Environmental Sciences Division (ESD) at ORNL. CDIAC consists of three groups (Fig. 4): Data Systems, Information Systems, and Computer Systems, headed, respectively, by Thomas A. Boden, Frederick W. Stoss, and Tommy R. Nelson. The Data Systems group handles the quality assurance and documentation of data which is the cornerstone of CDIAC. Through its Information Systems group, CDIAC provides a forum for the exchange of data among researchers and is active in a network of information organizations and special libraries and in publicizing its products and services. CDIAC's Computer Systems group, in addition to maintaining CDIAC's own network of personal computers, work stations, and links with ORN''s mainframe computers, strives to ensure that CDIAC's system is compatible with that of its user community. Table 1 lists the CDLAC staff and their titles.

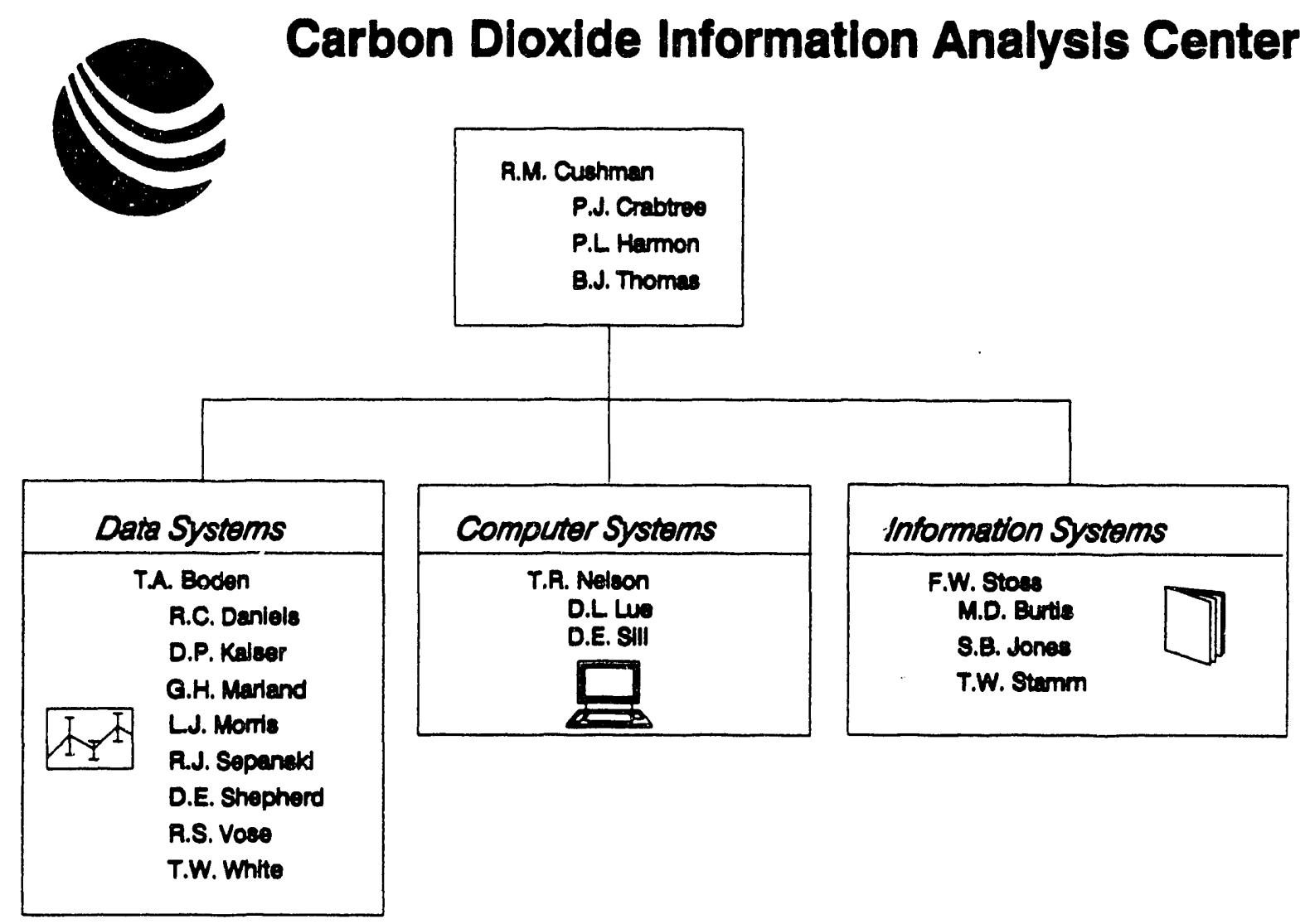

Fig. 4. CDIAC organization (September 30, 1992). 
Table 1. CDIAC staff, FY 1992

\begin{tabular}{ll}
\hline \multicolumn{1}{c}{ Staff } & \multicolumn{1}{c}{ Title } \\
\hline Thomas A. Boden & Task Leader, Data Systems \\
Marvel D. Burtis & Editorial Assistant \\
Patricia J. Crabtree & Secretary \\
Robert M. Cushman & Director, CDIAC \\
Richard C. Daniels* & Data Packaging Specialist \\
Penny L. Harmon & Secretary/Telephone Receptionist \\
Sonja B. Jones* & Request-Response Coordinator \\
Dale P. Kaiser* & Data Packaging Specialist \\
Don L. Lue* & Personal Computing Specialist \\
Gregg Marland & Senior Scientist \\
Laura J. Morris & Data Packaging Assistant \\
Tommy R. Nelson** & Task Leader, Computer Systems \\
Robert J. Sepanski* & Data Packaging Specialist \\
Deborah E. Shepherd & Data Packaging Assistant \\
David E. Sill** & Workstation Specialist \\
Timothy W. Stamm* & Request-Response Associate \\
Frederick W. Stoss* & Task Leader, Information Systems \\
B. Jeanne Thomas & Office Assistant \\
Russell S. Vose* & Data Packaging Specialist \\
Tammy W. White & Geographic Information Systems Specialist \\
\hline
\end{tabular}

*Energy, Environment, and Resources Center, University of Tennessee, Knoxville

${ }^{* *}$ Computing and Telecommunications Division, ORNL 


\section{Professional Development}

During the year CDIAC staff are encouraged to attend various workshops and training programs. These opportunities allow staff to gain new skills and improve their expertise in areas related to CDIAC's operations. The following is a list of CDIAC staff professional development activities for FY 1992.

\section{Training}

- Dale Kaiser completed the SAS Ma Macro Language training course in Cary, NC, March 24-25, 1992.

- Tom Boden completed a SAS'm PC Graphics course in Irvine, California (October 22-26, 1991).

- Fred Stoss attended the Special Libraries Association Middle Management Institute, taking a 21/2-day continuing education course, Information Management: Technologies and Applications.

- Fred Stoss attended a 1-day training course, Managing Multiple Projects, Objectives, and Deadlines.

- Debbie Shepherd completed the SAS ${ }^{M}$ Fundamentals course, at the Oak Ridge Y-12 site, Oct.-Nov. 1991.

- Debbie Shepherd completed the Basics of DOS course, in Knoxville, March 1992.

\section{Publications and Presentations}

- Russ Vose was lead author on an article entitled "The Global Historical Climatology Network: Long-Term Monthly Temperature, Precipitation, Sea Level Pressure, and Station Pressure Data." The paper will be published in January 1993 in the Proceedings of the Fourth Symposium on Global Change Studies, sponsored by the American Meteorological Society.

- An abstract submitted by Fred Stoss entitled, "The Carbon Dioxide Information Analysis Center: Providing Support for DOE's Global Change Research Program," was accepted for presentation at the DOE OSTI 1992 INFOTECH Conference, which was held in Oak Ridge, Tennessee.

- Bob Cushman's and Fred Stoss' manuscript, "The Carbon Dioxide Information Analysis Center," was accepted and revised for publication in the journal, Environment, as one of the journal's institutional profiles. This general interest piece describes the history of CDIAC and reviews CDIAC's services, products, and publications.

- Russ Vose presented a paper entitled "Compilation of Long-Term Monthly Temperature, Precipitation, and Pressure Data Sets" at the annual Association of American Geographers Meeting in San Diego, California in April 1992.

- Tammy White presented "What is a GIS" to the Environmental Sciences Division; Martin Marietta Energy Systems, Inc. Self-Directed Work Team Task Force; and Clyde Hopkins, President of Martin Marietta Energy Systems, Inc.

- Cloud Amount and Sunshine Duration in the People's Republic of China, 1954-88 (Paper accepted for presentation by Dale Kaiser at the 8th Conference on Applied Climatology, January 1993 at Anaheim, California. The paper will appear in the preprint volume of the conference.) Average conditions and trends in cloud amount and sunshine duration over the period 1954-88 are analyzed, the data being obtained from NDP-039, Two Long-Term Instrumental Climatic Data Bases of the People's Republic of Cnina, which was completed by Dale in FY 1991. 


\section{Committees and Appointments}

- Paul Kanciruk continued to serve as a member of DOE Interagency Working Group on Data Management for Global Change.

- Fred Stoss was named a member of the Library/Information Subgroup (LIS) of the Interagency Working Group on Data Management for Global Change.

- Fred Stoss vas named a member of the International Board of Consulting Editors for the recently founded Green Library Journal, and asked to continue to serve as a Technical Advisor to R. R. Bowker's Environment Abstracts.

- Fred Stoss was named as a member of the Editorial Board of the Green Library Journal: Environmental Topics in the Information World.

- Fred Stoss was selected to serve on the Committee to Review the Election Process for the Special Libraries Association. This committee was authorized by SLA's Board of Directors and appointed by its President, Guy St. Clair to review the process of electing national officers and directors.

- Gregg Marland served as a member of the Global Climate Task Force of the Council for Agricultural Science and Technology.

- Fred Stoss was asked to consider a part-time detail (six months over a one year period) to assist the Council on Environmental Quality with activities related to the Interagency Committee on Environmental Trends.

- Rich Daniels and Tammy White serve as members of the Environmental Sciences Divisions (ESD) add-hoc GIS Committee. The Committee has been responsible for fostering the development of a self supported GIS work team and the design and implementation of a GIS upgrade path in the division.

- Tammy White served as Co-chairperson for ESD Self Directed Work Team and for ESD GIS Operations Committee, FY 1992, and had a leadership role in the development of FY 1992 and FY 1993 operations plans for ESD GIS.

- Rich Daniels was a co-team leader during ORNL-1992 Science Honors Workshop. The team worked with four high school students over a two-week period in July.

- Rich Daniels volunteered to coordinate printer/terminal/digitizer interconnections for the Environmental Sciences Division's GIS Users Team (October 1991 to present).

\section{Awards and Honors}

- CDIAC received an Award of Achievement from the East Tennessee Chapter of the Society for Technical Communications for its production of CDLAC Communications. This is the publication's 5th ETC/STC award. 


\section{Request-Response Records $\left(\mathbf{R}^{3}\right)$ System}

When CDIAC began in 1982, greenhouse warming was a topic still primarily in the research arena and CDIAC's role was, for the most part, one of distributing data from researchers to researchers. By 1990 , however, when greenhouse warming was front-page news, the subject of newsmagazine lead stories, congressional attention, and international conferences; CDIAC's role-and its user community-had broadened considerably. CDIAC has been fielding information requests from congressional staffers drafting or evaluating legislation, from public-school students working on science fair projects, and from science reporters compiling data for stories, as well as from research scientists. Table 2 is a summary of CDIAC request/response activities for FY 1992.

Table 2. Request/response activities

\begin{tabular}{|c|c|c|c|c|}
\hline \multirow[b]{3}{*}{ Category } & \multicolumn{4}{|c|}{ Number of requests } \\
\hline & \multicolumn{3}{|c|}{ Fiscal Year 1992} & \multirow{2}{*}{$\begin{array}{l}\text { Total } \\
\text { FY 1985 } \\
\text { to date }\end{array}$} \\
\hline & Qtrs 1-2 & Qtrs 3-4 & Total & \\
\hline$\overline{\text { Discussions }^{2}}$ & 1,501 & 1,825 & 3,326 & 16,777 \\
\hline DOE reports & 451 & 241 & 692 & 5,420 \\
\hline CDIAC reports & 890 & $1, .272$ & 2,162 & 6,476 \\
\hline Other reports & 182 & 86 & 268 & 825 \\
\hline Data processing & 15 & 0 & 15 & 282 \\
\hline Articles & 22 & 30 & 52 & 451 \\
\hline $\mathrm{NDPs}^{\mathrm{b}}$ & 214 & 478 & 692 & 2,389 \\
\hline CMPs & 21 & 80 & 101 & 456 \\
\hline $\begin{array}{l}\text { Trends } \\
\text { (on diskette) }\end{array}$ & 8 & 109 & 117 & 117 \\
\hline Directory searches & 385 & 408 & 793 & 3,925 \\
\hline Miscellaneous & & 93 & 93 & 93 \\
\hline Networking & & 5 & 5 & 5 \\
\hline Total & 3,689 & 4,627 & 8,316 & 37,216 \\
\hline
\end{tabular}

${ }^{a}$ Discussions include telephone conversations, letters, and interoffice correspondence that pertain to DOE deliverables or other global change-related matters. It does not include day-to-day operations at CDLAC.

bRequests for CD-ROM included.

- CDIAC responded to 8,316 requests from 3,326 individuals for CDIAC products and services from 72 countries: Algeria, Argentina, Australia, Austria, Bangladesh, Barbados, Belgium, Belize, Bermuda, Botswana, Brazil, Bulgaria, Canada, Chile, Colombia, Costa Rica, Cuba, Cyprus, Czechoslovakia, Denmark, Egypt, Ethiopia, Fiji, Finland, France, Germany, Ghana, Hong Kong, Hungary, India, Indonesia, Ireland, Israel, Italy, Japan, Jordan, Kenya, Luxembourg, Malaysia, Maldives, Mexico, Monaco, Morocco, Nepal, Netherlands, New Zealand, Niger, Nigeria, North Korea, Norway, People's Republic of China, Philippines, Poland, Portugal, Romania, Russia, Saudi Arabia, Singapore, Somalia, South Africa, South Korea, Spain, Sri Lanka, Sweden, Switzerland, Taiwan, Thailand, Turkey, United Kingdom, United States, Venezuela, and Yugoslavia (Figs. 5 and 6). 
- CDIAC responded to 3 requests for the data presented in the technical report $A$ Comprehensive Precipitation Data Set for Global Land Areas (TR051, DOE/ER-69017T-H1, April 1991, 82 p.); 2 magnetic tapes and one FTP transfer were generated in filling these requests.

- A total of 37,107 requests from 100 countries have been filled from FY 1985 to date (Figs. 7, 8, and 9).

- During FY 1992 CDIAC received a total of 3,122 requests for reports (DOE, CDIAC, and others), including

DOE reports

692 requests

2,836 copies distributed

CDIAC reports

2,162 requests

10,139 copies distributed

Other reports

268 requests

316 copies distributed

Total reports distributed: 13,291 


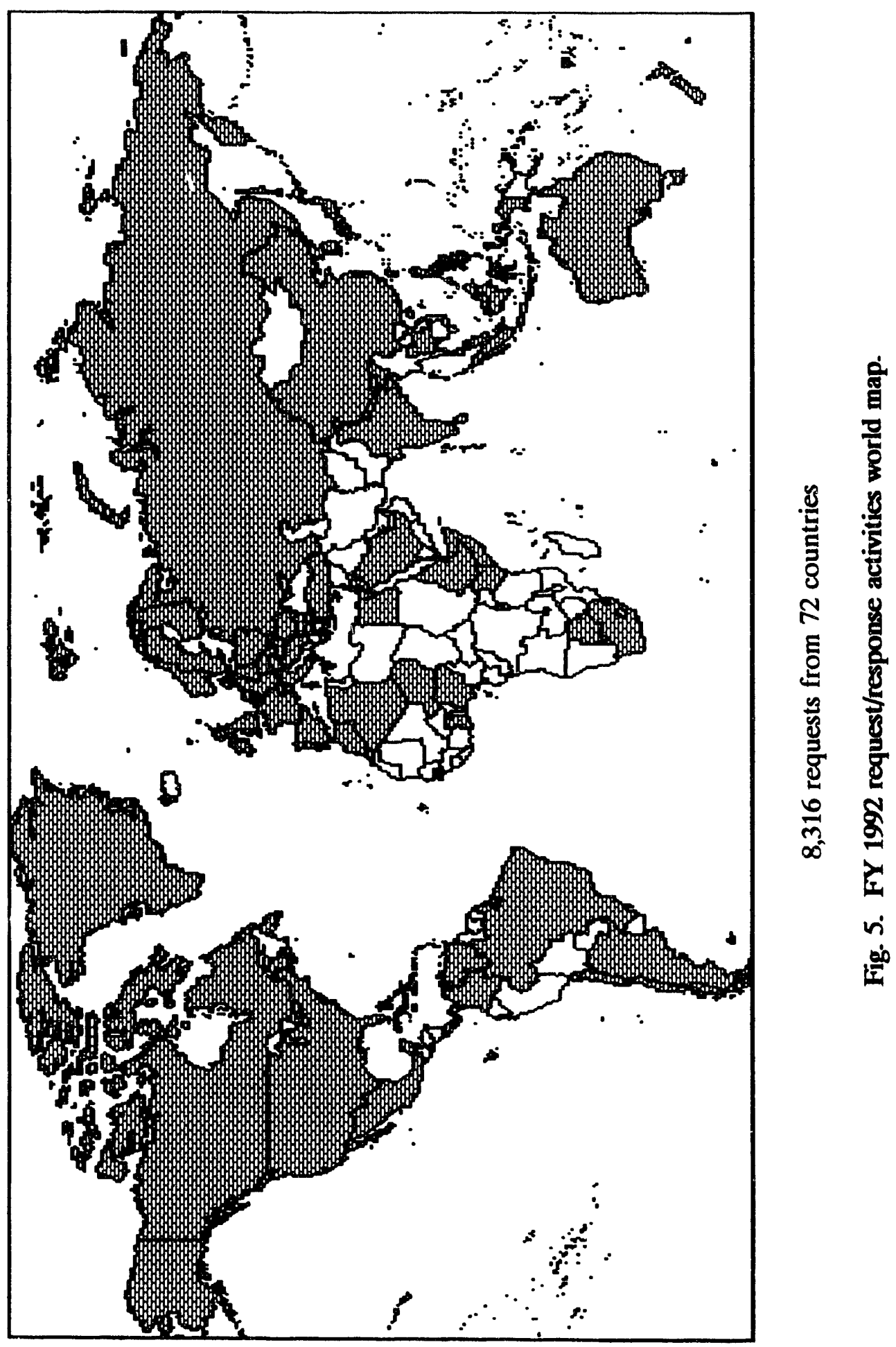




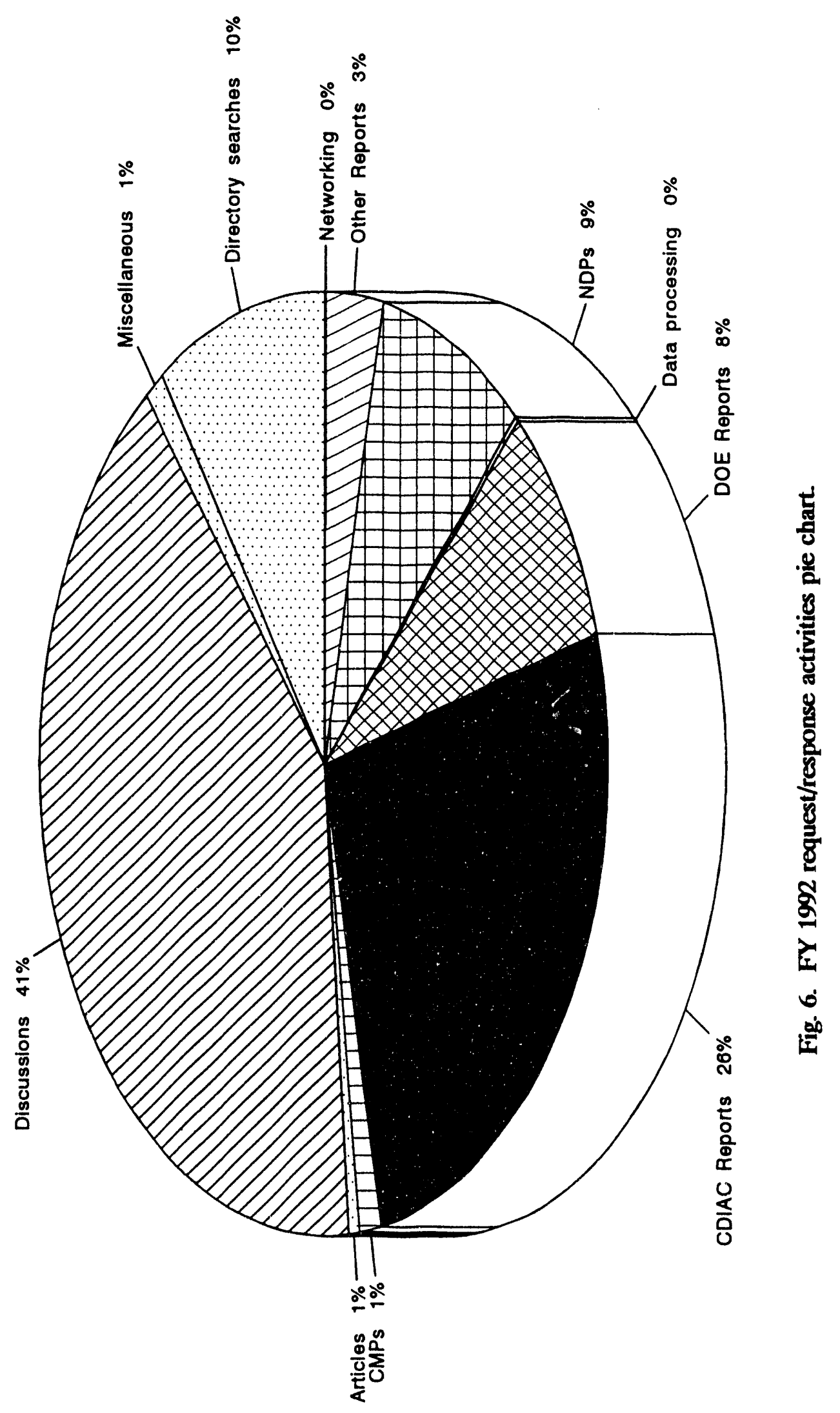




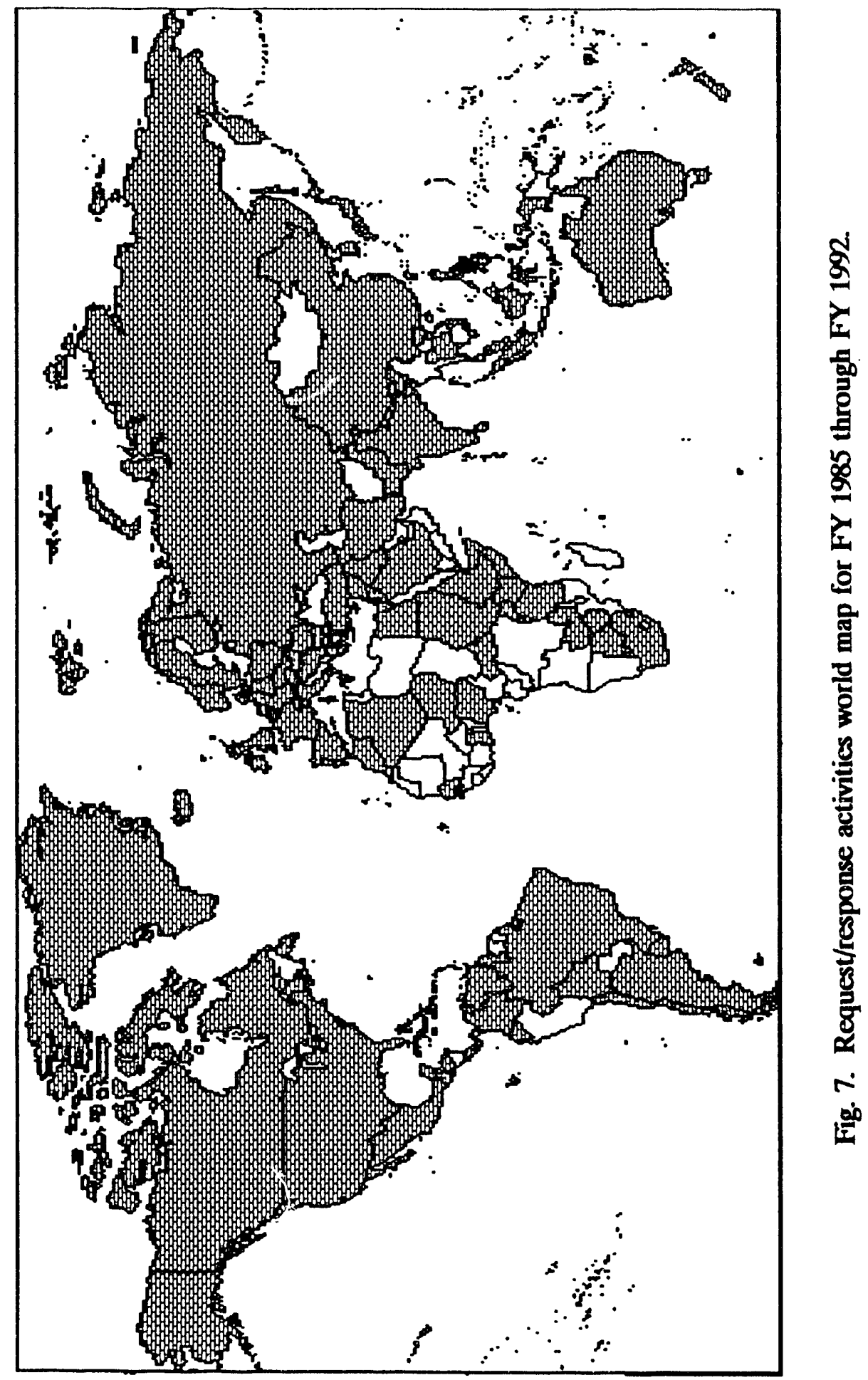




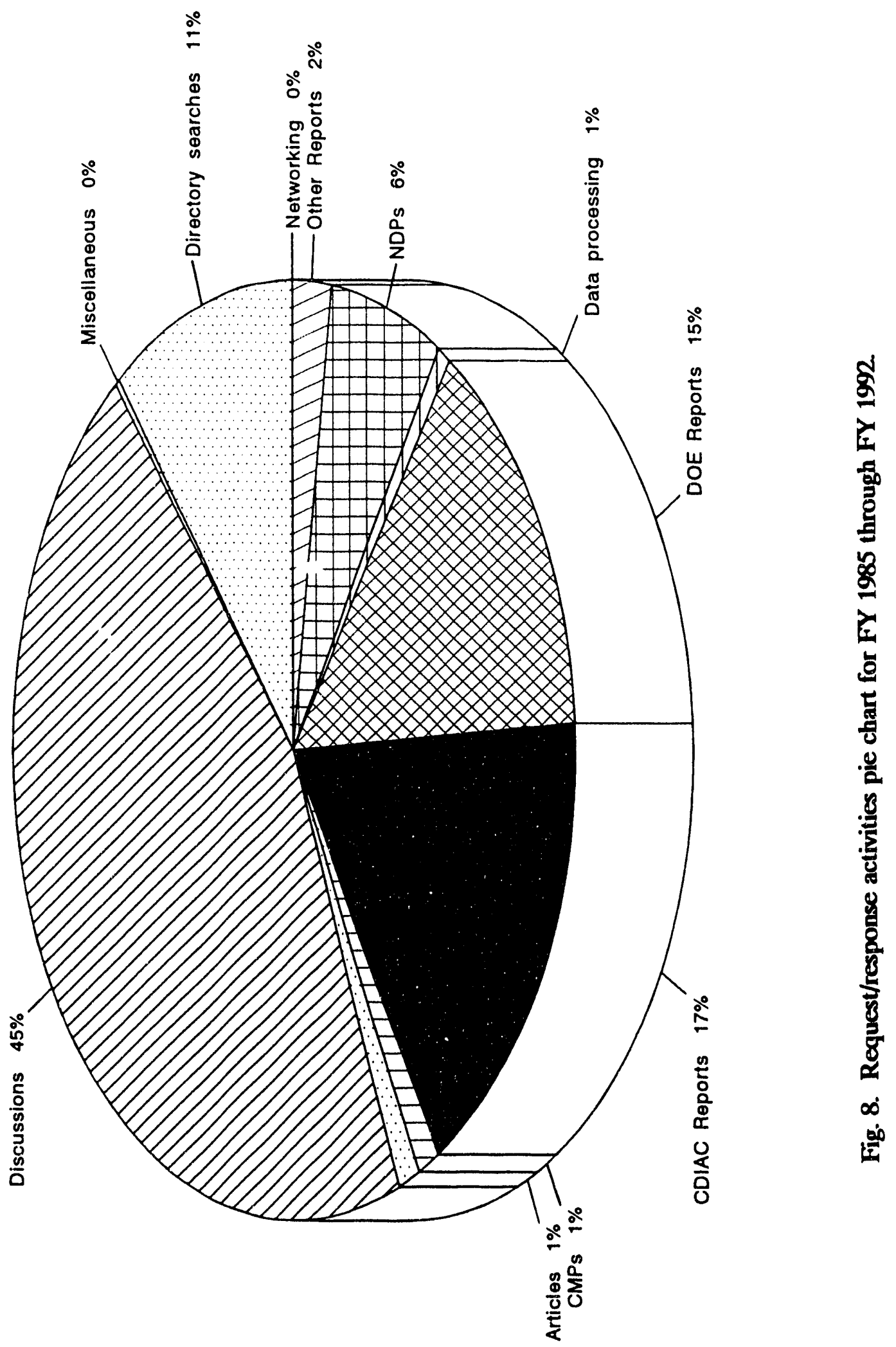




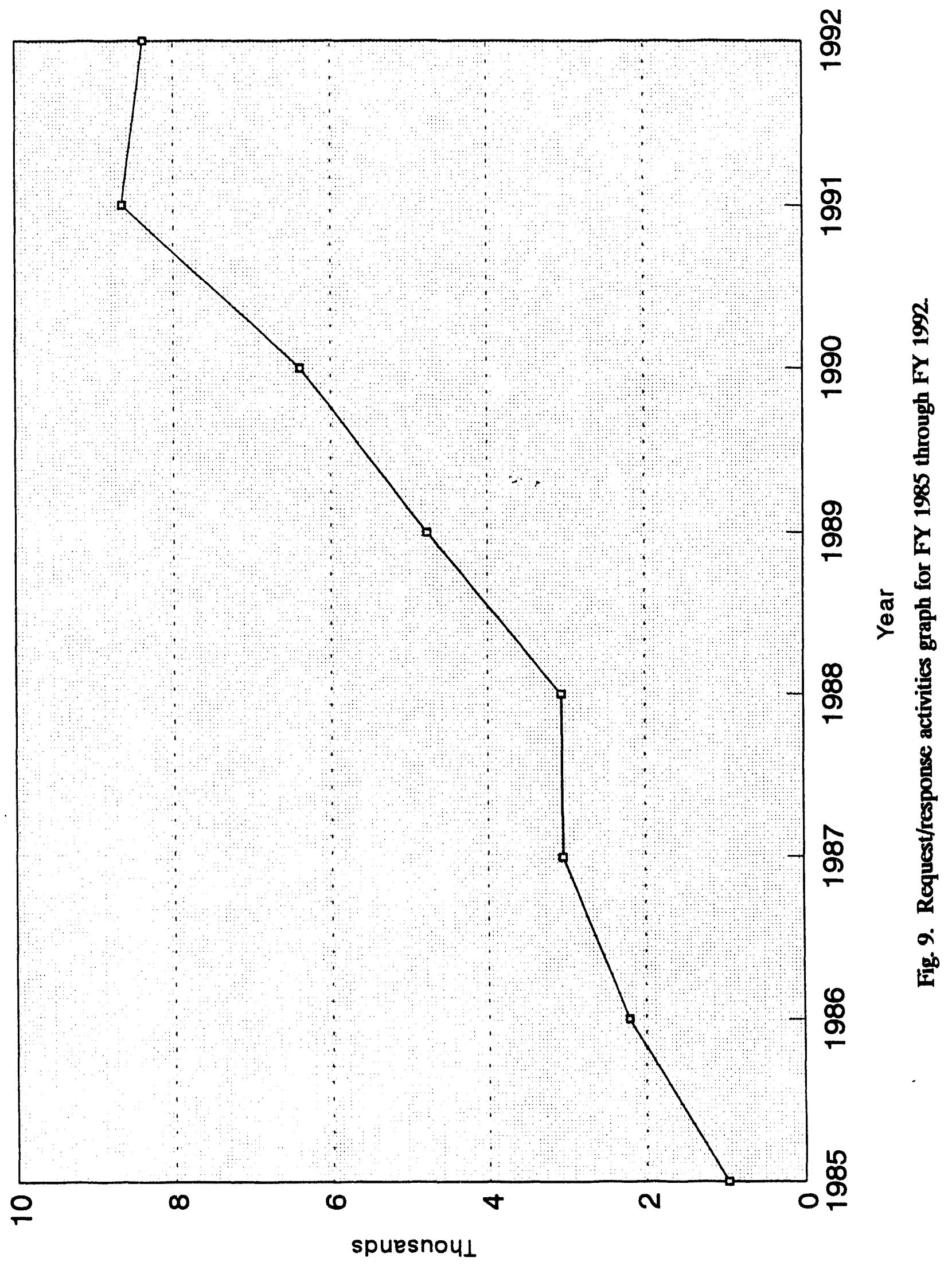


Table 3. DOE reports distribution

\begin{tabular}{|c|c|c|c|c|c|c|}
\hline \multirow{2}{*}{$\begin{array}{l}\text { Report } \\
\text { number }\end{array}$} & \multirow[b]{2}{*}{ Title and contents } & \multicolumn{2}{|c|}{ FY 1992} & \multirow[b]{2}{*}{ Total } & \multirow{2}{*}{$\begin{array}{l}\text { Total } \\
\text { FY } 1985 \\
\text { to date }\end{array}$} & \multirow{2}{*}{$\begin{array}{l}\text { Remaining } \\
\text { copies }\end{array}$} \\
\hline & & Qtrs 1-2 & Qtrs 3.4 & & & \\
\hline 001 & $\begin{array}{l}\text { Workshop on the Global } \\
\text { Effects of Carbon Dioxide } \\
\text { From Fossil Fuels, May } 1979\end{array}$ & 1 & & 1 & 26 & N/A \\
\hline 002 & $\begin{array}{l}\text { Summary of the Carbon } \\
\text { Dioxide Effects and } \\
\text { Assessment Program }\end{array}$ & & & & 12 & N/A \\
\hline 003 & $\begin{array}{l}\text { The Role of Temperate Zone } \\
\text { Forests in the World Carbon } \\
\text { Cycle, February } 1980\end{array}$ & & & & 25 & N/A \\
\hline 004 & $\begin{array}{l}\text { The Role of Organic Soils } \\
\text { in the World Carbon Cycle, } \\
\text { February } 1980\end{array}$ & & & & 25 & N/A \\
\hline 005 & $\begin{array}{l}\text { Carbon Dioxide Research } \\
\text { Progress Report, April } 1980\end{array}$ & 5 & 1 & 6 & 49 & 113 \\
\hline 006 & $\begin{array}{l}\text { Environmental Control } \\
\text { Technology for Atmospheric } \\
\text { Carbon Dioxide, May } 1980\end{array}$ & & & & 18 & 8 \\
\hline 007 & $\begin{array}{l}\text { The Role of Tropical } \\
\text { Forests in the World } \\
\text { Carbon Cycle, August } 1980\end{array}$ & & & & 58 & 5 \\
\hline 008 & $\begin{array}{l}\text { A Comprehensive Plan for } \\
\text { Effects Research and } \\
\text { Assessment, Part 1: The } \\
\text { Global Carbon Cycle and } \\
\text { Climatic Effects of In- } \\
\text { creasing Carbon Dioxide }\end{array}$ & 3 & & 3 & 44 & 71 \\
\hline 009 & $\begin{array}{l}\text { Workshop on Environmental } \\
\text { and Societal Consequences } \\
\text { of a Possible } \mathrm{CO}_{2} \text {-Induced } \\
\text { Climate Change, September } \\
1980\end{array}$ & 4 & 3 & 7 & 96 & 37 \\
\hline 010 & $\begin{array}{l}\text { Measurement of Changes in } \\
\text { Terrestrial Carbon Using } \\
\text { Remote Sensing }\end{array}$ & & & & 13 & 8 \\
\hline 011 & $\begin{array}{l}\text { Proceedings of the Carbon } \\
\text { Dioxide and Climate } \\
\text { Research Program Conference, } \\
\text { December } 1980\end{array}$ & 2 & & 2 & 50 & 26 \\
\hline
\end{tabular}


Table 3. (continued)

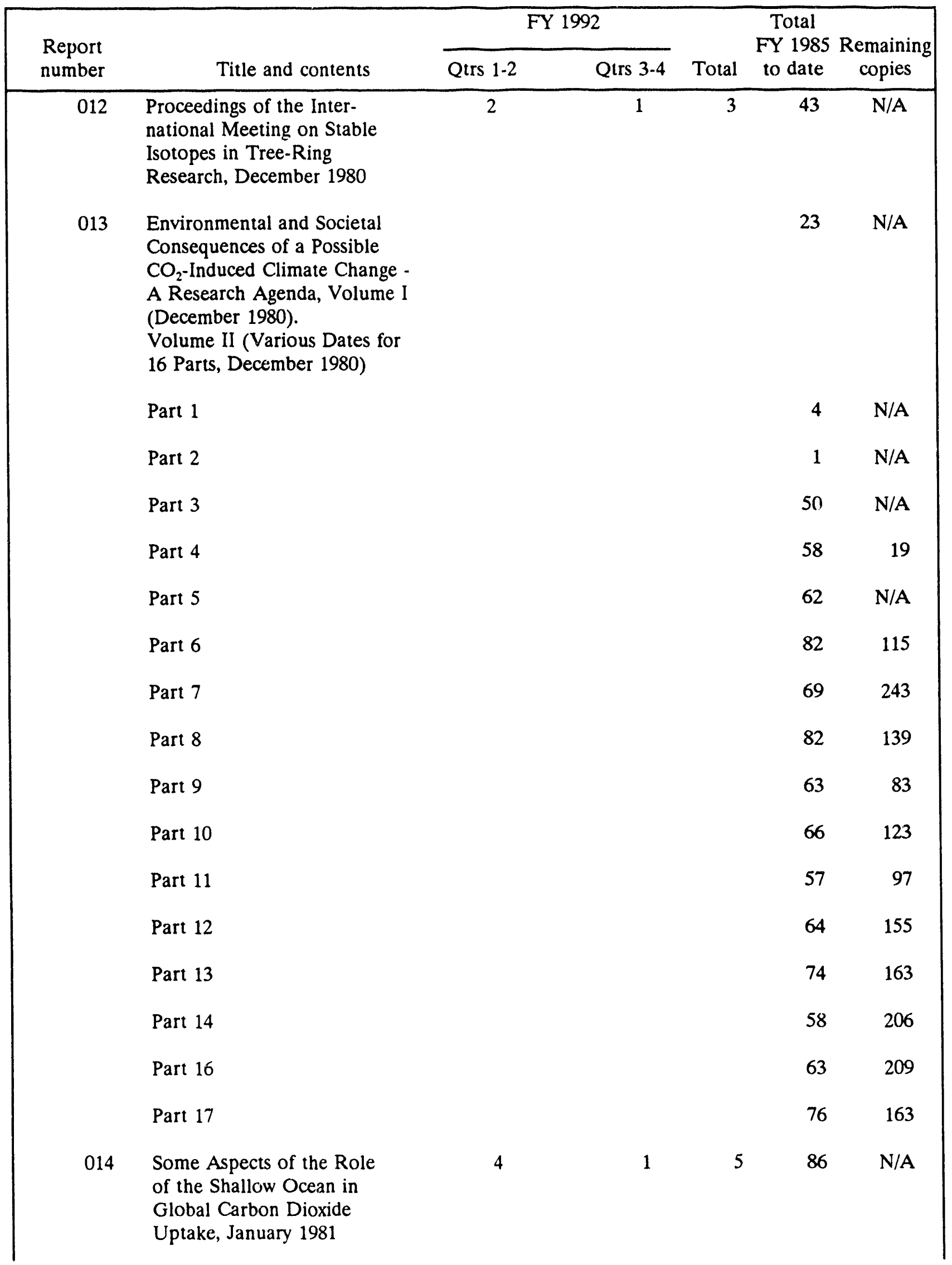


Table 3. (continued)

\begin{tabular}{|c|c|c|c|c|c|c|}
\hline \multirow{2}{*}{$\begin{array}{l}\text { Report } \\
\text { number }\end{array}$} & \multirow[b]{2}{*}{ Title and contents } & \multicolumn{2}{|c|}{ FY 1992} & \multirow[b]{2}{*}{ Total } & \multirow{2}{*}{$\begin{array}{l}\text { Total } \\
\text { FY } 1985 \\
\text { to date }\end{array}$} & \multirow{2}{*}{$\begin{array}{l}\text { Remaining } \\
\text { crspies }\end{array}$} \\
\hline & & Qtrs 1-2 & Qtrs 3-4 & & & \\
\hline 015 & $\begin{array}{l}\text { Carbon Balance in Northern } \\
\text { Ecosystems and the Potential } \\
\text { Effect of Carbon Dioxide- } \\
\text { Induced Climatic Change, } \\
\text { Jancary } 1981\end{array}$ & & & & 21 & N/A \\
\hline 016 & $\begin{array}{l}\text { Flux of Organic Carbon by } \\
\text { Rivers to the Oceans, } \\
\text { April } 1981\end{array}$ & 5 & & 5 & 55 & 52 \\
\hline 017 & $\begin{array}{l}\text { Workshop on Oceanic } \mathrm{CO}_{2} \\
\text { Standardization, February } \\
1982\end{array}$ & 2 & & 2 & 61 & 198 \\
\hline 018 & $\begin{array}{l}\text { Proceedings of the Wurkshop } \\
\text { on First Detecticu of Carbon } \\
\text { Dioxide Etfects, May } 1982\end{array}$ & 2 & 1 & 3 & 56 & N/A \\
\hline 019 & $\begin{array}{l}\text { Global Dynamics of Biospheric } \\
\text { Carbon, July } 1982\end{array}$ & & & & 15 & N/A \\
\hline 020 & $\begin{array}{l}\text { Effect of } \mathrm{CO}_{2} \text { on Man } \text { malian } \\
\text { Organisms, December } 1982\end{array}$ & & & & 19 & N/A \\
\hline 021 & $\begin{array}{l}\text { Proceedings: Carbon Dioxide } \\
\text { Research Conference: Carbon } \\
\text { Dioxide, Science and } \\
\text { Consensus, February } 1983\end{array}$ & 3 & 1 & 4 & 43 & N/A \\
\hline 022 & $\begin{array}{l}\text { Conf } 3608144 \text { - Proceedings } \\
\text { of the International } \\
\text { Symposium of Ecological } \\
\text { Aspests of Tree Ring Analysis }\end{array}$ & 5 & 5 & 10 & 57 & 29 \\
\hline 023 & $\begin{array}{l}\text { Conf } 8708252 \text { - Proccedings } \\
\text { from the 2nd Annual Science } \\
\text { Meeting of the USDOE and } \\
\text { PRCAS Joint Research Program }\end{array}$ & 2 & & 2 & 40 & N/A \\
\hline 024 & $\begin{array}{l}\text { Workshop on Sea Level Rise } \\
\text { and Coastal Processes, March } \\
1989\end{array}$ & 11 & 3 & 14 & 175 & 61 \\
\hline $\begin{array}{l}\text { CONF- } \\
890525\end{array}$ & $\begin{array}{l}\text { Proceedings of the Inter- } \\
\text { national Conference on Global } \\
\text { and Regional Environmental } \\
\text { Atmospheric Chemistry, } \\
\text { Beijing, China, May 3-10, } \\
1989\end{array}$ & & 3 & 3 & 46 & N/A \\
\hline
\end{tabular}


Table 3. (continued)

\begin{tabular}{|c|c|c|c|c|c|c|}
\hline \multirow{2}{*}{$\begin{array}{l}\text { Report } \\
\text { number }\end{array}$} & \multirow[b]{2}{*}{ Title and contents } & \multicolumn{2}{|c|}{ FY 1992} & \multirow[b]{2}{*}{ Total } & \multirow{2}{*}{$\begin{array}{l}\text { Total } \\
\text { FY } 1985 \\
\text { to date }\end{array}$} & \multirow{2}{*}{$\begin{array}{l}\text { Remaining } \\
\text { copies }\end{array}$} \\
\hline & & Qtrs 1-2 & Qtrs 3-4 & & & \\
\hline $\begin{array}{l}\text { CONF- } \\
9006134\end{array}$ & $\begin{array}{l}\text { Global Climate Feedbacks: } \\
\text { Proceedings of the Brookhaven } \\
\text { National Laboratory Workshop } \\
\text { June 3-6, } 1990\end{array}$ & 35 & 7 & 42 & 112 & N/A \\
\hline TR001 & $\begin{array}{l}\text { On Possible Changes in } \\
\text { Global Sea Level and Their } \\
\text { Potential Causes, March } 1983\end{array}$ & 10 & 2 & 12 & 150 & 31 \\
\hline TR002 & $\begin{array}{l}\text { Effects of Approximate } \\
\text { Radiation Treatments Used in } \\
\text { the Climate Models on the } \\
\text { Clear Sky Thermal Radiation } \\
\text { Flux: Its Perturbation Due } \\
\text { to } \mathrm{CO}_{2} \text { Increase, January } 1983\end{array}$ & 1 & 2 & 3 & 93 & 15 \\
\hline TR003 & $\begin{array}{l}\text { Carbon Dioxide Emissions } \\
\text { from Fossil Fuels: A } \\
\text { Procedure for Estimation } \\
\text { and Results for 1950-1981, } \\
\text { June } 1983\end{array}$ & 14 & 6 & 20 & 195 & 46 \\
\hline TR004 & $\begin{array}{l}\text { Carbon in Live Vegetation } \\
\text { of Major World Ecosystems, } \\
\text { June } 1983\end{array}$ & 23 & 11 & 34 & 208 & 49 \\
\hline TR005 & $\begin{array}{l}\text { Deforestation Measured } \\
\text { by LANDSAT: Steps Toward } \\
\text { a Method June } 1983\end{array}$ & 11 & 4 & 15 & 206 & 21 \\
\hline TR006 & $\begin{array}{l}\text { Response of the North } \\
\text { American Corn Belt to } \\
\text { Climate Warming, August } 1983\end{array}$ & 10 & 7 & 17 & 141 & 41 \\
\hline TR007 & $\begin{array}{l}\text { An Analysis of Concepts } \\
\text { for Controlling Atmospheric } \\
\text { Carbon Dioxide }\end{array}$ & 16 & 9 & 25 & 293 & 12 \\
\hline TR008 & $\begin{array}{l}\text { Carbonate Chemistry of the } \\
\text { Weddell Sea }\end{array}$ & 3 & 2 & 5 & 111 & 20 \\
\hline TR009 & $\begin{array}{l}\text { Response of Unmanaged } \\
\text { Forests to } \mathrm{CO}_{2} \text {-Induced } \\
\text { Climate Change: Available } \\
\text { Information, Initial Tests, } \\
\text { and Data Requirements }\end{array}$ & 15 & 5 & 20 & 163 & 11 \\
\hline TR010 & $\begin{array}{l}\text { Computer Implementation of } \\
\text { a Globally Averaged Model } \\
\text { of the World Carbon Cycle }\end{array}$ & 14 & 4 & 18 & 200 & 173 \\
\hline
\end{tabular}


Table 3. (continued)

\begin{tabular}{|c|c|c|c|c|c|c|}
\hline \multirow{2}{*}{$\begin{array}{l}\text { Report } \\
\text { number }\end{array}$} & \multirow[b]{2}{*}{ Title and contents } & \multicolumn{2}{|c|}{ FY 1992} & \multirow{2}{*}{ Total } & \multirow{2}{*}{$\begin{array}{l}\text { Total } \\
\text { FY } 1985 \\
\text { to date }\end{array}$} & \multirow{2}{*}{$\begin{array}{c}\text { Remaining } \\
\text { copies }\end{array}$} \\
\hline & & Qtrs 1-2 & Qtrs 3-4 & & & \\
\hline TR011 & $\begin{array}{l}\text { Historical Carbon Dioxide: } \\
\text { Abundances Derived from the } \\
\text { Smithsonian Spectrobolograms }\end{array}$ & 28 & 1 & 29 & 132 & 24 \\
\hline TR012 & $\begin{array}{l}\text { Seasonal Climate Scenario } \\
\text { for Europe and North America } \\
\text { in the High- } \mathrm{CO}_{2} \text {, Warmer World }\end{array}$ & 10 & 5 & 15 & 141 & 29 \\
\hline TR013 & $\begin{array}{l}\text { An Analysis of Possible } \\
\text { Future Atmospheric } \\
\text { Retention of Fossil Fuel } \mathrm{CO}_{2}\end{array}$ & 9 & 2 & 11 & 202 & 40 \\
\hline TRO14 & $\begin{array}{l}\text { The Changing Pattern of } \\
\text { Fossil Fuel } \mathrm{CO}_{2} \text { Emissions }\end{array}$ & 11 & 5 & 16 & 201 & 19 \\
\hline TR015 & $\begin{array}{l}\text { A Proposed Reference Set } \\
\text { of Scenarios for } \\
\text { Radiatively Active } \\
\text { Atmospheric Constituents }\end{array}$ & 5 & 2 & 7 & 145 & 26 \\
\hline TR016 & $\begin{array}{l}\text { A . Systems Study for the } \\
\text { Removal, Recovery and } \\
\text { Disposal of Carbon Dioxide } \\
\text { from Fossil Fuel Power } \\
\text { Plants in the US. }\end{array}$ & 14 & 10 & 24 & 239 & 37 \\
\hline TR017 & $\begin{array}{l}\text { A Climatic Data Bank for } \\
\text { Northern Hemisphere Land } \\
\text { Areas, 1851-1980 }\end{array}$ & 10 & 4 & 14 & 217 & 99 \\
\hline TR018 & $\begin{array}{l}\text { A Global Paleoclimatic } \\
\text { Data Base for } 6000 \text { Year B.P. }\end{array}$ & 7 & 5 & 12 & 159 & 62 \\
\hline TR019 & $\begin{array}{l}\text { Carbon Dynamics of } \\
\text { Northern Hardwood Forests: } \\
\text { Gas Exchange Characteristics }\end{array}$ & 6 & 1 & 7 & 110 & 37 \\
\hline TR020 & $\begin{array}{l}\text { Reconstruction of Past } \\
\text { Atmospheric } \mathrm{CO}_{2} \text { Contents } \\
\text { from the Chemistry of the } \\
\text { Contemporary Ocean: An } \\
\text { Evaluation }\end{array}$ & 4 & 2 & 6 & 123 & 12 \\
\hline TR021 & $\begin{array}{l}\text { A Two Dimensional } \mathrm{CO}_{2^{-}} \\
\text {Ocean Model Including the } \\
\text { Biological Processes }\end{array}$ & 9 & 4 & 13 & 118 & 45 \\
\hline
\end{tabular}


Table 3. (continued)

\begin{tabular}{|c|c|c|c|c|c|c|}
\hline \multirow{2}{*}{$\begin{array}{l}\text { Report } \\
\text { number }\end{array}$} & \multirow[b]{2}{*}{ Title and contents } & \multicolumn{2}{|c|}{ FY 1992} & \multirow[b]{2}{*}{ Total } & \multirow{2}{*}{$\begin{array}{l}\text { Total } \\
\text { FY } 1985 \\
\text { to date }\end{array}$} & \multirow{2}{*}{$\begin{array}{l}\text { Remaining } \\
\text { copies }\end{array}$} \\
\hline & & Qtrs $1-2$ & Qtrs 3.4 & & & \\
\hline TR022 & $\begin{array}{l}\text { A Grid Point Surface Air } \\
\text { Temperature Data Set for } \\
\text { the Northern Hemisphere }\end{array}$ & 9 & 2 & 11 & 133 & 24 \\
\hline TR023 & $\begin{array}{l}\text { The Effect of Elevated } \\
\text { Atmospheric } \mathrm{CO}_{2} \text { on Plant } \\
\text { Communities }\end{array}$ & 10 & 10 & 20 & 198 & 22 \\
\hline TR024 & $\begin{array}{l}\text { Methods of Uncertainty } \\
\text { Analysis for a Global } \\
\text { Carbon Dioxide Model }\end{array}$ & 13 & 3 & 16 & 135 & 29 \\
\hline TR025 & $\begin{array}{l}\text { The Stability of Low- } \\
\text { Altitude Sea Surface } \\
\text { Temperatures: An Evaluation } \\
\text { of the CLIMAP Reconstruction } \\
\text { with Emphasis on the } \\
\text { Positive SST Anomalies }\end{array}$ & 4 & 2 & 6 & 77 & 55 \\
\hline TR026 & $\begin{array}{l}\text { Carbonate Chemistry of } \\
\text { the Bering Sea }\end{array}$ & 3 & 2 & 5 & 61 & 42 \\
\hline TR027 & $\begin{array}{l}\text { A Grid Point Surface Air } \\
\text { Temperature Data Set for } \\
\text { the Southern Hemisphere }\end{array}$ & 8 & 3 & 11 & 139 & 44 \\
\hline TR028 & $\begin{array}{l}\text { Definition and Characteri- } \\
\text { zation of Data Needs to } \\
\text { Describe the Potential } \\
\text { Effects of Increased Atmo- } \\
\text { spheric } \mathrm{CO}_{2} \text { on Marine } \\
\text { Fisheries from the } \\
\text { Northeast Pacific Ocean }\end{array}$ & 2 & 1 & 3 & 66 & 107 \\
\hline TR029 & $\begin{array}{l}\text { Preliminary Data Report for } \\
\text { the INDIGO1/INDIVAT } 3 \\
\text { Cruises in the Indian Ocean }\end{array}$ & 2 & 1 & 3 & 48 & 96 \\
\hline TR030 & $\begin{array}{l}\text { Effects of Energy Tech- } \\
\text { nology on Global } \mathrm{CO}_{2} \\
\text { Emissions }\end{array}$ & 14 & 9 & 23 & 354 & 33 \\
\hline TR031 & $\begin{array}{l}\text { Impact of Climate Change } \\
\text { from Increased Atmospheric } \\
\text { Carbon Dioxide on American } \\
\text { Agriculture }\end{array}$ & 12 & 11 & 23 & 332 & 9 \\
\hline TR032 & $\begin{array}{l}\text { A Comparison of Tropical } \\
\text { Forest Surveys }\end{array}$ & 11 & 5 & 16 & 149 & 61 \\
\hline
\end{tabular}


Table 3. (continued)

\begin{tabular}{|c|c|c|c|c|c|c|}
\hline \multirow{2}{*}{$\begin{array}{l}\text { Report } \\
\text { number }\end{array}$} & \multirow[b]{2}{*}{ Title and contents } & \multicolumn{2}{|c|}{ FY 1992} & \multirow[b]{2}{*}{ Total } & \multirow{2}{*}{$\begin{array}{l}\text { Total } \\
\text { FY } 1985 \\
\text { to date }\end{array}$} & \multirow{2}{*}{$\begin{array}{l}\text { Remaining } \\
\text { copies }\end{array}$} \\
\hline & & Qtrs 1-2 & Qtrs 3.4 & & & \\
\hline TR033 & $\begin{array}{l}\text { High Accuracy Standards } \\
\text { and Reference Methodology } \\
\text { for Carbon Dioxide in Air }\end{array}$ & 5 & 3 & 8 & 75 & 102 \\
\hline TR034 & $\begin{array}{l}\text { Carbonate Chemistry of the } \\
\text { North Pacific Ocean }\end{array}$ & 3 & 3 & 6 & 79 & 68 \\
\hline TR035 & $\begin{array}{l}\text { An Annotated Inventory of } \\
\text { Climatic Indices and Data } \\
\text { Sets }\end{array}$ & 12 & 6 & 18 & 209 & 22 \\
\hline \multirow[t]{2}{*}{ TR036 } & $\begin{array}{l}\text { Uncertainty in Future Global } \\
\text { Energy Use and Fossil Fuel } \\
\mathrm{CO}_{2} \text { Emissions } 1975 \text { to } 2075\end{array}$ & 11 & 10 & 21 & 310 & 210 \\
\hline & Appendices for TR036 & 4 & 13 & 17 & 58 & 19 \\
\hline TR037 & $\begin{array}{l}\text { Monthly Mean Pressure } \\
\text { Reconstructions for Europe } \\
\text { (Back to 1780) and North } \\
\text { America (to 1958) }\end{array}$ & 8 & 1 & 9 & 101 & 55 \\
\hline TR038 & $\begin{array}{l}\text { Data Bank of Antarctic } \\
\text { Surface Temperature and } \\
\text { Pressure Data }\end{array}$ & 9 & 1 & 10 & 59 & 116 \\
\hline TR039 & $\begin{array}{l}\text { The Prospect of Solving } \\
\text { the } \mathrm{CO}_{2} \text { Problem Through } \\
\text { Global Reforestation }\end{array}$ & 28 & 15 & 43 & 505 & 45 \\
\hline TR040 & $\begin{array}{l}\text { A Primer on Greenhouse } \\
\text { Gases }\end{array}$ & 31 & 16 & 47 & 1082 & 31 \\
\hline TR041 & $\begin{array}{l}\text { Regional Intercomparisons } \\
\text { of General Circulation } \\
\text { Model Predictions and } \\
\text { Historical Climate Data }\end{array}$ & 17 & 9 & 26 & 439 & 245 \\
\hline TR042 & $\begin{array}{l}\text { Surface Energy Balance of } \\
\text { Three General Circulation } \\
\text { Modeis: Current Climate and } \\
\text { Response to Increasing } \\
\mathrm{CO}_{2}\end{array}$ & 12 & 6 & 18 & 183 & 16 \\
\hline TR043 & $\begin{array}{l}\text { The Use of Statistical } \\
\text { Climate Crop Models for } \\
\text { Simulating Yield to Project } \\
\text { the Impacts of } \mathrm{CO}_{2} \text { Induced } \\
\text { Climate Change }\end{array}$ & 10 & 12 & 22 & 143 & 238 \\
\hline
\end{tabular}


Table 3. (continued)

\begin{tabular}{|c|c|c|c|c|c|c|}
\hline \multirow{2}{*}{$\begin{array}{l}\text { Report } \\
\text { number }\end{array}$} & \multirow[b]{2}{*}{ Title and contents } & \multicolumn{2}{|c|}{ FY 1992} & \multirow[b]{2}{*}{ Total } & \multirow{2}{*}{$\begin{array}{l}\text { Total } \\
\text { FY } 1985 \\
\text { to date }\end{array}$} & \multirow{2}{*}{$\begin{array}{l}\text { Remaining } \\
\text { copies }\end{array}$} \\
\hline & & Qtrs 1-2 & Qtrs 3-4 & & & \\
\hline TR044 & $\begin{array}{l}\text { Documentation of LAP Two- } \\
\text { Level Atmospheric General } \\
\text { Circulation Model }\end{array}$ & 5 & 5 & 10 & 58 & 20 \\
\hline TR045 & $\begin{array}{l}\text { A Preliminary Analysis of } \\
\text { U.S. } \mathrm{CO}_{2} \text { Emissions } \\
\text { Reduction Potential from } \\
\text { Energy Conservation and the } \\
\text { Substitution of Natural Gas } \\
\text { for Coal in the Period } \\
\text { to } 2010\end{array}$ & 15 & 15 & 30 & 577 & 482 \\
\hline TR046 & $\begin{array}{l}\text { Global Lake-Level Variations } \\
\text { from } 18,000 \text { to } 0 \text { Years Ago: } \\
\text { A Paleoclimatic Analysis }\end{array}$ & 13 & 6 & 19 & 111 & 194 \\
\hline TR047 & $\begin{array}{l}\text { An Evaluation of the Relation- } \\
\text { ship Between the Production } \\
\text { and Use of Energy and Atmo- } \\
\text { spheric Methane Emissions }\end{array}$ & 15 & 14 & 29 & 162 & 43 \\
\hline TR048 & $\begin{array}{l}\text { Effects of Air Temperature } \\
\text { on Atmospheric } \mathrm{CO}_{2} \text { Plant } \\
\text { Growth Relationships }\end{array}$ & 21 & 18 & 39 & 154 & N/A \\
\hline TR049 & $\begin{array}{l}\text { Simulating Climate with Two } \\
\text { Different Numerical Schemes }\end{array}$ & 10 & 7 & 17 & 59 & 121 \\
\hline TR050 & $\begin{array}{l}\text { Modeling } \mathrm{pCO}_{2} \text { in the Upper } \\
\text { Ocean: A Review of Relevant } \\
\text { Physical, Chemical, and } \\
\text { Biological Processes }\end{array}$ & 58 & 9 & 67 & 115 & N/A \\
\hline TR051 & $\begin{array}{l}\text { A Comprehensive } \\
\text { Precipitation Data Set } \\
\text { for Global Land Areas }\end{array}$ & 67 & 31 & 98 & 131 & 61 \\
\hline TR052 & $\begin{array}{l}\text { Processes for Identifying } \\
\text { Regional Influences of and } \\
\text { Responses to Increasing } \\
\text { Atmospheric } \mathrm{CO}_{2} \text { and Climate } \\
\text { Change-The } \mathrm{MINK} \text { Project. } \\
\text { This document is in eight } \\
\text { volumes (TR052A-TR052H) }\end{array}$ & 18 & 11 & 29 & 29 & $\begin{array}{r}89 \\
\text { Sets }\end{array}$ \\
\hline TR053 & $\begin{array}{l}\text { The Determination of Total } \\
\text { Dissolved Inorganic Carbon in } \\
\text { Sea Water Using Extraction/ } \\
\text { Coulometry: The First Stage } \\
\text { of a Collaborative Study }\end{array}$ & & 1 & 1 & 1 & 99 \\
\hline
\end{tabular}


Table 3. (continued)

\begin{tabular}{|c|c|c|c|c|c|c|}
\hline \multirow{2}{*}{$\begin{array}{l}\text { Report } \\
\text { number }\end{array}$} & \multirow[b]{2}{*}{ Title and contents } & \multicolumn{2}{|c|}{ FY 1992} & \multirow[b]{2}{*}{ Total } & \multirow{2}{*}{$\begin{array}{l}\text { Total } \\
\text { FY 1985 } \\
\text { to date }\end{array}$} & \multirow{2}{*}{$\begin{array}{l}\text { Remaining } \\
\text { copies }\end{array}$} \\
\hline & & Qtrs 1-2 & Qtrs 3-4 & & & \\
\hline TR054 & $\begin{array}{l}\text { Modeling the Response of Plants } \\
\text { and Ecosystems to Elevated } \mathrm{CO}_{2} \\
\text { and Climate Change }\end{array}$ & 1 & 48 & 49 & 49 & 63 \\
\hline $\begin{array}{c}\text { DOE/ER- } \\
0178\end{array}$ & $\begin{array}{l}\text { Carbon Dirxide Research } \\
\text { Plan - A Summary }\end{array}$ & 5 & 1 & 6 & 328 & 15 \\
\hline $\begin{array}{c}\text { DOE/ER- } \\
0186\end{array}$ & $\mathrm{CO}_{2}$ Climate Research Plan & 3 & 1 & 4 & 157 & 16 \\
\hline $\begin{array}{c}\text { DOE/ER- } \\
0187\end{array}$ & $\begin{array}{l}\text { Vegetable Response } \\
\text { Carbon Dioxide }\end{array}$ & 5 & 2 & 7 & 174 & 11 \\
\hline $\begin{array}{c}\text { DOE/ER- } \\
0188\end{array}$ & Carbon Cycle Research Plan & 5 & 1 & 6 & 108 & 12 \\
\hline $\begin{array}{c}\text { DOE/ER- } \\
0235\end{array}$ & $\begin{array}{l}\text { Detecting the Climatic } \\
\text { Effects of Increasing } \\
\text { Carbon Dioxide } \\
\text { (Hardbound }=\mathrm{HB} \text { ) }\end{array}$ & 39 & 1 & 40 & 521 & 7 \\
\hline $\begin{array}{c}\text { DOE/ER- } \\
0235\end{array}$ & $\begin{array}{l}\text { Detecting the Climatic } \\
\text { Effects of Increasing } \\
\text { Carbon Dioxide } \\
\text { (Softbound = SB) }\end{array}$ & 15 & 16 & 31 & 1598 & 742 \\
\hline $\begin{array}{c}\text { DOE/ER- } \\
0236\end{array}$ & $\begin{array}{l}\text { Characterization of } \\
\text { Information Requirements } \\
\text { for Studies of } \mathrm{CO}_{2} \text { Effects: } \\
\text { Water Resources, Agriculture, } \\
\text { Fisheries, Forests and Human } \\
\text { Health (HB) }\end{array}$ & 41 & 13 & 54 & 537 & 78 \\
\hline $\begin{array}{c}\text { DOE/ER- } \\
0236\end{array}$ & $\begin{array}{l}\text { Characterization of } \\
\text { Information Requirements } \\
\text { for Studies of } \mathrm{CO}_{2} \text { Effects: } \\
\text { Water Resources, Agriculture, } \\
\text { Fisheries, Forests and Human } \\
\text { Health (SB) }\end{array}$ & 3 & 4 & 7 & 1680 & N/A \\
\hline $\begin{array}{c}\text { DOE/ER- } \\
0237\end{array}$ & $\begin{array}{l}\text { Projecting the Climatic } \\
\text { Effects of Increasing } \\
\text { Carbon Dioxide (HB) }\end{array}$ & 39 & 1 & 40 & 521 & 10 \\
\hline $\begin{array}{c}\text { DOE/ER - } \\
0237\end{array}$ & $\begin{array}{l}\text { Projecting the Climatic } \\
\text { Effects of Increasing } \\
\text { Carbon Dioxide (SB) }\end{array}$ & 14 & 15 & 29 & 1627 & 143 \\
\hline
\end{tabular}


Table 3. (continued)

\begin{tabular}{|c|c|c|c|c|c|c|}
\hline \multirow{2}{*}{$\begin{array}{l}\text { Report } \\
\text { number }\end{array}$} & \multirow[b]{2}{*}{ Title and contents } & \multicolumn{2}{|c|}{ FY 1992} & \multirow[b]{2}{*}{ Total } & \multirow{2}{*}{$\begin{array}{l}\text { Total } \\
\text { FY } 1985 \\
\text { to date }\end{array}$} & \multirow{2}{*}{$\begin{array}{l}\text { Remaining } \\
\text { copies }\end{array}$} \\
\hline & & Qtrs $1-2$ & Qtrs 3-4 & & & \\
\hline $\begin{array}{c}\text { DOE/ER- } \\
0238\end{array}$ & $\begin{array}{l}\text { Direct Effects of } \\
\text { Increasing Carbon Dioxide } \\
\text { on Vegetation (HB) }\end{array}$ & 40 & 1 & 41 & 522 & 15 \\
\hline $\begin{array}{c}\text { DOE/ER- } \\
0238\end{array}$ & $\begin{array}{l}\text { Direct Effects of } \\
\text { Increasing Carbon Dioxide } \\
\text { on Vegetation (SB) }\end{array}$ & 35 & 17 & 52 & 1682 & 129 \\
\hline $\begin{array}{c}\text { DOE/ER- } \\
0239\end{array}$ & $\begin{array}{l}\text { Atmospheric Carbon Dioxide } \\
\text { and the Global Carbon Cycle } \\
\text { (HB) }\end{array}$ & 36 & 1 & 37 & 516 & 16 \\
\hline $\begin{array}{c}\text { DOE/ER- } \\
0239\end{array}$ & $\begin{array}{l}\text { Atmospheric Carbon Dioxide } \\
\text { and the Global Carbon Cycle } \\
\text { (SB) }\end{array}$ & 27 & 18 & 45 & 1693 & 46 \\
\hline $\begin{array}{c}\text { DOE/ER- } \\
0316\end{array}$ & $\begin{array}{l}\text { Master Index of the Carbon } \\
\text { Dioxide Research State-of- } \\
\text { the-Art Report Series (HB) }\end{array}$ & 37 & 2 & 39 & 566 & 85 \\
\hline $\begin{array}{l}\text { DOE/ER- } \\
0316\end{array}$ & $\begin{array}{l}\text { Master Index of the Carbon } \\
\text { Dioxide Research State-of- } \\
\text { the-Art Report Series (SB) }\end{array}$ & 14 & 10 & 24 & 764 & 272 \\
\hline $\begin{array}{c}\mathrm{DOE} / \mathrm{EV} \text { - } \\
0202\end{array}$ & $\begin{array}{l}\text { Carbon Dioxide and Climate: } \\
\text { Summaries of Research in } \\
\text { FY } 1983 \text { and FY } 1984\end{array}$ & 5 & 1 & 6 & 153 & 15 \\
\hline $\begin{array}{c}\text { DOE/EV. } \\
0202 / 1\end{array}$ & $\begin{array}{l}\text { Carbon Dioxide and Climate: } \\
\text { Summaries of Research in } \\
\text { FY } 1985\end{array}$ & 3 & 1 & 4 & 113 & 17 \\
\hline $\begin{array}{c}\text { DOE/ER- } \\
0299\end{array}$ & $\begin{array}{l}\text { Carbon Dioxide Summaries } \\
\text { of Research in FY } 1986\end{array}$ & 3 & 1 & 4 & 59 & 18 \\
\hline $\begin{array}{r}\text { DOE/EV- } \\
0129\end{array}$ & $\begin{array}{l}\text { Research Issues and } \\
\text { Supporting Research of the } \\
\text { National Program on Carbon } \\
\text { Dioxide, Environment and } \\
\text { Society - FY } 1980\end{array}$ & & & & 9 & 19 \\
\hline $\begin{array}{c}\mathrm{DOE} / \mathrm{ER}- \\
0347\end{array}$ & $\begin{array}{l}\text { Carbon Dioxide \& Climate: } \\
\text { Summaries of Research in } \\
\text { FY } 1987\end{array}$ & 3 & 1 & 4 & 190 & 12 \\
\hline $\begin{array}{c}\text { DOE/ER- } \\
0385\end{array}$ & $\begin{array}{l}\text { Carbon Dioxide \& Climate: } \\
\text { Summaries of Research in } \\
\text { FY } 1988\end{array}$ & & 1 & 1 & 144 & N/A \\
\hline
\end{tabular}


Table 3. (continued)

\begin{tabular}{|c|c|c|c|c|c|c|}
\hline \multirow{2}{*}{$\begin{array}{l}\text { Report } \\
\text { number }\end{array}$} & \multirow[b]{2}{*}{ Title and contents } & \multicolumn{2}{|c|}{ FY 1992} & \multirow[b]{2}{*}{ Total } & \multirow{2}{*}{$\begin{array}{l}\text { Total } \\
\text { FY } 1985 \\
\text { to date }\end{array}$} & \multirow{2}{*}{$\begin{array}{l}\text { Remaining } \\
\text { copies }\end{array}$} \\
\hline & & Qtrs 1-2 & Qtrs 3-4 & & & \\
\hline $\begin{array}{c}\text { DOE/ER- } \\
0406\end{array}$ & $\begin{array}{l}\text { Global Distribution of } \\
\text { Total Cloud Cover and } \\
\text { Cloud Type Amounts Over } \\
\text { the Ocean }\end{array}$ & 10 & 6 & 16 & 82 & 168 \\
\hline $\begin{array}{c}\text { DOE/ER- } \\
0411\end{array}$ & $\begin{array}{l}\text { Atmospheric Carbon Dioxide } \\
\text { and the Greenhouse Effect }\end{array}$ & 18 & 30 & 48 & 7248 & N/A \\
\hline $\begin{array}{c}\text { DOE/ER- } \\
0425\end{array}$ & $\begin{array}{l}\text { Carbon Dioxide and Climate: } \\
\text { Summaries of Research in } \\
\text { FY } 1989\end{array}$ & 7 & 2 & 9 & 219 & 13 \\
\hline $\begin{array}{c}\text { DOE/ER- } \\
0441\end{array}$ & $\begin{array}{l}\text { Atmospheric Radiation } \\
\text { Measurement Program Plan }\end{array}$ & 159 & 43 & 202 & 938 & 225 \\
\hline $\begin{array}{c}\text { DOE/ER } \\
0442\end{array}$ & $\begin{array}{l}\text { Atmospheric Radiation } \\
\text { Measurement Program Plan } \\
\text { (Executive Summary) }\end{array}$ & 125 & 77 & 202 & 996 & 678 \\
\hline $\begin{array}{r}\text { DOE/ER- } \\
0470 \mathrm{~T}\end{array}$ & $\begin{array}{l}\text { Carbon Dioxide and Climate: } \\
\text { Summaries of Research in } \\
\text { FY } 1990\end{array}$ & 18 & 1 & 19 & 229 & 453 \\
\hline $\begin{array}{r}\text { DOE/ER }- \\
0479 \mathrm{~T}\end{array}$ & $\begin{array}{l}\text { Building an Advanced } \\
\text { Climate Model Program } \\
\text { Plan for the CHAMMP } \\
\text { Climate Modeling Program }\end{array}$ & 66 & 8 & 74 & 218 & 127 \\
\hline $\begin{array}{l}\text { DOE/ER - } \\
0494 \mathrm{~T}\end{array}$ & $\begin{array}{l}\text { Identification, } \\
\text { Recommendation, and } \\
\text { Justification of } \\
\text { Potential Locales for } \\
\text { ARM sites (Exec. Summ.) }\end{array}$ & 22 & 41 & 63 & 102 & 191 \\
\hline $\begin{array}{r}\text { DOE/ER- } \\
0495 \mathrm{~T}\end{array}$ & $\begin{array}{l}\text { Identification, } \\
\text { Recommendation, and } \\
\text { Justification of } \\
\text { Potential Locales for } \\
\text { ARM sites }\end{array}$ & 36 & 42 & 78 & 117 & 176 \\
\hline $\begin{array}{r}\text { DOE/ER- } \\
0508 \mathrm{~T}\end{array}$ & $\begin{array}{l}\text { Carbon Dioxide and } \\
\text { Clirnate: Summaries } \\
\text { of Research in FY } 1991\end{array}$ & 58 & $23 ;$ & 294 & 294 & 26 \\
\hline $\begin{array}{r}\text { DOE/ER- } \\
0549 \mathrm{~T}\end{array}$ & $\begin{array}{l}\text { Multi-Year Strategic Plan } \\
\text { for the Atmospheric Studies } \\
\text { in Complex Terrain (ASCOT) } \\
\text { Program }\end{array}$ & & 2 & 2 & 2 & 180 \\
\hline
\end{tabular}


Table 3. (continued)

\begin{tabular}{|c|c|c|c|c|c|c|}
\hline \multirow{2}{*}{$\begin{array}{l}\text { Report } \\
\text { number }\end{array}$} & \multirow[b]{2}{*}{ Title and contents } & \multicolumn{2}{|c|}{ FY 1992} & \multirow[b]{2}{*}{ Total } & \multirow{2}{*}{$\begin{array}{l}\text { Total } \\
\text { FY } 1985 \\
\text { to date }\end{array}$} & \multirow{2}{*}{$\begin{array}{l}\text { Remaining } \\
\text { copies }\end{array}$} \\
\hline & & Qtrs 1-2 & Qtrs 3-4 & & & \\
\hline $\begin{array}{r}\text { DOE/EV- } \\
60235-1\end{array}$ & $\begin{array}{l}\text { Glaciers, Ice Sheets, and } \\
\text { Sea Level: Effect of a } \mathrm{CO}_{2^{-}} \\
\text {Induced Climatic Change (HB) }\end{array}$ & 39 & 1 & 40 & 521 & 105 \\
\hline $\begin{array}{r}\text { DOE/EV- } \\
60235-1\end{array}$ & $\begin{array}{l}\text { Glaciers, Ice Sheets, and } \\
\text { Sea Level: Effect of a } \mathrm{CO}_{2^{-}} \\
\text {Induced Climatic Change (SB) }\end{array}$ & 11 & 10 & 21 & 1104 & 179 \\
\hline $\begin{array}{l}\text { DOE/ER- } \\
60085-\mathrm{H} 1\end{array}$ & $\begin{array}{l}\text { Global Distribution of } \\
\text { Total Cloud Cover and Cloud } \\
\text { Type Amounts Over Land }\end{array}$ & 11 & 7 & 18 & 161 & 140 \\
\hline $\begin{array}{l}\text { DOE/ER- } \\
60197-H 1\end{array}$ & $\begin{array}{l}\text { On the Surging Potential of } \\
\text { Polar Ice Streams FY } 1987 \\
\text { Amounts Over Land }\end{array}$ & 5 & 3 & 8 & 41 & 184 \\
\hline $\begin{array}{c}\text { DOE/ER- } \\
0094 \mathrm{P}\end{array}$ & $\begin{array}{l}\text { Global Climate Trends and } \\
\text { Greenhouse Gas Data: } \\
\text { Federal Activities in Data } \\
\text { Collection, Archiving, and } \\
\text { Dissemination }\end{array}$ & 13 & 4 & 17 & 93 & 57 \\
\hline PNL-4384 & $\begin{array}{l}\text { An Analysis of Methods and } \\
\text { Models for Assessing the Direct } \\
\text { and Indirect Economic Impacts } \\
\text { of } \mathrm{CO}_{2} \text {-Induced Environmental } \\
\text { Changes in the Agricultural } \\
\text { Sector of the U.S. Economy }\end{array}$ & & & & 38 & N/A \\
\hline PNL-4709 & $\begin{array}{l}\text { Identification and Preliminary } \\
\text { Characterization of Global } \\
\text { Water Resource Issues Which } \\
\text { May Be Affected by } \mathrm{CO}_{2-} \\
\text { Induced Climate Change }\end{array}$ & & & & 51 & N/A \\
\hline BERN & Bern $\mathrm{CO}_{2}$ Symposium & 3 & 2 & 5 & 89 & 14 \\
\hline $\begin{array}{r}\text { DOE/NBB- } \\
0039\end{array}$ & $\begin{array}{l}\text { International Carbon } \\
\text { Dioxide-Related Activities: } \\
\text { The International } \\
\text { Organizations Involved and } \\
\text { U.S. Bilateral Arrangements }\end{array}$ & 1 & 1 & 2 & 37 & N/A \\
\hline $\begin{array}{c}\text { DOE/NBB- } \\
0068\end{array}$ & $\begin{array}{l}\text { Review of Carbon Dioxide } \\
\text { Research Staffing and } \\
\text { Academic Support }\end{array}$ & 1 & 2 & 3 & 42 & 65 \\
\hline DA001 & $\begin{array}{l}\text { Field Studies of Plant Responses } \\
\text { to Elevated Carbon Dioxide } \\
\text { Levels, } 1980\end{array}$ & 4 & 5 & 9 & 48 & 5 \\
\hline
\end{tabular}


Table 3. (continued)

\begin{tabular}{|c|c|c|c|c|c|c|}
\hline \multirow{2}{*}{$\begin{array}{l}\text { Report } \\
\text { number }\end{array}$} & \multirow[b]{2}{*}{ Title and contents } & \multicolumn{2}{|c|}{ FY 1992} & \multirow[b]{2}{*}{ Total } & \multirow{2}{*}{$\begin{array}{l}\text { Total } \\
\text { FY } 1985 \\
\text { to date }\end{array}$} & \multirow{2}{*}{$\begin{array}{l}\text { Remaining } \\
\text { copies }\end{array}$} \\
\hline & & Qtrs 1-2 & Qtrs 3-4 & & & \\
\hline $\mathrm{DA} 002$ & $\begin{array}{l}\text { The Soybean Crop Simulator, } \\
\text { Glycim: Model Documentation, } \\
1982\end{array}$ & & & & 13 & 13 \\
\hline DA003 & $\begin{array}{l}\text { Effects of Increased Carbon } \\
\text {.Dioxide on Photosynthesis } \\
\text { and Agricultural Produc- } \\
\text { tivity of Soybeans, } 1981\end{array}$ & 6 & 4 & 10 & 44 & 4 \\
\hline DA004 & $\begin{array}{l}\text { Soybean Responses to Carbon } \\
\text { Dioxide: Measurement and } \\
\text { Simulation, } 1981\end{array}$ & & & & 14 & 12 \\
\hline DA005 & $\begin{array}{l}\text { Field Studies of Plant } \\
\text { Responses to Elevated Carbon } \\
\text { Dioxide Levels, } 1981\end{array}$ & & & & 12 & 13 \\
\hline DA006 & $\begin{array}{l}\text { Effects of Carbon Dioxide } \\
\text { Enrichment on Nitrogen Fix- } \\
\text { ation in Soybeans, } 1981\end{array}$ & & & & 9 & 7 \\
\hline DA007 & $\begin{array}{l}\text { Effects of Increased Carbon } \\
\text { Dioxide on Photosynthesis, } \\
\text { Transpiration, Water-Use } \\
\text { Efficiency, and Productivity } \\
\text { of Soybeans, } 1982\end{array}$ & & & & 10 & N/A \\
\hline DA008 & $\begin{array}{l}\text { Soybean Responses to Carbon } \\
\text { Dioxide: Measurement and } \\
\text { Simulation, } 1982\end{array}$ & & & & 14 & N/A \\
\hline DA009 & $\begin{array}{l}\text { Field Studies of Plant Re- } \\
\text { sponses to Elevated Carbon } \\
\text { Dioxide Levels, } 1982\end{array}$ & & & & 7 & N/A \\
\hline DA010 & $\begin{array}{l}\text { Effects of Carbon Dioxide } \\
\text { Enrichment on Nitrogen Fixa- } \\
\text { tion in Soybeans, } 1982\end{array}$ & 2 & & 2 & 9 & N/A \\
\hline DA011 & $\begin{array}{l}\text { The Simulation, with GLYCIM, } \\
\text { of Soybean Crops Grown in } \\
\text { the Field and at Various } \mathrm{CO}_{2} \\
\text { Concentrations in Open-Top } \\
\text { Chambers During } 1982\end{array}$ & & & & 8 & 6 \\
\hline DA012 & $\begin{array}{l}\text { Field Studies of Plant } \\
\text { Responses to Elevated Carbon } \\
\text { Dioxide Levels, } 1983\end{array}$ & & & & 12 & N/A \\
\hline
\end{tabular}


Table 3. (continued)

\begin{tabular}{|c|c|c|c|c|c|c|}
\hline \multirow{2}{*}{$\begin{array}{l}\text { Report } \\
\text { number }\end{array}$} & \multirow[b]{2}{*}{ Title and contents } & \multicolumn{2}{|c|}{ FY 1992} & \multirow{2}{*}{ Total } & \multirow{2}{*}{$\begin{array}{l}\text { Total } \\
\text { FY } 1985 \\
\text { to date }\end{array}$} & \multirow{2}{*}{$\begin{array}{l}\text { Remaining } \\
\text { copies }\end{array}$} \\
\hline & & Qtrs 1.2 & Qtrs 3-4 & & & \\
\hline DA014 & $\begin{array}{l}\text { Effects of Increased Carbon } \\
\text { Dioxide and Water Stress } \\
\text { Interactions on Photo- } \\
\text { synthesis, Transpiration, } \\
\text { and Productivity of } \\
\text { Soybeans, } 1983\end{array}$ & & & & 4 & N/A \\
\hline DA019 & $\begin{array}{l}\text { The Response of Arctic Eco- } \\
\text { systems to Elevated Carbon } \\
\text { Dioxide Regimes, } 1984\end{array}$ & & & & 15 & 6 \\
\hline DA020 & $\begin{array}{l}\text { Radiation Densitometry in } \\
\text { Tree-Ring Analysis: A Review } \\
\text { and Procedure Manual, } 1985\end{array}$ & & & & 44 & 10 \\
\hline DA021 & $\begin{array}{l}\text { Effects of Increasing } \\
\text { Atmospheric } \mathrm{CO}_{2} \text { on the } \\
\text { Yield and Water Use of } \\
\text { Crops, } 1983\end{array}$ & & & & 9 & N/A \\
\hline DA022 & $\begin{array}{l}\text { Field Studies of Sweet } \\
\text { Potatoes and Cowpeas in } \\
\text { Response to Elevated } \\
\text { Carbon Dioxide, } 1985\end{array}$ & 5 & 2 & 7 & 49 & 15 \\
\hline DA023 & $\begin{array}{l}\text { Effects of Increasing } \\
\text { Atmospheric } \mathrm{CO}_{2} \text { on the Yield } \\
\text { and Water Use of Crops, } 1984\end{array}$ & & & & 13 & 16 \\
\hline DA024 & $\begin{array}{l}\text { Sorghum at Elevated Levels } \\
\text { of } \mathrm{CO}_{2}\end{array}$ & & & & 12 & 7 \\
\hline DA025 & $\begin{array}{l}\text { Preliminary Ring-Width and } \\
\text { Ring-Density Data for } \\
\text { Deriving Wood Mass } \\
\text { Chronologies of Coniferous } \\
\text { Species from the Northwest } \\
\text { U.S. and Canada }\end{array}$ & & & & 5 & 9 \\
\hline DA026 & $\begin{array}{l}\text { Western U.S. Tree-Ring } \\
\text { Index Chronology Data for } \\
\text { Detection of Arboreal } \\
\text { Response to Increasing } \\
\text { Carbon Dioxide }\end{array}$ & & & & 10 & N/A \\
\hline DA027 & $\begin{array}{l}\text { Western U.S. Tree-Ring } \\
\text { Index Chronology Data for } \\
\text { Detection of Arboreal } \\
\text { Response to Increasing } \\
\text { Carbon Dioxide }\end{array}$ & & & & 6 & N/A \\
\hline
\end{tabular}


Table 3. (continued)

\begin{tabular}{|c|c|c|c|c|c|c|}
\hline \multirow{2}{*}{$\begin{array}{l}\text { Report } \\
\text { number }\end{array}$} & \multirow[b]{2}{*}{ Title and contents } & \multicolumn{2}{|c|}{ FY 1992} & \multirow[b]{2}{*}{ Total } & \multirow{2}{*}{$\begin{array}{l}\text { Total } \\
\text { FY } 1985 \\
\text { to date }\end{array}$} & \multirow{2}{*}{$\begin{array}{c}\text { Remaining } \\
\text { copies }\end{array}$} \\
\hline & & Qtrs 1.2 & Qtrs 3-4 & & & \\
\hline DA028 & $\begin{array}{l}\text { Assessing the Effects of } \\
\text { Elevated Carbon Dioxide } \\
\text { on Plants: Towards the } \\
\text { Development of a Generic } \\
\text { Plant Growth Model }\end{array}$ & & 1 & 1 & 18 & 31 \\
\hline DA033 & $\begin{array}{l}\text { Influence of Nutrition } \\
\text { on Vegetation Response } \\
\text { to Carbon Dioxide. I. } \\
\text { Interactions of Nitrogen } \\
\text { and Phosphorus Supply } \\
\text { on Soybean Growth and } \\
\text { Nutritional Parameters, } \\
1986\end{array}$ & & & & 6 & N/A \\
\hline DA034 & $\begin{array}{l}\text { Effects of Elevated Carbon } \\
\text { Dioxide on Chesapeake Bay } \\
\text { Wetlands. I. Description } \\
\text { of the Study Site }\end{array}$ & & & & 4 & N/A \\
\hline DA040 & $\begin{array}{l}\text { Effect of Elevated Levels } \\
\text { of } \mathrm{CO}_{2} \text { on Winter Wheat } \\
\text { Under Two Moisture } \\
\text { Regimes }\end{array}$ & & & & 1 & N/A \\
\hline DA041 & $\begin{array}{l}\text { SERECO: A Model for the } \\
\text { Simulation of Ecosystem } \\
\text { Response to Elevated } \mathrm{CO}_{2} \\
\text { Parts I - III }\end{array}$ & & & & 1 & N/A \\
\hline DA042 & $\begin{array}{l}\text { PRECO: A Model for the } \\
\text { Simulation of Plant } \\
\text { Response to Elevated } \mathrm{CO}_{2} \\
\text { Parts I - III }\end{array}$ & & & & 7 & N/A \\
\hline & Total & 1,769 & 1,067 & 2,836 & 38,813 & \\
\hline
\end{tabular}

N/A - No longer available from CDIAC 
Table 4. CDIAC reports distribution

\begin{tabular}{|c|c|c|c|c|c|c|}
\hline \multirow{2}{*}{$\begin{array}{l}\text { Report } \\
\text { number }\end{array}$} & \multirow[b]{2}{*}{ Title and contents } & \multicolumn{2}{|c|}{ FY 1992} & \multirow{2}{*}{ Total } & \multirow{2}{*}{$\begin{array}{l}\text { Total } \\
\text { FY } 1985 \\
\text { to date }\end{array}$} & \multirow{2}{*}{$\begin{array}{l}\text { Remaining } \\
\text { copies }\end{array}$} \\
\hline & & Qtrs 1-2 & Qtrs 3-4 & & & \\
\hline $\begin{array}{r}\text { CDIAC- } \\
13\end{array}$ & $\begin{array}{l}\text { Direct Effects of Atmos- } \\
\text { pheric } \mathrm{CO}_{2} \text { Enrichment on } \\
\text { Plants and Ecosystems: } \\
\text { A Bibliography with Abstracts, } \\
\text { June } 1986\end{array}$ & & 2 & 2 & 18 & 37 \\
\hline $\begin{array}{r}\text { CDIAC- } \\
21\end{array}$ & $\begin{array}{l}\text { Graduate Student Theses } \\
\text { Supported by Carbon Dioxide } \\
\text { Research Division Office of } \\
\text { Basic Energy Sciences U.S. } \\
\text { Department of Energy, } \\
\text { September } 1987\end{array}$ & 5 & 2 & 7 & 26 & 14 \\
\hline $\begin{array}{r}\text { CDIAC- } \\
23\end{array}$ & $\begin{array}{l}\text { Preliminary Development of } \\
\text { a Seashore-Effects Analysis } \\
\text { System, February } 1989\end{array}$ & 5 & 2 & 7 & 38 & 23 \\
\hline $\begin{array}{l}\text { CDIAC- } \\
24 / \mathrm{V} 1\end{array}$ & $\begin{array}{l}\text { Bibliography on Tropical } \\
\text { Rain Forests and the Global } \\
\text { Carbon Cycle. Vol. 1. An } \\
\text { Introduction to the Literature, } \\
\text { May } 1988\end{array}$ & 15 & 10 & 25 & 91 & 54 \\
\hline $\begin{array}{l}\text { CDIAC- } \\
24 / \mathrm{V} 2\end{array}$ & $\begin{array}{l}\text { Bibliography on Tropical } \\
\text { Rain Forests and the Global } \\
\text { Carbon Cycle. Vol. } 2 . \\
\text { South Asia, February } 1989\end{array}$ & 12 & 7 & 19 & 88 & 108 \\
\hline $\begin{array}{r}\text { CDIAC- } \\
28\end{array}$ & $\begin{array}{l}\text { Environmental Consequences } \\
\text { of } \mathrm{CO}_{2} \text {-Climate Interactions: } \\
\text { The Need for Integrated } \\
\text { Resource Analysis, January } 1989\end{array}$ & 10 & 3 & 13 & 78 & 16 \\
\hline $\begin{array}{r}\text { CDIAC- } \\
32\end{array}$ & $\begin{array}{l}\text { A Plan for Intermodel } \\
\text { Comparison of Atmospheric } \\
\mathrm{CO}_{2} \text { Projections with } \\
\text { Uncertainty Analysis, June } 1990\end{array}$ & 24 & 7 & 31 & 45 & 54 \\
\hline $\begin{array}{r}\text { CDIAC- } \\
33\end{array}$ & $\begin{array}{l}\text { Environmental Atlas of the } \\
\text { Iowa-Kansas-Missouri-Nebraska } \\
\text { Climate-Change Study Region, } \\
\text { March } 1990\end{array}$ & 1 & 2 & 3 & 12 & 8 \\
\hline $\begin{array}{r}\text { CDIAC- } \\
34\end{array}$ & $\begin{array}{l}\text { Catalog of Data Bases } \\
\text { and Reports }\end{array}$ & 427 & 137 & 564 & 4376 & a \\
\hline $\begin{array}{l}\text { CDLAC- } \\
34 / R 2\end{array}$ & $\begin{array}{l}\text { Catalog of Data Bases } \\
\text { and Reports }\end{array}$ & & 409 & 409 & 409 & $\mathrm{a}$ \\
\hline
\end{tabular}


Table 4. (continued)

\begin{tabular}{|c|c|c|c|c|c|c|}
\hline \multirow{2}{*}{$\begin{array}{l}\text { Report } \\
\text { number }\end{array}$} & \multirow[b]{2}{*}{ Title and contents } & \multicolumn{2}{|c|}{ FY 1992} & \multirow[b]{2}{*}{ Total } & \multirow{2}{*}{$\begin{array}{l}\text { Total } \\
\text { FY } 1985 \\
\text { to date }\end{array}$} & \multirow{2}{*}{$\begin{array}{l}\text { Remaining } \\
\text { copies }\end{array}$} \\
\hline & & Qtrs 1-2 & Qtrs 3-4 & & & \\
\hline $\begin{array}{r}\text { CDLAC- } \\
36\end{array}$ & $\begin{array}{l}\text { Trends '90: A Compendium } \\
\text { of Data on Global Change, } \\
\text { August } 1990\end{array}$ & 415 & 155 & 570 & 8202 & N/A \\
\hline $\begin{array}{r}\text { CDLAC- } \\
38\end{array}$ & $\begin{array}{l}\text { Documentation and Analysis } \\
\text { of a Global } \mathrm{CO}_{2} \text { Model. } \\
\text { Developed by Peng et Al. } \\
\text { (1983), June } 1990\end{array}$ & 9 & 4 & 13 & 50 & 67 \\
\hline $\begin{array}{r}\text { CDIAC- } \\
39\end{array}$ & $\begin{array}{l}\text { Glossary: Carbon Dioxide } \\
\text { and rlimate, August } 1990\end{array}$ & 760 & 506 & 1266 & 3363 & 2637 \\
\hline $\begin{array}{r}\text { CDIAC } \\
46\end{array}$ & $\begin{array}{l}\text { Trend's } 91 \text { : A Compendium } \\
\text { of Data on Global Change, } \\
\text { December } 1991\end{array}$ & 234 & 1457 & 1691 & 1691 & 1075 \\
\hline $\begin{array}{r}\text { CDLAC. } \\
48\end{array}$ & $\begin{array}{l}\text { Carbon Dioxide Information } \\
\text { Analysis Center: FY } 1991 \\
\text { Activities }\end{array}$ & & 103 & 103 & 103 & \\
\hline $\begin{array}{r}\text { CDLAC- } \\
49\end{array}$ & $\begin{array}{l}\text { Trends 91: A Compendium } \\
\text { of Data on Global Change, } \\
\text { Highlights }\end{array}$ & & 239 & 239 & 239 & \\
\hline \multirow[t]{6}{*}{$\begin{array}{r}\text { CDLAC- } \\
52\end{array}$} & $\begin{array}{l}\text { Climate Change and Water } \\
\text { Supply, Management and Use: } \\
\text { A Literature Review }\end{array}$ & & 2 & 2 & 2 & \\
\hline & CDLAC Communications & 624 & 916 & 1540 & 3883 & b \\
\hline & ARM Outreach newsletter & & 154 & 154 & 154 & \\
\hline & CDIAC Factsheets & 646 & 124 & 770 & 6126 & a \\
\hline & DOE Researsh Summaries & 890 & 1821 & 2711 & 2711 & \\
\hline & Total & 4,077 & 6,062 & 10,139 & 33,705 & \\
\hline
\end{tabular}

'These documents are updated periodically and reprinted to satisfy requests

${ }^{b} \mathrm{New}$ issues produced several times a year 


\section{World Directory of $\mathrm{CO}_{2}$ Researchers and Policymakers}

Developing and maintaining an active inventory of researchers, policy scientists, administrators, officials, educators, librarians and communications specialists is an essential activity for the successful dissemination of CDIAC's derived-information products, publications, and other services. Over the course of six year's efforts, CDIAC has compiled such an inventory in its World Directory of $\mathrm{CO}_{2}$ Researchers and Policymakers. This inventory consists of 7,515 individuals in 150 countries (Fig. 10). Table 5 details activities in CDIAC's World Directory. Universities (35\%) and government agencies (33\%) comprise the two largest categories in the World Directory (Fig. 11). Names were added to the directory from key national and international climate-change committees and working groups, lists of conference and seminar attendees (from the traditional scientific communities but also, as the topic is rapidly expanding, in the social sciences and humanities disciplines), and other directories of potential CDIAC users (e.g., state environmental agency staff, electric utility managers, special libraries, environmental media specialists and reporters, special and public inierest groups). This directory is used primarily as an in-house refersnce by program managers and sponsors. Special, limited arrangements have been made for sharing dire::iory information with persons and organizations outside CDIAC or DOE.

Total participants at close of FY $1992=7,515$

Table 5. Net additions in total participants

\begin{tabular}{lccr}
\hline & \multicolumn{2}{c}{ Quarter FY 1992 } & \\
\cline { 2 - 3 } Category & Qtrs 1-2 & Qtrs 3-4 & Total \\
\hline $\begin{array}{l}\text { U.S. Members } \\
\begin{array}{l}\text { Foreign embassies } \\
\text { (in United States) }\end{array}\end{array}$ & 179 & 364 & 743 \\
$\begin{array}{l}\text { Foreign members } \\
(143 \text { countries) }\end{array}$ & 134 & 251 & 1 \\
Net Additions & 514 & 615 & 1,129 \\
\hline Total Additions & 559 & 921 & 1,480 \\
$\quad$ Total Deletions & 45 & 306 & 351 \\
\hline
\end{tabular}

The format of the World Directory was changed to reduce the costs of publication and distribution. The Directory was produced as a single volume with United States and foreign entries identified in Appendices A and $\mathrm{B}$, respectively. All phone numbers, FAX numbers, and eMail addresses, when available, have been included with each name and address entry. The Foreign and Telephone directories previously produced are now combined in one Comprehensive Directory. 
Table 6. Alphabetical listing of countries and their outlying territories and the number of participants listed in the World Directory of Researchers and Policymakers

September 1992

\begin{tabular}{|c|c|c|c|c|c|}
\hline No. & Country & No. & Country & No. & Country \\
\hline 1 & Afghanistan & 7 & Greece & 2 & Papua New Guinea \\
\hline 1 & Albania & 2 & Guatemala & 2 & Paraguay \\
\hline 4 & Algeria & 1 & Guinea & 3 & Peru \\
\hline 2 & Angola & 1 & Guinea-Bissau & 18 & Philippines \\
\hline 17 & Argentina & 1 & Guyana & 22 & Poland \\
\hline 369 & Australia & 1 & Haiti & 2 & Portugal \\
\hline 30 & Austria & 2 & Honduras & 1 & Qatar \\
\hline 1 & Bahamas & 4 & Hong Kong & 4 & Romania \\
\hline 1 & Bahrain & 9 & Hungary & 93 & Russia \\
\hline 6 & Bangladesh & 2 & Iceland & 1 & Rwanda \\
\hline 3 & Barbados & 86 & India & 1 & St. Lucia \\
\hline 41 & Belgium & 9 & Indonesia & 1 & Sao Tome \& Principe \\
\hline 1 & Belize & 3 & Iran & 5 & Saudi Arabia \\
\hline 1 & Benin & 15 & Ireland & 2 & Senegal \\
\hline 3 & Bolivia & 25 & Israel & 1 & Seychelles \\
\hline 3 & Botswana & 50 & Italy & 1 & Sierra Leone \\
\hline 28 & Brazil & 1 & Ivory Coast & 6 & Singapore \\
\hline 2 & Brunei & 1 & Jamaica & 1 & Solomon Islands \\
\hline 6 & Bulgaria & 95 & Japan & 2 & Somalia \\
\hline 2 & Burkina Faso & 5 & Jordan & 14 & South Africa \\
\hline 2 & Burma & 29 & Kenya & 9 & South Korea \\
\hline 1 & Burundi & 1 & Laos & 21 & Spain \\
\hline 1 & Byelorussia & $i$ & Lebanon & 9 & Sri Lanka \\
\hline 1 & Cameroon & 1 & Lesotho & 3 & Sudan \\
\hline 213 & Canada & 1 & Libya & 1 & Suriname \\
\hline 2 & Cape Verde & 1 & Lithuania & 1 & Swaziland \\
\hline 2 & Central African Republic & 2 & Luxembourg & 41 & Sweden \\
\hline 1 & Chad & 1 & Madagascar & 69 & Switzerland \\
\hline 7 & Chile & 2 & Malawi & 1 & Syria \\
\hline 88 & China, People's Republic & 61 & Malaysia & 11 & Taiwan \\
\hline 4 & Colombia & 1 & Maldives & 5 & Tanzania \\
\hline 1 & Congo & 1 & Mali & 18 & Thailand \\
\hline 7 & Costa Rica & 4 & Malta & 1 & Togo \\
\hline 7 & Cuba & 2 & Mauritania & 3 & Trinidad/Tobago \\
\hline 2 & Cyprus & 1 & Mauritius & 1 & Tunisia \\
\hline 3 & Czechoslovakia & 6 & Mexico & 4 & Turkey \\
\hline 14 & Denmark & 2 & Monaco & 3 & Uganda \\
\hline 1 & Djibouti & 1 & Mongolia & 3 & Ukraine \\
\hline 1 & Dominican Republic & 1 & Morocco & 227 & United Kingdom \\
\hline 2 & Ecuador & 1 & Mozambique & 5109 & United States \\
\hline 14 & Egypt & 4 & Nepal & 3 & Uruguay \\
\hline 2 & El Salvador & 74 & Netherlands & 1 & Uzbek \\
\hline 3 & Estonia & 30 & New Zealand & 7 & Venezuela \\
\hline 5 & Ethiopia & 1 & Nicaragua & 3 & Vietnam \\
\hline 5 & Fiji & 5 & Niger & 1 & Western Samoa \\
\hline 11 & Finland & 14 & Nigeria & 2 & Yemen \\
\hline 87 & France & 1 & North Korea & 7 & Yugoslavia \\
\hline 1 & Gabon & 24 & Norway & 1 & Zaire \\
\hline 1 & Gambia & 7 & Pakistan & 2 & Zambia \\
\hline 145 & Germany & 1 & Panama & 4 & Zimbabwe \\
\hline 7 & Ghana & & & & \\
\hline
\end{tabular}

aAs defined in The Statesmen's Year-Book, John Paxton, ed., 1985. 


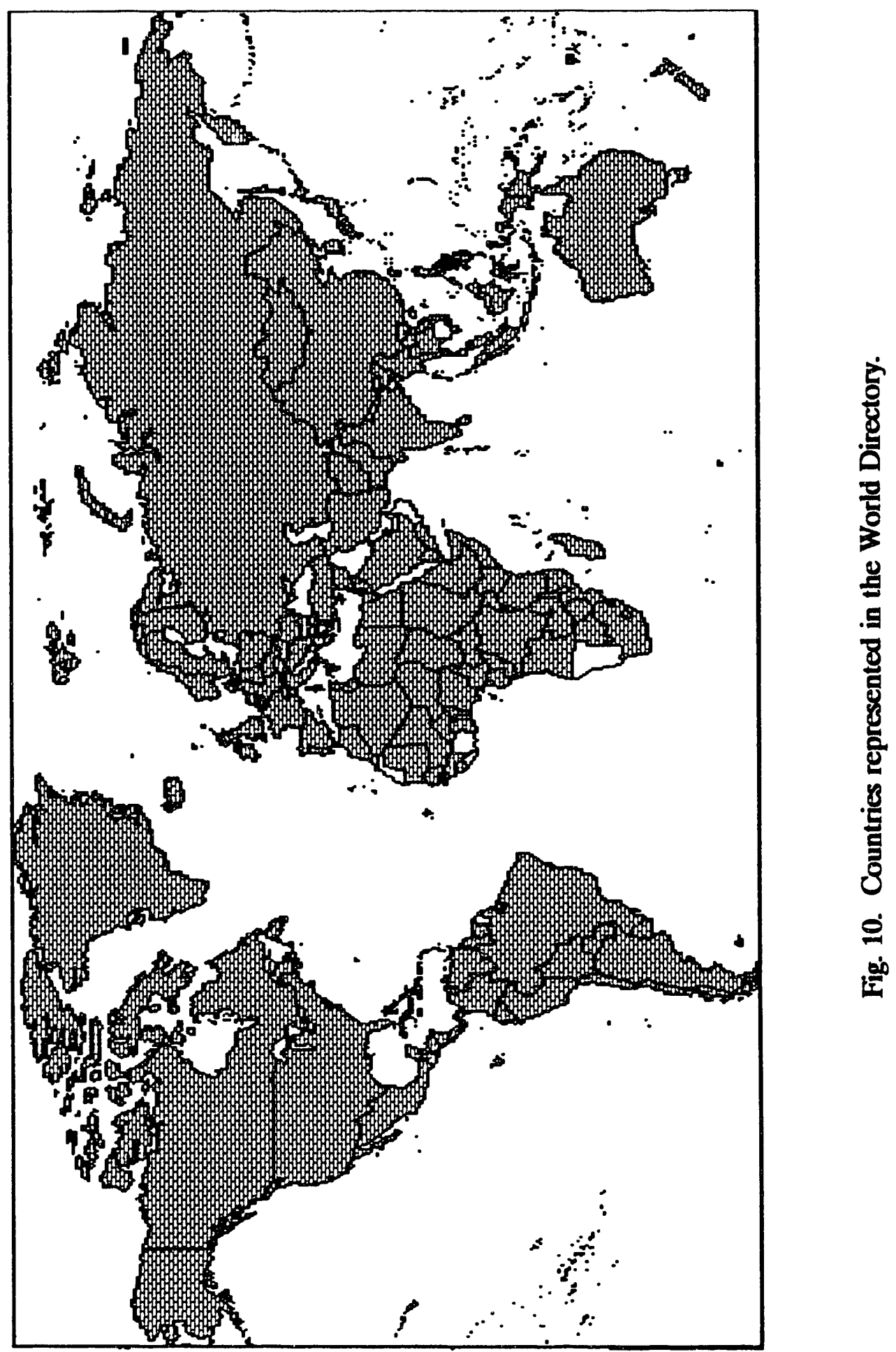




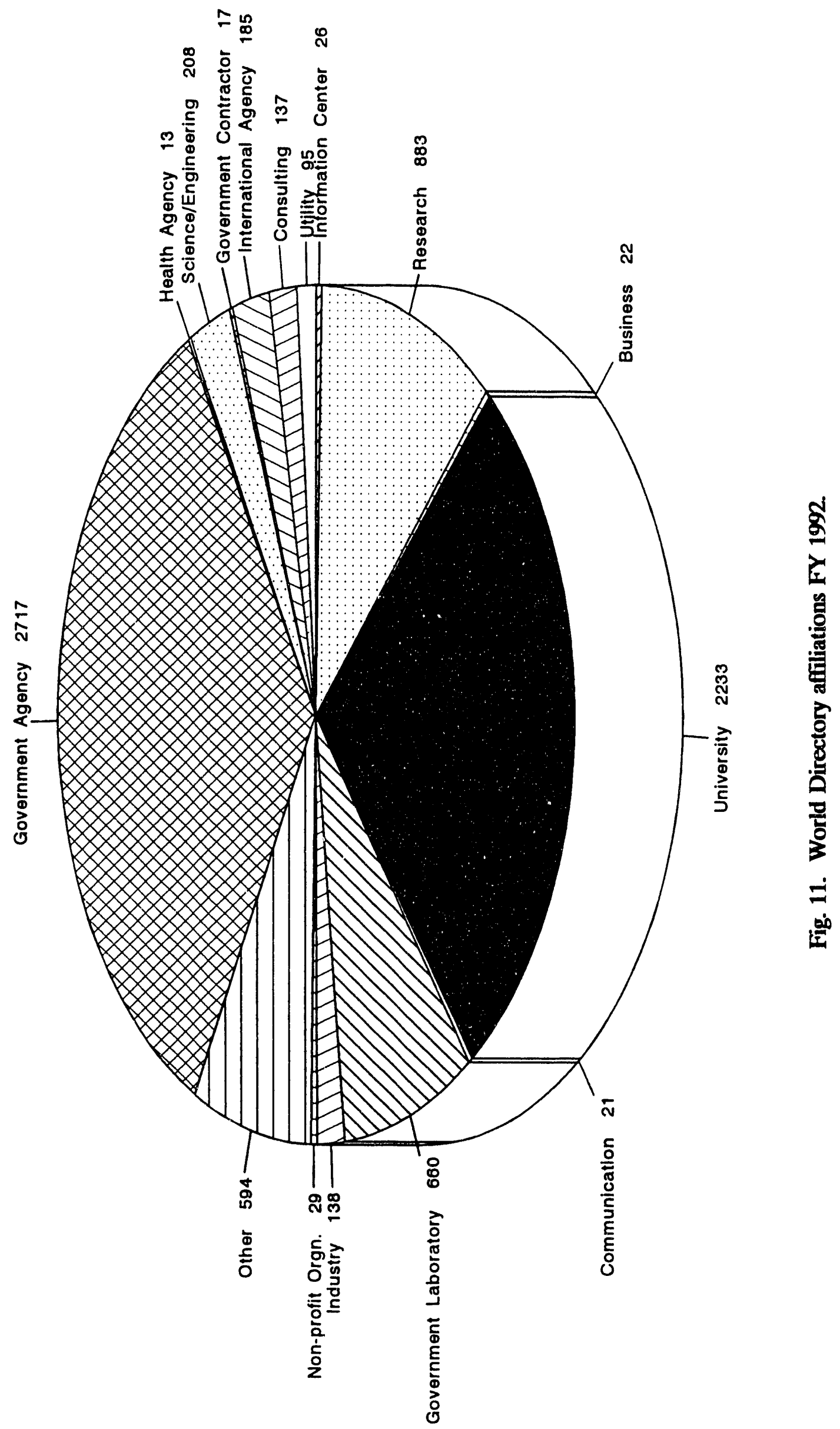




\section{Systems}

CDIAC relies heavily on computers to carry out our duties as an information center. Data acquisition, data validation, data distribution, data archival, maintaining customer data bases, and filling customer requests are but a few of the routine operations that require the use of various computer systems. Over the years we have developed a number of computer systems to assist us in our daily operations. The past year has been a busy period for computer systems development at CDIAC.

\section{CDIAC Computing System Network (CCSN)}

- In the past CDIAC has relied on ORNL hardware resources for performing data validation, distribution, and archival operations. With the trend to large, spatial data sets, CDIAC's computer related operating costs were rapidly increasing. By owning the computer tools needed for our operation, we can better control these costs. With this in mind, the CCSN was designed. We continue to make steady progress to free CDIAC from its past reliance on ORNL hardware resources and the associated increasing operating costs. Details of the CCSN follow:

The CCSN is comprised of a network of 12 high-speed Sun workstations, on-line disk capacity in excess of 8 gigabytes, an optical disk jukebox with an on-line capacity of 6.5 gigabytes and an infinite near-line capacity, a $1600 / 6250$ bpi $1 / 2^{n}$ tape drive, an $8 \mathrm{~mm}$ tape drive with a 5 gigabytes capacity, a $1 / 4^{n}$ tape drive, and a CD-ROM.

All of CDLAC's data holdings are stored on the CCSN.

All of CDIAC's data validation is done on the CCSN via the various installed SAS'm products (Base SAS, SAS Graph, SAS FSP, SAS Stat, and SAS AF), Fortran, and C.

All of CDIAC's data holdings are archived on the CCSN's optical jukebox.

All of CDIAC's NDP associated data are on-line.

The CCSN is capable of producing $1 / 2^{n}$ 9-track tapes of virtually any standard format. We are presently working on a user interface that will allow CDIAC staff to fill specialized NDP requests via the CCSN.

All of CDIAC's Geographical Information System (GIS) work is done on the CCSN via ESRI's ARC/INFO software suite.

Framemaker, a desktop publishing system, is available on the CCSN.

- Recent upgrades include the following:

Operating systems of all CDLAC workstations to SunOS 4.2 have been upgraded.

The windowing system of all CDIAC workstations to OpenWindows 3.0 has been upgraded.

Orders have been placed for hardware to upgrade the CDIAC server from a SPARCstation $1+$ to a SPARCstation 2. This upgrade should result in a $45 \%$ increase in CPU speed. In addition to the CPU upgrade, the CDIAC server will receive an additional 1.2 gigabytes of disk storage space. 
- ARC/INFO digitizing capability for the CDIAC network has been set up.

- ARC/INFO licenses for central ESD GIS workstation have been purchased.

All workstations have been upgraded to at least $16 \mathrm{MB}$ of RAM. This additional memory greatly increases the performance of the SAS products and of the ARC/INFO GIS software. Additional memory upgrades are planned.

A number of no-cost patches and upgrades have been applied to the somewhat problem-plagued rewritable optical jukebox. It appears that the early problems have been remedied. The optical jukebox has been steady for several months and is seeing heavy usage at this time.

\section{CDIAC Information System (CIS)}

- In addition to maintaining climate related data for distribution to the public, CDIAC also maintains internal data critical to the CDIAC's operation. These data include the names, addresses, order history, and so on for almost 7,000 customers. These systems comprise the CDIAC Information System.

The Request Response Record System (R3)/World Directory System has been completely re-written and combined into a single relational data structure in order to better accommodate the increasing number of customers and requests. The new system resides on a $33 \mathrm{MHz} 486$-based PC and utilizes a windowing user interface. We are very pleased with the new 486/windows-based system. Performance improvements are significant, and the additional capabilities are extremely useful. Perhaps the most useful of the new features is the ability to pull up customer profiles, including their order history, while conversing with them on the telephone. The slower performance of the old system made this cumbersome at best.

\section{CDLAC Anonymous File Transfer Protocol (FTP)}

- The CDIAC Computing System Network (CCSN) server has been set up as an anonymous FTP site. As such, all NDP data are freely accessible world-wide to customers with Internet access.

- Records are maintained which show what was accessed, by whom, and when. Our goal is to automatically integrate this information into the Request Response Record. At present, the information is entered into $R^{3}$ manually, the same as any other request.

- Automated routines have been set up to electronically mail all anonymous FTP users a brief description of CDIAC and the services we offer.

- FTP service is seeing considerable usage and is a definite success; the NDP/CMP Distribution section of this report shows statistics on the requests made and filled.

- 145 customers accessed CDIAC's data through the anonymous FTP area in FY 1992. 


\section{Russian/English Translation System}

- CDIAC had the fortune of hosting Carolina Ravina from Obninsk, Russia, as part of the separate Quantitative Links Support project (see p. 73). Carolina used her skills as a linguist to translate a number of documents into Russian. This necessitated the development of a computer system capable of supporting Cyrillic text display and printing functions. We developed a $386 / 33 \mathrm{MHz}$ PC-based system supporting an HP laser printer and color scanner. The system runs Globalink's translation software and the WordPerfect Russian module, and supports document scanning via Inovatic's Readstar software.

It is our hope that Ms. Ravina will continue to use her skills as a linguist to translate documents of scientific content in order to assist Russian/U.S. collaborative research. We have offered support for this activity by offering to loan to the All-Russian Research Institute of Hydrometeorological Information the PC-based translation system used by Ms. Ravina while at CDIAC. We are working at present to secure commodity classification from the Department of Commerce for the system. Once the proper documentation has been secured, the system will be sent to Obninsk. 


\section{Communications}

- CDIAC produced 3000 copies of Trends '91: A Compendium of Data on Global Change. Distribution of this report is to select researchers, policy makers, libraries, and administrators in government, corporate, and nonprofit settings. Additional distribution will be made to those individuals requesting copies of the report. Promotion of the availability of this report has been made through CDLAC Communications, a media alert issued by the Public Relations Department of Oak Ridge National Laboratory, a factsheet produced and distributed by CDIAC.

- CDIAC producted 8500 copies of a 70-page summary document, Trends '91: A Compendium of Data on Globai Change-Highlights, which has been distributed to all persons and organizations listed in CDIAC's World Directory of Researchers and Policymakers (more than 7000 through June 1992). Trends Highlights was designed to be a self-contained bulk-mail item for domestic distribution.

- The sixteenth issue of CDLAC Communications was published as the Winter 1992 issue. This 32-page issue reflects a re-design in the format and style of the newsletter, including its production in 2 colors. This issue was produced as a special 10th anniversary edition, commemorating CDIAC's 10-year history as part of the Department of Energy's Global Change Research Program. One other major design element in this newsletter facilitates the domestic distribution of CDLAC Communications as a selfcontained, bulk-mailing for domestic (U.S.) distribution. Foreign distribution remains the same as airmail, printed material. CDIAC also produced the summer 1992 issue of CDLAC Communications.

- CDIAC staff, in conjunction with DOE Program Managers and principal investigators produced 8 issues in the DOE Research Summary series (formerly the Research Project of the Month series):

No. 12 November 1991 The MINK Study: The Regional Effects of Changing $\mathrm{CO}_{2}$ and Climate. Michael Scott

No. 13 December 1991 Doubling $\mathrm{CO}_{2}$ Triples Growth Rate of Sour Orange Trees. Sherwood B. Idso and Bruce A. Kimball

No. 14 January 1992 Recent Rise of the Nighttime Temperatures in the Northern Hemisphere. George Kukla and Thomas R. Karl

No. 15 February 1992 Aerosols, Clouds, and Climate. James Hudson

No. 16 April 1992 Testing for Trend in the Global Temperature Data. Wayne A. Woodward and Henry L. Gray

No. 17 June 1992 Intercomparison and Interpretation of Surface Energy Fluxes in Atmospheric General Circulation Models. David A. Randall and Robert D. Cess.

No. 18 August 1992 Estimating Biomass Change of Tropical Forests. Sandra Brown, Louis R. Iverson, Anantha Prasad, and Ariel E. Lugo.

No. 19 September 1992 The Single-Operator Multiparameter Metabolic Analyzer for Total Carbon Dioxide with Coulometric Detection. Kenneth M. Johnson and Douglas W. R. Wallace.

The popularity of the DOE Research Summary series continues to grow. Bill Purvis of the National Coal Association asked CDIAC for permission to reprint roughly 1000 copies of DOE Research Summary No. 14, Recent Rise of the Nighttime Temperatures in the Northern Hemisphere, for distribution to its members and affiliates. 
- In conjunction with the distribution of the Winter 1992 issue of CDLAC Communications was the production and distribution of a CDIAC Survey. This survey was developed to provide insight on the information and data management capabilities and needs of CDIAC's user community. The survey will also provide input from this user community on their opinions on CDLAC Communications and Trends. All entries in CDIAC's World Directory and those persons requesting CDIAC's numeric data packages and reports (but are not in the directory) received this survey.

- CDIAC received a request from Saunders College Publishing (a subsidiary of Harcourt Brace and Jovanich College Publishers, a contact niade at the 1991 American Libraries Association Annual Meeting in Atlanta) to reproduce the graph of atmospheric concentrations of $\mathrm{CO}_{2}$ at Mauna Loa, as depicted in Trends '91. The form for properly citing the original work of Dave Keeling was included. This illustration is to be used in an upcoming text book, The Environment.

- CDIAC prepared a factsheet, Technical Assistance for Developing Regions, to better respond to inquiries seeking financial assistance for various third-world funding needs. This new factsheet briefly describes the availability of support from the Global Environmental Facility and the Rockefeller Foundation.

- Through its affiliation with the Interagency Working Group for Data Management on Global Change (Cushman, Kanciruk, and Stoss), CDIAC received information about WAIS software, a wide area network accessible through various eMAIL services, including the INTERNET, the Special Issues Document for creating a Global Change Data and Information Service, the development of a global change lexicon or Thesaurus, and development of a bibliographic data base as a file in the Global Change Master Directory.

- CDIAC began a subscription service to Environmental Information Network, Inc's. Global Warming Network. CDIAC opted not to take the online access, but to receive the daily FAX services for global warming and ozone depletion. Each service provides extracts of relevant information gathered from scores of newspapers, newsletters, and other general interest/business publications.

n Through the University of Tennessee's Energy, Environment, and Resources Center; CDIAC provided the University of Tennessee Libraries with a complete set of publications and information products provided by CDIAC.

- CDIAC revised its Catalog of Data Bases and Reports and developed a new catalog of numeric data and computer model packages.

- Fred Stoss gave a 45-minute discussion about CDIAC for the April meeting of Interagency Working Group on Data Management for Global Change Library/Information Subgroup (of which Stoss is a member). In addition he was asked to review his effort in working with ORNL's Center for Global Environmental Studies in developing an organizational chart of the U.S. Executive branch activities in global change research and policy.

- CDLAC was represented at the October meeting of Working Group VIII of the U.S.-U.S.S.R. bilateral agreement on protection of the environment, which described various U.S.-Soviet research and policy initiatives, exchanges of resources and personnel, and future needs of the working group. A major theme of the workshop was the continuation of existing bilateral agreements, and in light of the rapidly changing political climate in the Soviet Union, to seek these continuances with a delicate yet forceful action. Continuing these agreements was seen by both U.S. and Russian attendees as critical in meeting the growing technical needs of climate change research. A demonstration of the Globalink Russian-to-English translation software was made. 
- NOAA's National Geophysical Data Center requested permission to distribute portions of NDP-17 (Major World Ecosystem Complexes).

- Fred Stoss gave a presentation on the topic of Environmental Trends to the Southern Appalachian Chapter of the Special Libraries Association in May.

- CDIAC obtained a copy of the NASA TOMS Ozone Image Data, 1978.91 CD-ROM. The CD provides color images (north and south polar and world) of stratospheric ozone. Images can be obtained for daily or monthly averages. The disk has been placed in the ORNL/ESD Library.

- CDIAC provided David Reichle (Associate Director at ORNL), Bob Van Hook ORNL/ESD Director, and Tom Gross, U.S. DOE GCRP Program Manager, with a series of color photographs depicting various aspects of CDIAC operations and products. The photos and captions will be used to assist their general talks and presentations about ORNL/ESD capabilities and achievements.

- CDIAC received 250 copies of DOE's Energy Education booklet from Paula Altman (National Energy Information Agency in the Energy Information Administration). CDIAC uses these booklets to help answer inquiries from primary and secondary teachers, students, and others seeking education-oriented information.

- CDIAC extended the internal (ORNL) distribution of all CDIAC publications to include all division directors and associate directors.

- $\mathrm{CDIAC}$ also published a variety of reports, newsletters, and other materials, including a revision of the CDIAC brochure, a revision of CDIAC's Catalog of Data Bases and Reports (ORNL/CDLAC-34/R2), a third reprinting of the third edition of Glossary: Carbon Dioxide and Climate (ORNL/CDLAC-39), Carbon Dioxide Information Analysis Center: FY 1991 Activities (ORNL/CDIAC-48), and Global Change Numeric Data, CDIAC's (and apparently ORNL's) first CD-ROM.

- CDIAC has reviewed various materials about the proposed U.S. GPO Gateway/WINDO, which is a comprehensive data management structure to be furnished by the GPO and which will provide an active interface to all U.S government data bases.

- Trends ' 90 has been cited in professional papers in the following journals: the Bulletin of the American Meteorological Society, Environmental Conservation, Science, Geochimica et Cosmochimica Acta, Doklady Akademii Nauk SSSR, Climatic Change, Marine Chemistry, Environmental Science and Technology, and Nature (representing a very handsome set of professional literature in which to be cited).

In addition to these journal citations, Trends ' 90 and Trends ' 91 have been cited in many reports and position papers not covered by the Institute for Scientific Information. The Trends citations include materials prepared by the World Resources Institute, Gale Research Company, the United Nations Environment Programme, the International Geosphere-Biosphere Program, and World Meteorological Organization.

- Gregg Marland's $\mathrm{CO}_{2}$ emissions data was used in compiling the top ten carbon dioxide emitters in the world, which appeared in a delightful book. The Top 10 of Everything, published in the United Kingdom by Rykehurst House.

- Information about CDIAC and its publications has been found in various newsletters: Global Warming Network Online Today, Inside Energy, Global Environmental Change Report, Energy Daily, and Environment Week. 
- At the annual meeting of the Special Libraries Association Fred Stoss participated on a 6-member panel examining environmental trends, and on a 2-member panel discussing the proposed National Library for the Environment. 300 CDIAC brochures and factsheets, 100 CDLAC Communications, and 100 Trends Highlights were distributed at this conference.

- Beyond CDLAC but critical to our needs...

Gay Marie Logsdon, editor for ORNL's Environmental Science Division, and Cheryl Buford, from Advance Publications Technologies (both in ORNL's Publications Division), received division acknowledgement for their work on Trends ' 91 .

\section{Special Visits}

- CDIAC hosted a meeting with Tadashi Matsunaga (Professor of Biotechnology at the Tokyo University of Agriculture) and Hiroyuki Takano (New Fields Research Laboratory). These individuals were in Oak Ridge to participate in a series of meetings and seminars related to alternative sources of energy.

- Janet Wright, head of the DC Reference Center of the National Agricultural Library, paid an extended visit to CDIAC to discuss various data and information management activities at CDIAC. The National Agricultural Library is seriously contemplating the expansion of their services to include the development of their own specialized information analysis centers. CDIAC might become the model for future NAL IACs!

- CDIAC hosted an extensive visit and review by the National Research Council and CODATA for their assessment criteria for data base integration.

\section{CDIAC Goes to Conferences}

- Former CDIAC Directors Michael P. Farrell and Paul Knaciruk attended the 1992 United Nations Conference on Environment and Development (UNCED, the Earth Summit) in Rio de Janeiro taking along 500 copies of CDLAC Communications CDIAC's brochure, and fact sheets about CDIAC;

- Russ Vose attended the International COADS Workshop in Boulder, Colorado, January 1992.

- Russ Vose attended the International Temperature Workshop in Boulder, Colorado, January 1992.

- Russ Vose attended the Association of American Geographers annual meeting in San Diego, California, April 1992.

- Tammy White attended the 12th Annual Environmental Systems Research Institute's ARC/INFO User Conference in Palm Springs, California, June 1992.

- Fred Stoss attended the Annual Conference of the Special Libraries Association in San Francisco, California, June 1992.

- Materials about CDIAC were distributed at the following conferences:

TJFR Executive Media Institute Environmental Journalism Conference (June, New York)

United Nations Conference on the Environment and Development (June, Rio de Janeiro)

Annual North American Natural Resources Librarians/Information Specialist Conference (September, Boulder, Colorado) 


\section{CDIAC Assists Government Environmental Managers}

- Beginning in late September the U.S. Office of Personal Management conducted an extensive 1 week training program, Environmental Policy Issues, for senior government agency managers. CDLAC material were displayed at the meeting, where global change issues were used as simulation scenarios for developing policy initiatives. One attendee utilized CDIAC's resources to help with the information assessment of baseline data. Her co-attendees were impressed with CDIAC's ability to respond so thoroughly, effectively, and quickly.

\section{Expanding CDIAC's Library Network}

- Information about CDIAC, sample copies of newsletters, and surplus stocks of reports were distributed to the following international libraries and library associations:

American Libraries Association, Association of College and Research Libraries (Chicago) American Libraries Association, Public Library Association Canadian Library Association

Environmental Protection Agency Libraries (Headquarter and Regional Offices)

Green Library (Berkeley, CA)

Green Library (Riga, Latvia)

Green Library (Warsaw, Poland)

Inter-American Association of Agricultural Librarians and Documentalists (Coronado, Costa Rica) International Federation of Library Associations and Institutions (The Hague, The Netherlands) International Association of Technological University Libraries (Enschede, The Netherlands) Latin American Library Enhancement Project (Lawrence, KS)

United Nations Environment Programme, INFOTERRA National Focal Points

\section{CDIAC Directory Updates}

- Entries for CDIAC were updated or revised in the following directories:

American Water Works Association Youth Education Survey

Energy Education Resources (Kindergarten through 12th Grade)

Gale Research Directory of Special Libraries and Information Centers

Research Data Management in the Ecological Sciences

World Environmental Research Directory

National Agriculture Library's revised "Global Change Information Packet" 


\section{Collaboratıve Efforts with China}

- DOE 'Technical Report on Chinese Climate Data is Completed

Climatic Data Bases of the People's Republic of China, 1841-1988

(Report number yet to be determined).

The draft version of this technical report (prepared FY 1991) was improved and finalized. It describes data from 296 stations, contributed by the Chinese Academy of Sciences (CAS), which are organized as five data sets: (1) a 60-station data set containing monthly measurements of barometric pressure, surface air temperature, precipitation amount, relative humidity, sunshine duration, cloud amount, wind direction and speed, and number of days with snow cover, (2) a 205-station data set containing monthly mean temperature and monthly precipitation totals, (3) a 40-station subset of the 205-station data set containing monthly mean maximum and minimum temperatures and monthly extreme maximum and minimum temperatures, (4) a 180-station data set containing daily precipitation totals, and (5) a 147-station data set containing ten-day precipitation totals. Sixteen stations from these data sets (13 from the 60-station, 3 from the 205-station) have temperature and/or precipitation records which begin prior to 1900 , whereas the remaining stations began observing in the early to mid-1900s. Records from most stations extend through 1988.

The report includes descriptions of the data sets, station inventories and histories, maps of the five station networks, and a quality assessment of the data. These data sets represent the most comprehensive, long-term instrumental Chinese climate data presently available.

- Tom Boden attended the U. S. DOE/PRC CAS meeting in Albany in October 1992 and presented a talk on what is needed to fully document the PRC instrumental and proxy data records. He is now awaiting input from Professors Zhang Peiyuan and Wei-Chyung Wang to fully document the proxy climate data received from PRC.

- Sent Professor Fu the GFDL GCM output archived at CDIAC including the version that offers 10-year monthly averages that he requested at the meeting, however, after reviewing the data Fu indicated he needed a later version of the model that provides 10 -year monthly averages. Contacted Richard Wetherald and subsequently Roy Jenne to obtain this later version. This latter version was provided to CDIAC by Roy Jenne at NCAR.

- Provided PRC proxy data to Ray Bradley, Gregg Garfin, and David Portman.

- The NDP that documents the PRC 60-and-205 station network climate records (see NDP section) was completed. 


\section{Numeric Data and Computer Model Packaging}

\section{Requests for NDPs and CMPs}

- CDIAC staff tilled 910 requests from 48 countries for 1,684 copies of numeric data packages (NDPs) and 132 copies of the PC-version of the IEA/ORAU Long-Term Global Energy $\mathrm{CO}_{2}$ Model. In filling requests for machine-readable files, 173 9-track magnetic tapes and 1,120 floppy diskettes were generated. This represents the largest number of data and computer model requests ever filled in the 11-yeir history of CDIAC and represents a nearly 50\% increase over FY 1991. More requests were filled during the second half of FY 1992 than were filled in all of FY 1991. Activities related to NDPs and CMPs are found in Figss. 12, 13 and 14. Table 7 provides a list of countries to which NDPs and CMPs were sent, while Table 8 provides a distribution inventory of all NDPs and CMPs from 1985 through FY 1992.

- Tommy Nelson and Dave Sill established an anonymous FTP area in February on CDIAC's SUN SPARC wor'station server. This area is accessible through the Internet network and contains all of CDIAC's NDP and CMP files. During FY 1992, 145 individuals accessed CDLAC's new anonymous FTP area and retrieved over 10,000 individual files. Requesters are required to enter a valid Internet or Bitnet address to access the system, which permits CDLAC to maintain appropriate request records, send follow-up letters offering complete written documentatiol, and notifying past recipients of data updates or revisions.

- CDIAC staff provided those scientists who have contributed data or models to CDIAC for dissemination as NDPs and CMPs with lists of the individua!s that have requested and received their data from CDIAC.

- CDIAC produced 200 copies of its first CD-ROM and announced its availability in the March issue of CDLAC. Communications; by May all 200 copies had been distributed. Another CD-ROM containing all of CDLAC's NDPs and CMPs is planned for FY 1993 and will actually be a two-volume set because of the size of some of our recent NDPs.

\section{Updated NDPs}

- Documentation (NDP-001/R3) was updated that presents the atmospheric $\mathrm{CO}_{2}$ concentration records from Mauna Loa compiled by Dave Keeling and his colleagues at Scripps Institution of Oceanography. This update will offer monthly and annual atmospheric $\mathrm{CO}_{2}$ mixing ratios through 1992 . The last version of the NDP presented data through 1988.

- Documentation (NDP-005/R2) was upaated that presents the NOAA/CMDL atmospheric $\mathrm{CO}_{2}$ concentration records from their flask and in situ sampling networks. This update will offer daily and weekly measurements for approximately 30 globally distributed sites through 1990 . The last version of the NDP presented data through 1988.

- Documentation (NDP-008/R3) and data files were updated for Jim Angell's global, hemispheric, and zonal stratospheric and tropospheric temperature anomalies. The update will present seasonal and annual aver ages through 1992.

- Documentation (NDP-010/R1) was updated that presents the atmospheric $\mathrm{CO}_{2}$ concentration records from Cape Grim, Tasmania compiled, by Dave Beardsmore and Graeme Pearman at CSIRO. This update will offer daily, monthly, and annual atmospheric $\mathrm{CO}_{2}$ mixing ratios through 1991 . The last version of the NDP presented data only through 1383. 


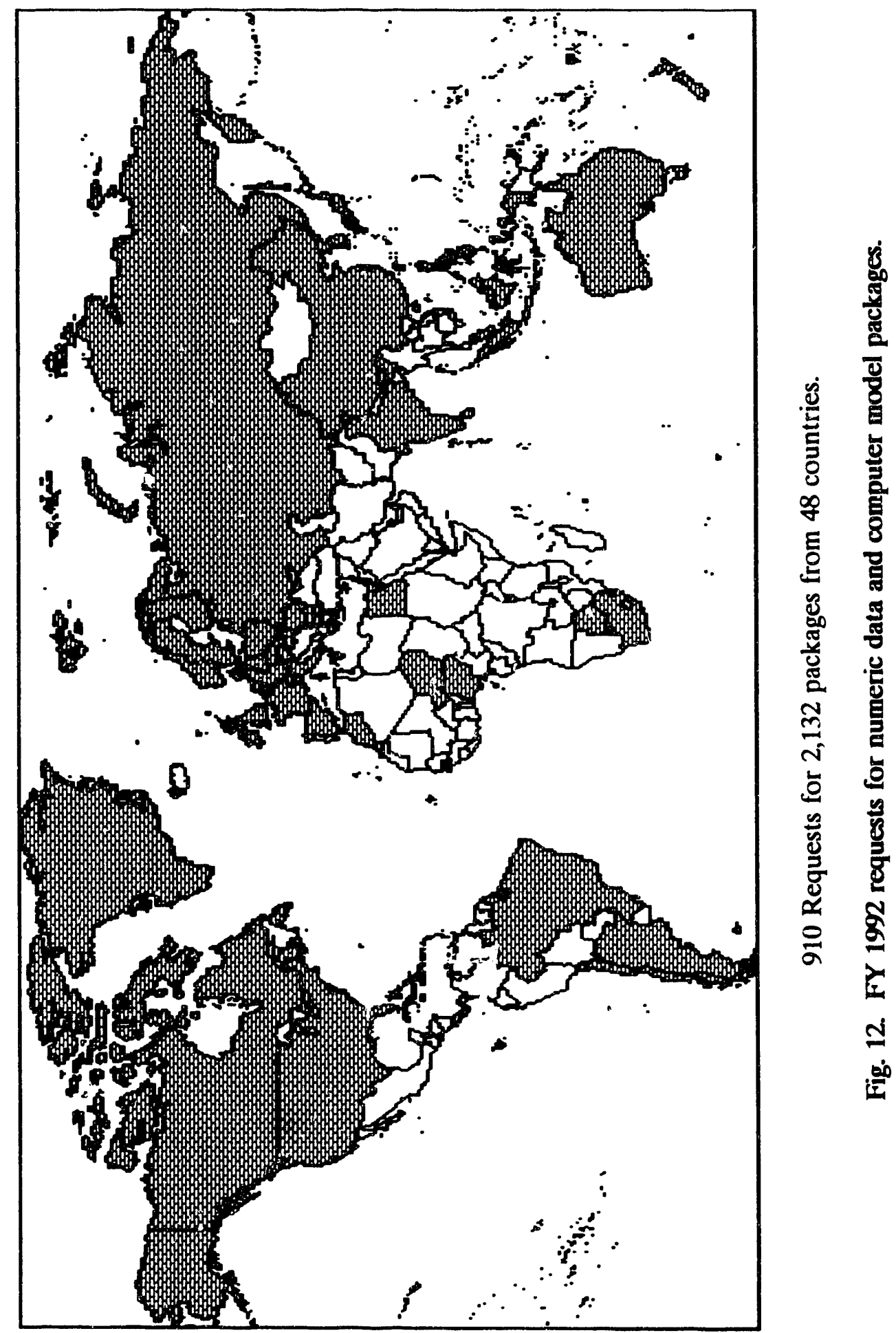




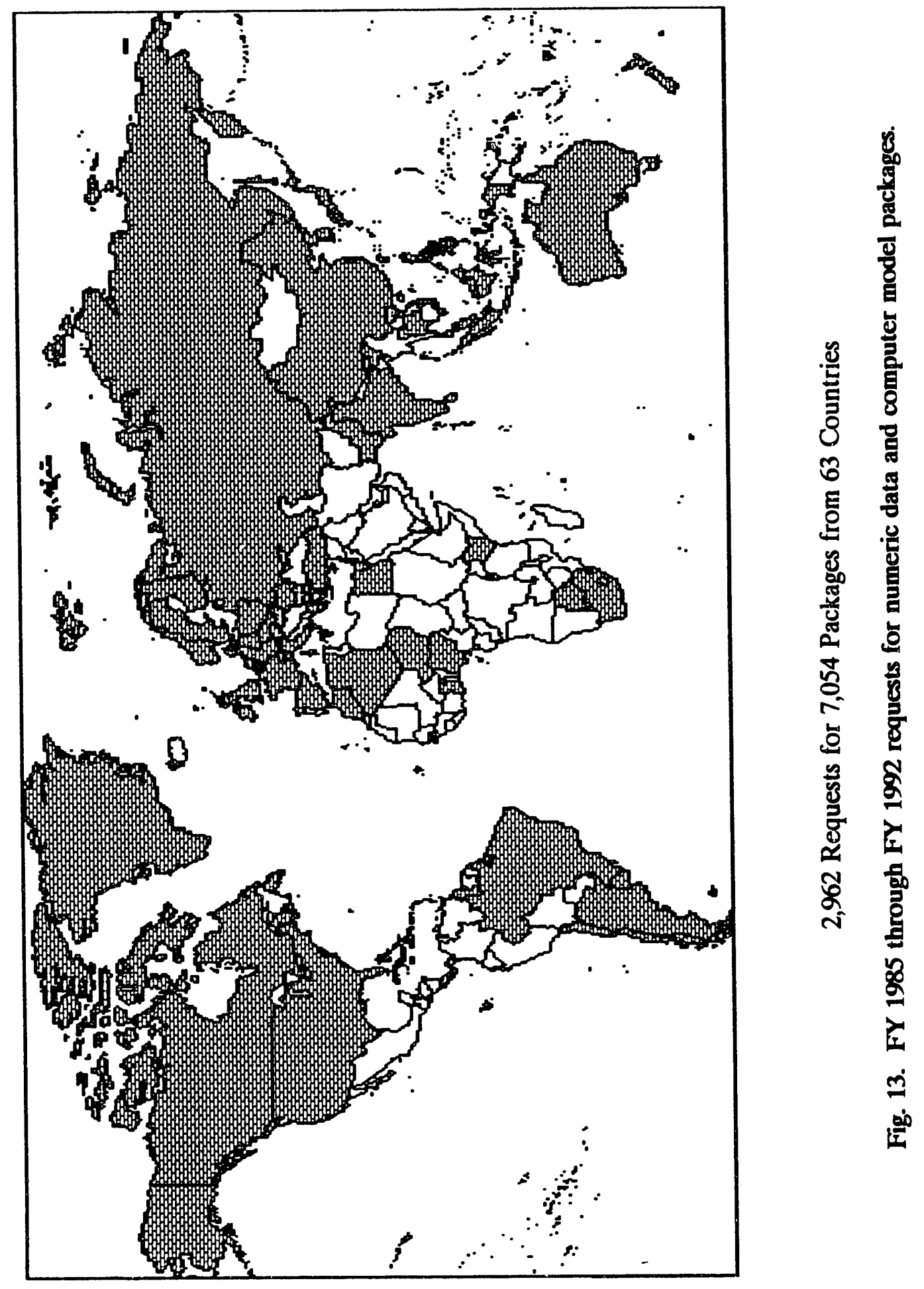




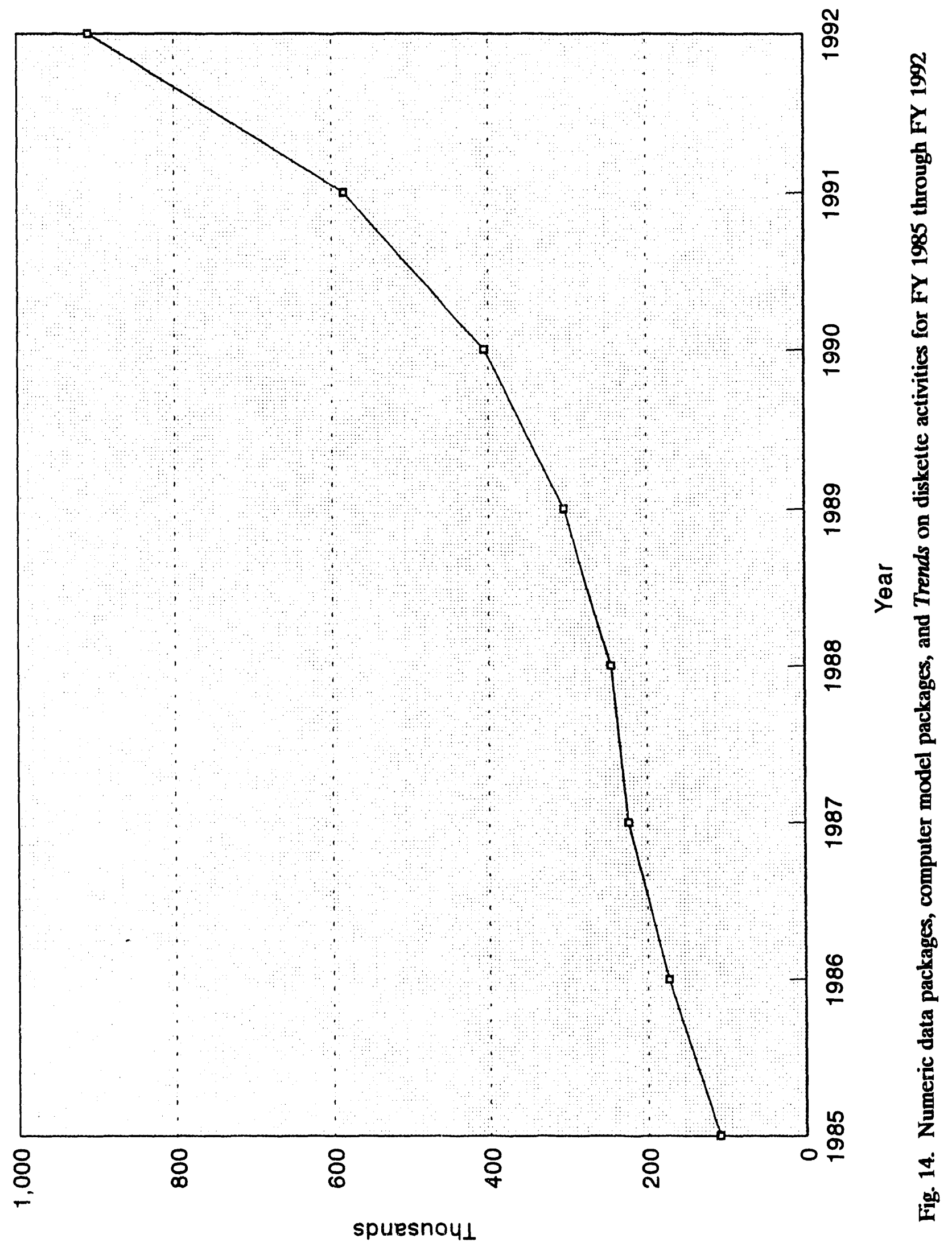


- CDIAC staff participated in attempts to make arrangements to improve Zinke et al.'s organic soil carbon and nitrogen profile data base (NDP-018). This data base, which contains data from over 3500 soil profiles taken around the world, is one of the best and most frequently used data bases for studies attempting to determine the amount of carbon sequestered in the soil. The previous NDP contains only profiles sampled to a depth of $1 \mathrm{~m}$ and for select variables. Inclusion of other profiles (approximately 1200 ), addition of cation measurements, and assignment of soil taxonomy classifications to each soil profile would greatly enhance the utility of the data base (e.g., studies of the soil carbon dynamics in tropical forests). The cation determinations, along with other measurements, were made shortly after the profile was collected but reside on punch cards stored at the University of California, Berkeley (UCB). This fact, along with the fact that Paul Zinke, the person most capable person of assigning the soil taxonomy classifications, retired last year from UC-B prompted the ORNL Environmental Sciences Division's Mac Post to propose this collaborative recovery and enhancement effort. Mac collaborated on the original compilation of this project. CDIAC's role in this project might be to assist UC-B in recovering the data from cards, converting the information into another digital form, and then building and documenting the enhanced data set. Mac Post has communicated with Zinke and Al Stangenberger to promote the idea and determine what efforts are needed. Initial response has been very favorable and a possible UC-B/Zinke/CDIAC/ORNL Carbon Cycle group collaborative effort seems likely.

- NDP-020/R1 An Updated Global Grid Point Surface Air Temperature Anomaly Data Set: 1851-1990. This revised NDP presents land-based monthly surface air temperature anomalies (departures from a 1951-1970 reference period mean) on a $5^{\circ}$ latitude by $10^{\circ}$ longitude global grid. Monthly surface air temperature anomalies (departures from a 1957-1975 reference period mean) for the Antarctic (grid points from $65^{\circ}$ to $85^{\circ} \mathrm{S}$ ) are presented in a separate data set. While the period of record extends from 1851 to 1990 , few grid point locations have contributed data for the entire period of record.

- Began preparation of NDP-030/R4 Global, Regional, and National $\mathrm{CO}_{2}$ Emission Estimates from Fossil Fuel Buming, Cement Production, and Gas Flaring: 1950-1990. This update will be completed in FY 1993 and contains Gregg Marland and Tom Boden's global, regional, and national $\mathrm{CO}_{2}$ emission estimates through 1990. Boden received the UN energy statistics in April of 1992, checked the statistics, generated the emission estimates during the summer, and is now updating the documentation for one of CDIAC's most frequently requested data bases (484 requests for the three previous versions of the data base to date).

\section{New NDPs}

- Tom Boden completed NDP-033 (Atmospheric $\mathrm{CO}_{2}$ Concentrations Derived from Flask Samples Collected at U.S.S.R.-Operated Sampling Sites) which contains atmospheric $\mathrm{CO}_{2}$ concentrations from the U.S.S.R. flask sampling programs at Teriberka Station, Ocean Station Charlie, Bering Island, and Kotelny Island. The availability of this NDP was announced in the recent CDLAC Communications. Given the disbandment of the U.S.S.R., the completion of this NDP was particularly timely.

- NDP-037 Carbon Dioxide Enrichment: Data on the Response of Cotton to Varying $\mathrm{CO}_{2}$, Irrigation, and Nitrogen. This NDP presents results from field $\mathrm{CO}_{2}$-enrichment experiments conducted over five consecutive growing seasons, 1983-1987. These results comprise data concerning the effects of continuous $\mathrm{CO}_{2}$ enrichment on the growth of cotton under optimal and limiting levels of water and nitrogen. Unlike many prior $\mathrm{CO}_{2}$ enrichment experiments in growth chambers or greenhouses, these studies were conducted on field-planted cotton at close-to-natural conditions using the open-top chamber approach. Measurements were made on a variety of crop response variables at intervals during the growing season and upon crop harvest. The initial experiment examined the effects of varying $\mathrm{CO}_{2}$ concentration only. In the following two seasons, the interactive effects of $\mathrm{CO}_{2}$ concentration and water availability were studied. In the final two seasons, the effects of the three-way interaction between $\mathrm{CO}_{2}$ concentration, water availability, and nitrogen fertility were investigated. 
The data comprise three types of information: identification variables (such as year, institution and site codes, and treatment regimens), intermediate growth measurements (such as plant height, leaf area index, number of flowers, and dry weight of leaves) taken at various times during the growing season, and crop harvest results (such as lint yield, seed yield, and total aboveground dry biomass). The data will be useful for elucidating the possible future effects on crop production of a doubling of global atmospheric $\mathrm{CO}_{2}$ concentrations and for validating crop growth models designed to predict the effects of elevated $\mathrm{CO}_{2}$ on cotton growth. The data show that, for all five growing seasons and all experimental treatments (water-stressed or well-watered, low-nitrogen or high-nitrogen), cotton yields and plant dry weights were increased significantly by $\mathrm{CO}_{2}$ enrichment.

- NDP-039 Two Long-Term Instrumental Climatic Data Bases of the People's Republic of China. This NDP represents data from two long-term instrumental data bases containing meteorological observations from the People's Republic of China (PRC). This document represents the first information product resulting from the August 1987 joint research agreement signed by DOE and the PRC Chinese Academy of Sciences. Records from 265 stations are represented in this publication with some data sets going back to before 1900 .

- NDP-041, The Global Historical Climatology Network: Long-Term Monthly Temperature, Precipitation, Sea Level Pressure, and Station Pressure Data completed as part of the separate Quantitative Links support project (see p. 73). The NDP contains one of the largest and most comprehensive data sets that can be applied to the study of global climate change. The availability of the NDP was announced in the summer 1992 CDLAC Communications.

- NDP-042 United States Historical Climatology Network Daily Temperature and Precipitation Data. In addition to the intermediate growth and crop harvest data included in the NDP, data are also available for many other parameters, including daily weather, soil profile properties (e.g., initial soil moisture and nitrogen conditions), and irrigation and fertilizer treatments. These data are described in detail and presented in their entirety (in hard copy form) in a supplemental text provided as part of the documentation and may also be obtained in digitized form upon request.

- NDP-043A A Coastal Hazards Data Base for the U.S. East Coast. This NDP describes the contents of a digital data base that may be used by raster or vector geographic information systems (GIS) and nonGIS data bases to access the risks of coastlines to erosion or sea level rise. Each U.S. East Coast grid cell contains data on geology, geomorphology, elevation, wave heights, tidal ranges, shoreline displacement (erosion), and sea-level trends. Documentation for this NDP contains information on the methods used in calculating each variable; detailed descriptions of file contents and formats; and a discussion of the sources, restrictions, and limitations of the data.

\section{NDPs In Progress}

- NDP-044 Surface Water and Atmospheric Carbon Dioxide and Nitrous Oxide Observations by Shipboard Automated Gas Chromatography: Results from Expeditions between 1977 and 1990.

This NDP will present the results of surface water and atmospheric $\mathrm{CO}_{2}$ and $\mathrm{N}_{2} \mathrm{O}$ measurements carried out by shipboard gas chromatography over the period 1977-1990. These data comprise measurements taken from 11 different oceanic surveys for a total of 41 expedition legs.

The measurements were made by an automated high-precision shipboard gas chromatographic system developed by R. F. Weiss during the late 1970 s and used extensively over the intervening years. This instrument measures carbon dioxide by flame ionization after quantitative reaction to methane in a stream of hydrogen. Nitrous oxide is measured by a separate electron capture detector. The chromatographic system measures 196 dry-gas samples a day, divided equally among the atmosphere, gas equilibrated with surface water, a low-range gas standard, and a high-range gas standard. 
These data constitute one of the most extensive records available of $\mathrm{CO}_{2}$ and particularly $\mathrm{N}_{2} \mathrm{O}$ concentrations in marine air and surface seawater. The data will be valuable in modeling applications dealing with the ocean's role in the global cycles of carbon and nitrogen, in studies of ocean-atmosphere dynamics and in studies evaluating other methodologies for determining $\mathrm{pCO}_{2}$.

- NDP-046 Carbor-13 Isotopic Abundance and Concentration of Atmospheric Methane for Background Air in the Southern and Nivthern Hemispheres from 1978 to 1989.

This NDP will present atmospheric concentrations and $8^{13} \mathrm{C}$ values of $\mathrm{CH}_{4}$ from background air collected in 24 rural or marine sites, 15 in the Northern Hemisphere and 8 in the Southern Hemisphere, over the period 1978-1989.

The information provided by these data on the isotopic composition of atmospheric $\mathrm{CH}_{4}$ in each hemisphere will be a very useful aid in estimating the fluxes of the various natural and anthropogenic sources and in elucidating the causes of the seasonal cycle and the interhemispheric gradient of atmospheric $\mathrm{CH}_{4}$.

\section{- Trends '93}

Preliminary work was begun on the document Trends '93: A Compendium of Data on Global Change. This included exploring new graphics methods and identifying new data for inclusion in Trends. A style change will be implemented to reduce the redundancies of repeating methods and reference sections.

\section{Identification of Methane-Related Data Bases}

- During the spring and summer of 1992 , work was done to prepare an inventory of data bases related to the atmospheric methane cycle. This inventory began as an outcome of attendance at the NATO Advanced Research Workshop on The Aimospheric Methane Cycle, which was held October 6-11, 1991 at Timberline Lodge on Mt. Hood near Portland, Oregon.

- As part of the effort to identify existing methane-related data bases, two survey questionnaires were sent to a variety of individuals engaged in some aspect of methane research. As a result of this search, 195 data bases were identified. For each data base, an attempt was made to ascertain (and record) information describing the data base and defining the period of record, spatial coverage, principal investigators, source and availability, and any references that use or describe the data base.

- As an outcome of this project, we hope to publish the results of our inventory in some appropriate forum, preferably the open literature. In addition, the project has already led to the acquisition of a number of data sets to be archived by CDIAC and potentially offered as NDPs or summarized in Trends '93.

\section{Analysis of the Jones Gridded Surface Air Temperature Anomaly Data Set}

- CDIAC worked with Mr. P. R. Bell to produce a new time series analysis of the well-known Jones Surface Air Temperature Data Set (offered by CDIAC as NDP-020/R1). In contrast to previous analyses which were concerned with global and hemispheric mean monthly temperature time series, we have generated a set of time series subsets and accompanying graphics that look at mean monthly temperature time series for bands of $15^{\circ}$ latitude by $90^{\circ}$ longitude. Mr. Bell has conducted a preliminary analysis of these time series and found some evidence to support his hypothesis that ocean tides are a causal factor in long-term temperature trends. 


\section{Future NDPs}

- Received U.S.S.R. 6-hr synoptic climate data.

- Completion of the NDP that will contain Rasmussen and Khalil's continuous methane measurements from Cape Meares, Oregon was celayed until a paper being prepared by Khalil and Francis Moraes on these data has been published and CDIAC receives the last three years of this record. This record constitutes one of, if not the longest, methane record in the world with over 100,000 flask measurements.

- Following encouragement from Roger Dahlman to archive their data and make them available through CDIAC as NDPs, CDIAC staff spoke with Sandra Brown, Lewis Iverson, Charles Hall, and Will Ravenscroft concerning their data sets and the prospect of making them available as NDPs.

- In response to findings from the data survey conducted as part of the separate Quantitative Links support project (see p. 73), made arrangements to obtain aerosol optical depth measurements from Rattlesnake Mountain Observatory in southeastern Washington. These data will be forwarded to CDIAC in early summer of FY 1992 and packaged during the fall of FY 1992.

- CDIAC will probably package John Richards and Betsy Flint's (Duke University) data base on land use changes and resulting carbon losses/sequestering for Southeast Asia as an NDP during FY 1993. Data were recently received data for 15 zones ( 3 countries-Bangladesh, Thailand, and Myanmar) as Lotus spreadsheets. Remaining data are expectcd in early fall of 1992. Spreadsheets are presently being reviewed to gain a better understanding of their contents, and ARC/INFO export coverage files provided by David Skole (University of New Hampshire) are being reviewed to determine whether the coverages incorporate the most recent version of the data base. CDIAC's preliminary plans are to offer this data base as ARC/NFO export files, flat ASCII files, and as LOTUS files.

- An NDP containing 3-hourly weather data from 223 stations in the former U.S.S.R. is being prepared.

Three hourly observations of some 22 meteorological variables (e.g., temperature, precipitation amount and type, cloud amount and type) from 223 former U.S.S.R. stations over the period 1966-1986 are being quality assured and documented.

\section{Fossil Fuel Data Base}

- Tom Boden and Gregg Marland generated global, regional, and national $\mathrm{CO}_{2}$ emission estimates from fossil fuel burning, cement production, and gas flaring for 1950-1990. The estimates will be presented in Trends ' 93 and are being documented as an NDP (see NDP section). These emission estimates are derived from UN energy statistics, cement production estimates from the U.S. Bureau of Mines, and gas flaring estimates provided by the UN and DOE's Energy Information Administration. These statistics are thoroughly checked before emission estimates are generated.

- The emission estimates show that global total $\mathrm{CO}_{2}$ emissions reached 6.1 gigatons of carbon in 1990 , an increase of $1.2 \%$ over 1989 and continuing an increasing trend that has prevailed since 1983 . The three largest emitters in 1990-the U.S., U.S.S.R., and China-account for approximately half of the world's emissions from fossil fuel consumption. U.S. emissions reached 1.31 gigatons of carbon in 1990 (5.26 metric tons of carbon per person), the same as 1989 emissions (given the confidence limits of the data). This marked the first year since 1982-83 that U.S. emissions did not increase.

Gregg Marland presented the 1950-1989 emission estimates at the advanced NATO workshop in Italy. This Marland and Boden paper will be published in a proceedings book. These 1950-1989 emission estimates were also presented in Trends' 91. 
Table 7. Numeric data package/computer model package requests

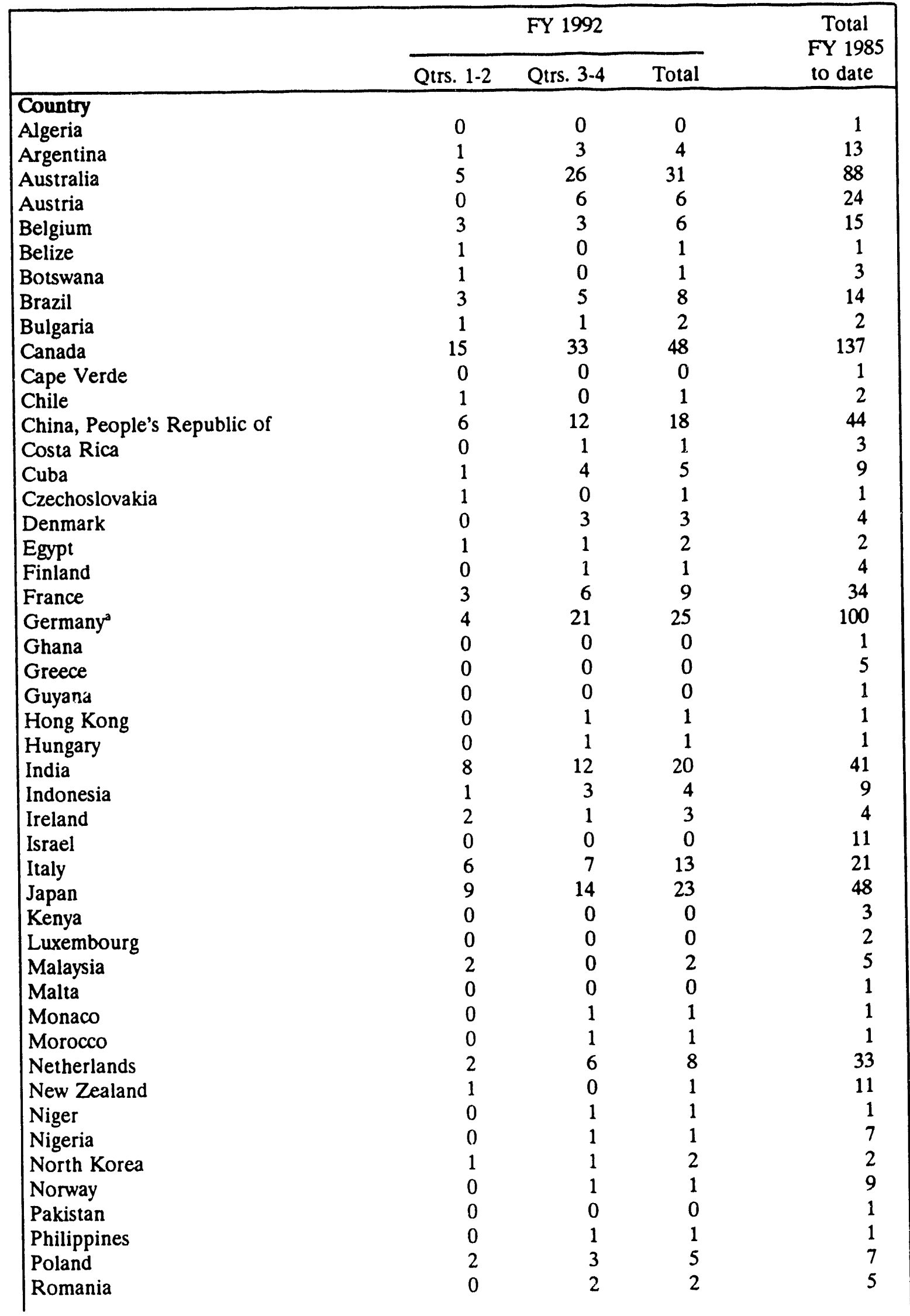


Table 7. (continued)

\begin{tabular}{|c|c|c|c|c|}
\hline & \multicolumn{3}{|c|}{ FY 1992} & \multirow{2}{*}{$\begin{array}{l}\text { Total } \\
\text { FY } 1985 \\
\text { to date }\end{array}$} \\
\hline & Qtrs. 1-2 & Qtrs. 3-4 & Total & \\
\hline \multicolumn{5}{|l|}{ Country } \\
\hline Russia & 5 & 3 & 8 & 29 \\
\hline Singapore & 0 & 1 & 1 & 3 \\
\hline South Africa & 0 & 2 & 2 & 7 \\
\hline South Korea & 0 & 5 & 5 & 7 \\
\hline Spain & 2 & 2 & 4 & 10 \\
\hline Sri Lanka & 0 & 2 & 2 & 2 \\
\hline Sweden & 1 & 3 & 4 & 11 \\
\hline Switzerland & 1 & 5 & 6 & 27 \\
\hline Taiwan & 0 & 2 & 2 & 3 \\
\hline Thailand & 0 & 0 & 0 & 2 \\
\hline Turkey & 0 & 0 & 0 & 3 \\
\hline United Kingdom & 11 & 19 & 30 & 112 \\
\hline United States & 148 & 434 & 582 & 2,008 \\
\hline Uruguay & 0 & 0 & 0 & 1 \\
\hline Vietnam & 0 & 0 & $\underline{0}$ & 2 \\
\hline Total & 249 & 661 & 910 & 2,962 \\
\hline
\end{tabular}

ancludes both German Democratic Republic and Federal Republic of Germany before FY 1991. 
Table 8. Requests for documents + tape/diskette and documents only

FY 1992

\begin{tabular}{|c|c|c|c|c|c|}
\hline & & $\begin{array}{l}\text { Documents + } \\
\text { Tape/Diskette }\end{array}$ & $\begin{array}{l}\text { Documents } \\
\text { Only }\end{array}$ & Total & $\begin{array}{l}\text { FY85 } \\
\text { to Date } \\
\text { Total }\end{array}$ \\
\hline NDP-001 & $\begin{array}{l}\text { Atmospheric } \mathrm{CO}_{2} \text { Concentrations } \\
\text { Mauna Loa Observatory, Hawaii, } \\
\text { 1958-1983 }\end{array}$ & \multicolumn{2}{|c|}{ No longer distributed } & & 68 \\
\hline NDP-001/R1 & $\begin{array}{l}\text { Atmospheric } \mathrm{CO}_{2} \text { Concentrations- } \\
\text { Mauna Loa Observatory, Hawaii, } \\
\text { 1958-1986 }\end{array}$ & 69 & 7 & 76 & 341 \\
\hline NDP-002 & $\begin{array}{l}\text { Tree Ring Chronology Indexes and } \\
\text { Reconstructions of Precipitation } \\
\text { in Central Iowa, U.S.A. }\end{array}$ & 21 & 3 & 24 & 90 \\
\hline NDP-003 & $\begin{array}{l}\text { Surface Air Temperature Anomalies } \\
\text { for the Northern Hemisphere, } \\
\text { 1881-present }\end{array}$ & \multicolumn{2}{|c|}{ No longer distributed } & & 84 \\
\hline NDP-003/R1 & $\begin{array}{l}\text { Global Surface Air Temperature } \\
\text { Variations } 1851-1984\end{array}$ & 62 & 6 & 68 & 248 \\
\hline NDP-004 & $\begin{array}{l}\text { Transient Tracers in the Oceans } \\
\text { (TTO)-Hydrographic Data and } \\
\text { Carbon Dioxide Systems }\end{array}$ & \multicolumn{2}{|c|}{ No longer distributed } & & 28 \\
\hline NDP-004/R1 & $\begin{array}{l}\text { Transient Tracers in the Oceans } \\
\text { (TTO)-Hydrographic Data and } \\
\text { Carbon Dioxide Systems with } \\
\text { Revised Carbon Chemistry Data }\end{array}$ & 26 & 1 & 27 & 90 \\
\hline NDP-005 & $\begin{array}{l}\text { Atmospheric } \mathrm{CO}_{2} \text { Concentrations } \\
\text { The NOAA/GMCC Flask and } \\
\text { Continuous Sampling Network }\end{array}$ & 32 & 6 & 38 & 156 \\
\hline NDP-006 & $\begin{array}{l}\text { Production of } \mathrm{CO}_{2} \text { from Fossil Fuel } \\
\text { Burning by Fuel Type, } 1860-1982\end{array}$ & 47 & 11 & 58 & 261 \\
\hline NDP-007 & $\begin{array}{l}\text { Atmospheric } \mathrm{CO}_{2} \text { Concentrations- } \\
\text { The CSIRO (Australia) Monitoring } \\
\text { Program from Aircraft for 1972-1981 }\end{array}$ & 11 & 3 & 14 & 95 \\
\hline NDP-008 & $\begin{array}{l}\text { Seasonal Tropospheric and Strato- } \\
\text { spheric Temperature Anomalies for } \\
\text { 1958-1984 }\end{array}$ & \multicolumn{2}{|c|}{ No longer distributed } & & 52 \\
\hline NDP-008/R1 & $\begin{array}{l}\text { Annual and Seasonal Global } \\
\text { Temperature Anomalies in the } \\
\text { Troposphere and Low Stratosphere, } \\
\text { 1958-Summer } 1986\end{array}$ & 30 & 5 & 35 & 116 \\
\hline
\end{tabular}


Table 8. (continued)

FY 1992

\begin{tabular}{|c|c|c|c|c|c|}
\hline & & $\begin{array}{l}\text { Documents }+ \\
\text { Tape/Diskette }\end{array}$ & $\begin{array}{l}\text { Documents } \\
\text { Only }\end{array}$ & Total & $\begin{array}{l}\text { FY85 } \\
\text { to Date } \\
\text { Total }\end{array}$ \\
\hline NDP-009 & $\begin{array}{l}\text { Growth and Chemical Responses to } \\
\mathrm{CO}_{2} \text { Enrichment-Virginia Pine } \\
\text { (Pinus virginiana Mill.) }\end{array}$ & 12 & 6 & 18 & 80 \\
\hline NDP-010 & $\begin{array}{l}\text { Atmospheric } \mathrm{CO}_{2} \text { Concentrations- } \\
\text { The CSIRO Program: Surface Data } \\
\text { for Cape Grim, Tasmania }\end{array}$ & 13 & 4 & 17 & 87 \\
\hline NDP-011 & $\begin{array}{l}\text { Global Paleoclimatic Data for } \\
6000 \text { Yr B.P. }\end{array}$ & 42 & 9 & 51 & 183 \\
\hline NDP-012 & $\begin{array}{l}\text { Climatic Data for Northern } \\
\text { Hemisphere Land Areas, } \\
1851-1980\end{array}$ & 28 & 19 & 47 & 217 \\
\hline NDP-013 & $\begin{array}{l}\text { Volcanic Loading: The Dust } \\
\text { Veil Index }\end{array}$ & 35 & 5 & 40 & 150 \\
\hline NDP-014 & $\begin{array}{l}\text { Solar Records: The Wolf } \\
\text { Sunspot Index and Umbral/ } \\
\text { Penumbral Ratio }\end{array}$ & 39 & 3 & 42 & 139 \\
\hline NDP-015 & $\begin{array}{l}\text { Surface Air Temperature } \\
\text { Anomalies for the Northern } \\
\text { Hemisphere: The Russian } \\
\text { Dataset }\end{array}$ & 10 & 4 & 14 & 84 \\
\hline NDP-016 & $\begin{array}{l}\text { Climatic Data for Selected } \\
\text { U.S. and Canadian Stations, } \\
1941-1980\end{array}$ & 24 & 3 & 27 & 122 \\
\hline NDP-017 & $\begin{array}{l}\text { Major World Ecosystem Com- } \\
\text { plexes Ranked by Carbon in } \\
\text { Live Vegetation: A Database }\end{array}$ & 79 & 15 & 94 & 309 \\
\hline NDP-018 & $\begin{array}{l}\text { Worldwide Organic Soil } \\
\text { Carbon and Nitrogen Data }\end{array}$ & 45 & 16 & 61 & 219 \\
\hline NDP-019 & $\begin{array}{l}\text { United States Historical } \\
\text { Climatology Network (HCN) } \\
\text { Serial Temperature and } \\
\text { Precipitation Data }\end{array}$ & \multicolumn{2}{|c|}{ No Longer Distributed } & & 170 \\
\hline NDP-019/R1 & $\begin{array}{l}\text { United States Historical } \\
\text { Climatology Network (HCN) } \\
\text { Serial Temperature and } \\
\text { Precipitation Data }\end{array}$ & 50 & 29 & 79 & 239 \\
\hline
\end{tabular}


Table 8. (continued)

FY 1992

\begin{tabular}{|c|c|c|c|c|c|}
\hline & & $\begin{array}{l}\text { Documents }+ \\
\text { Tape/Diskette }\end{array}$ & $\begin{array}{l}\text { Documents } \\
\text { Only }\end{array}$ & Total & $\begin{array}{l}\text { FY85 } \\
\text { to Date } \\
\text { Total }\end{array}$ \\
\hline NDP-020 & $\begin{array}{l}\text { A Global Grid Point Surface } \\
\text { Air Temperature Data Set: } \\
\text { 1851-1984 }\end{array}$ & \multicolumn{2}{|c|}{ No longer distributed } & & 174 \\
\hline NDP-0201R1 & $\begin{array}{l}\text { An Updated Global Grid Point } \\
\text { Surface Air Temperature } \\
\text { Anomaly Data Set: } 1851-1990\end{array}$ & 90 & 27 & 117 & 118 \\
\hline NDP-021 & $\begin{array}{l}\text { Historical Sunshine Data in } \\
\text { the United States }\end{array}$ & \multicolumn{2}{|c|}{ No longer distributed } & & 93 \\
\hline NDP-021/R1 & $\begin{array}{l}\text { Historical Sunshine and Cloud } \\
\text { Data in the United States }\end{array}$ & 56 & 12 & 68 & 96 \\
\hline NDP-022 & $\begin{array}{l}\text { Global and Hemispheric Annual } \\
\text { Temperature Variations Between } \\
1861 \text { and } 1984\end{array}$ & \multicolumn{2}{|c|}{ No longer distributed } & & 56 \\
\hline NDP-022/R1 & $\begin{array}{l}\text { Global and Hemispheric Annual } \\
\text { Temperature Variations Between } \\
1861 \text { and } 1984\end{array}$ & 62 & 11 & 73 & 231 \\
\hline NDP-023 & $\begin{array}{l}\text { Seasonal and Annual Radiosonde } \\
\text { Ozone Deviations, 1958-1985 }\end{array}$ & 34 & 6 & 40 & 124 \\
\hline NDP-024 & $\begin{array}{l}\text { A Global Planktonic Foraminifera } \\
\text { Base for Evaluation of the } \\
\text { Stability of Low-Latitude Sea } \\
\text { Surface Temperatures }\end{array}$ & \multicolumn{2}{|c|}{ No longer distributed } & & 8 \\
\hline NDP-025 & $\begin{array}{l}\text { Monthly Mean Pressure Reconstructions } \\
\text { For Europe }(1780-1980) \text { and North } \\
\text { America }(1858-1980)\end{array}$ & as & 4 & 23 & 77 \\
\hline NDP-026 & $\begin{array}{l}\text { Climatological Data for Clouds } \\
\text { Over the Globe from Surface } \\
\text { Observations }\end{array}$ & 18 & 13 & 31 & 144 \\
\hline NDP-027 & $\begin{array}{l}\text { GEOSECS Atlantic, Pacific, Indian } \\
\text { and Mediterranean Radiocarbon } \\
\text { Data }\end{array}$ & 16 & 4 & 20 & 64 \\
\hline NDP-028 & $\begin{array}{l}\text { Carbonate Chemistry of the Weddell } \\
\text { Sea }\end{array}$ & 17 & 2 & 19 & 45 \\
\hline NDP-029 & $\begin{array}{l}\text { Carbonate Chemistry of the North } \\
\text { Pacific Ocean }\end{array}$ & 18 & 2 & 20 & 53 \\
\hline
\end{tabular}


Tablc 8. (continued)

FY 1992

\begin{tabular}{|c|c|c|c|c|c|}
\hline & & $\begin{array}{l}\text { Documents + } \\
\text { Tape/Diskette }\end{array}$ & $\begin{array}{l}\text { Documents } \\
\text { Only }\end{array}$ & Total & $\begin{array}{l}\text { FY85 } \\
\text { to Date } \\
\text { Total }\end{array}$ \\
\hline NDP-030 & $\begin{array}{l}\text { Estimates of } \mathrm{CO}_{2} \text { Emissions from } \\
\text { Fossil Fuel Burning and Cement } \\
\text { Manufacturing Using the United } \\
\text { Nations Energy Statistics and } \\
\text { the U.S. Bureau of Mines Cement } \\
\text { Manufacturing Data }\end{array}$ & 2 & 16 & 18 & 320 \\
\hline NDP-030/R 1 & $\begin{array}{l}\text { Estimates of } \mathrm{CO}_{2} \text { Emissions from } \\
\text { Fossil Fuel Burning and Cement } \\
\text { Production: } 1950-1987\end{array}$ & \multicolumn{2}{|c|}{ No longer distributed } & & 77 \\
\hline NDP-030/R2 & $\begin{array}{l}\text { Carbon Dioxide Emissions from } \\
\text { Fossil Fuel Burning and Cement } \\
\text { Manufacturing, 1950-1988 }\end{array}$ & 81 & 6 & 87 & 97 \\
\hline NDP-030/R4 & $\begin{array}{l}\text { Global, Regional, and National } \\
\mathrm{CO}_{2} \text { Emission Estimates from } \\
\text { Fossil Fuel Burning, Cement } \\
\text { Production, and Gas Flaring: } \\
1950-1990\end{array}$ & 4 & 0 & 4 & 4 \\
\hline NDP-031 & $\begin{array}{l}\text { Average Total Snowfall Data } \\
\text { for Selected U.S. Stations }\end{array}$ & 12 & 1 & 13 & 39 \\
\hline NDP-032 & $\begin{array}{l}\text { Antarctic Surface Temperature } \\
\text { and Pressure Data }\end{array}$ & 25 & 3 & 28 & 82 \\
\hline NDP-033 & $\begin{array}{l}\text { Atmospheric } \mathrm{CO}_{2} \text { Concentrations } \\
\text { Derived from Flask Samples } \\
\text { Collected at U.S.S.R-Operated } \\
\text { Sampling Sites }\end{array}$ & 15 & 6 & 21 & 21 \\
\hline NDP-034 & $\begin{array}{l}\text { Atmospheric } \mathrm{CO}_{2} \text { Concentrations - } \\
\text { The Canadian Background Air } \\
\text { Pollution Monitoring Network }\end{array}$ & 15 & 1 & 16 & 40 \\
\hline NDP-035 & $\begin{array}{l}\text { A Global Geographic Information } \\
\text { System Data Base of Storm } \\
\text { Occurrences and Other Climatic } \\
\text { Phenomena Affecting Coastal Zones }\end{array}$ & 61 & 10 & 71 & 84 \\
\hline NDP-036 & $\begin{array}{l}\text { Indian Ocean Radiocarbon: Data } \\
\text { from the Indigo } 1,2 \text {, and } 3 \text { Cruises }\end{array}$ & 25 & 3 & 28 & 49 \\
\hline NDP-037 & $\begin{array}{l}\text { Carbon Dioxide Enrichment: Data on } \\
\text { the Response of Cotton to Varying } \\
\mathrm{CO}_{2} \text {, Irrigation, and Nitrogen }\end{array}$ & 5 & 0 & 5 & 5 \\
\hline
\end{tabular}


Table 8. (continued)

FY 1992

\begin{tabular}{|c|c|c|c|c|c|}
\hline & & $\begin{array}{l}\text { Documents + } \\
\text { Tape/Diskette }\end{array}$ & $\begin{array}{l}\text { Documents } \\
\text { Only }\end{array}$ & Total & $\begin{array}{l}\text { FY85 } \\
\text { to Date } \\
\text { Total }\end{array}$ \\
\hline NDP-038 & $\begin{array}{l}\text { Atmospheric Methane Concen- } \\
\text { trations - the NOAA/CMDL Global } \\
\text { Cooperative Flask Sampling } \\
\text { Network, } 1983-1988\end{array}$ & 37 & 10 & 47 & 83 \\
\hline NDP-039 & $\begin{array}{l}\text { Two Long-Term Instrumental } \\
\text { Climatic Data Bases of the } \\
\text { People's Republic of China }\end{array}$ & 39 & 5 & 44 & 44 \\
\hline NDP-041 & $\begin{array}{l}\text { The Global Historical Climatology } \\
\text { Network: Long-Term Monthly } \\
\text { Temperature, Precipitation, Sea } \\
\text { Level Pressure, and Station Pressure } \\
\text { Data }\end{array}$ & 0 & 1 & 1 & 1 \\
\hline NDP-042 & $\begin{array}{l}\text { United States Historical Climatology } \\
\text { Network Daily Temperature and } \\
\text { Precipitation Data }\end{array}$ & 41 & 19 & 60 & 60 \\
\hline CMP-002 & $\begin{array}{l}\text { The IEA/ORAU Long-Term Global } \\
\text { Energy } \mathrm{CO}_{2} \text { Model }\end{array}$ & 0 & 0 & 0 & 68 \\
\hline $\begin{array}{l}\text { CMP-002/ } \\
\text { PC }\end{array}$ & $\begin{array}{l}\text { The IEA/ORAU Long-Term Global } \\
\text { Energy } \mathrm{CO}_{2} \text { Model: Personal } \\
\text { Computer Version A\&4PC }\end{array}$ & \multicolumn{2}{|c|}{ No longer distributed } & & 267 \\
\hline $\begin{array}{l}\text { CMP-002/ } \\
\text { PC/R1 }\end{array}$ & $\begin{array}{l}\text { The IEA/ORAU Long-Term Global } \\
\text { Enerzy } \mathrm{CO}_{2} \text { Model: Personal } \\
\text { Computer Version A84PC }\end{array}$ & 131 & 1 & 132 & 439 \\
\hline CDIAC-36 & $\begin{array}{l}\text { Trends '90: A Compendium of Data } \\
\text { on Global Change on diskette }\end{array}$ & 25 & 0 & 25 & 72 \\
\hline CDIAC-46 & $\begin{array}{l}\text { Trends '91: A Compendium of Data } \\
\text { on Global Change }\end{array}$ & 291 & 운 & 291 & 291 \\
\hline & Total & 1,814 & 318 & 2,132 & 7,054 \\
\hline
\end{tabular}




\section{Networking}

To maintain its proactive position in information management activities CDIAC strives to keep informed of current research policy and information developments and needs at the international national and local levels. In addition to the exchange of data among researchers CDIAC has taken an active role in the networking of information among government agencies industries businesses special libraries and information/data centers institutions organizations and special and public interest groups. Maintaining close professional contucts with individuals and organizations CDIAC's networking capabilities have included the dissemination of research results policy initiatives and education developments; objective technical interpretations and discussions of the technical and information management related to carbon dioxide and climate change; referrals to other individuals or organizations; and access to resources relevant to individuals' information needs. CDIAC's networking capabilities have been further enhanced by active participation in the programs and organizational administration of professional societies and associations. It has been through these networking activities that CDIAC has been able to monitor the information needs of the broad climate-change community and develop specific information products and services to help meet those needs. In carrying out its networking activities CDIAC:

- Gave an invited presentation to U.S. National Committee for CODATA's Committee for IGBP Pilot Study on Database Interfaces describing CDIAC experiences relevant to criteria the Pilot Study Committee is considering for recommendations to IGBP.

- Reviewed the Southeast Regional Climate Center's FY 1992-1994 Strategic Plan.

- Met with Ram Uppuluri (legislative assistant to Senator Albert Gore Jr.) and discussed the mission and activities of CDIAC.

- Discussed with staff at the U.S. Department of Agriculture's National Agricultural Library the listing of CDLAC in its climate change information package.

- Participated in the Data Forum on Data Management for Global Change, organized by the National Academy of Sciences Committee on Geophysical Data.

- Reviewed the U.S. Environment Protection Agency/National Oceanic \& Atmospheric Administration Global Ecosystems Database CD-ROM.

- Met with Teruyoshi Hayamizu, Deputy Director, Environmental Impact Assessment Division, Environmental Agency, Tokyo and discussed how global issues such as climate change are treated in the Japanese environmental impact assessment process relative to their treatment in the U.S. system.

- Met with John J. Easton Jr., General Counsel, U.S. Department of Energy and discussed CDIAC with emphasis on international, activities especially collaborative activities with Russian and Chinese scientists; demonstrated the Edmonds/Reilly PC model, particularly the sensitivity of $\mathrm{CO}_{2}$ emissions to fuel costs and energy efficiency.

- Met with Igor K. Larin, Chief of Laboratory, Institute of Energy Problems of Chemical Physics, Moscow and discussed trace-gas monitoring data and uncertainties in modeling trace-gas dynamics, especially hydroxyl and methane.

- Met with Osamu Kobayashi, Manager, Global Environmental Affairs Department, Global Industrial and Social Progress Research Institute, Tokyo and Kiyoyuki Minato, Senior Researcher, Environment Survey Division, Japan Automobile Research Institute, Inc., Ibaraki and discussed CDIAC's products and services and energy and $\mathrm{CO}_{2}$ emissions aspects of the transportation sector in Japan. 
- Reviewed a proposal to the National Science Foundation "Hypothesis Discovery Tools for Large Datasets."

- Attended GIS/LIS Convention in Atlanta in October. Topics discussed included Global Change Opportunities for GIS and the Utilization of GIS to Determine Climate Toxic and Atmospheric Environmental Changes.

- Presented a talk at the U.S. DOE/PRC CAS meeting in Albany, New York, September 1991.

- Attended the National Institutes for Global Environmental Change (NIGEC) methane meeting in Huntington Beach, California, January 1992.

- Attended the annual NOAA/CN.DL meeting in Boulder, Colo., March 1992.

- Fred Stoss initiated steps with Martin Marietta Energy Systems' (MMES) Central Research Library (CRL) to become a Regional Information Centre of the International Geosphere Biosphere Program (IGBP). Information Services Division (in which the CRL is located) Director, Barbara Ashdown has agreed to work with her staff in allocating time to fulfill IGBP requirements in establishing a repository status in the library. The requirements for a Regional Information Centre are to accept, retain, catalog,and promote the availability of all IGBP and related reports, and to provide copies of entire reports or extracts from reports, bulletins, and newsletter on an as-needed basis. A letter of intent was directed to appropriate IGBP channels and mmes Central Research Library was designated as an IGBPRIC. Bob Conrad and Deborah York will be the CRL contacts.

- CDIAC established contact with the U.S. Department of Commerce (NOAA) to receive preprint and final copies of the WMO/UNEP 1991 document, Scientific Assessment of Ozone Depletion: 1991.

- CDIAC reestablished contact with the American Meteorological Society to have CDIAC's information products (NDPs, CMPs), technical reports, and newsletters sent to Ann Hurreau, Editor of Meteorological \& Geophysical Abstracts.

- CDIAC's entry in the FISCAL Directory of Fee-based Research and Document Supply Services, 4th Edition: 1992/1993, was made possible by the inclusion of entries that provided information at no cost or incur no obligation to the recipient. The FISCAL Directory is nationally distributed and produced by the County of Los Angeles Public Library.

- CDIAC initiated an exchange of information and reports with the Oxford (UK) Institute for Energy Studies.

- CDIAC established contact with Fundación Sirena (Buenos Aires, Argentina), which has produced DECA, a directory of ecologists and environmental scientists in Latin America, the Caribbean, Portugal, and Spain. CDIAC and DOE documents were provided to Dr. Jorge Rabinovich, Executive Director of Fundación Sirena and Professor of Ecological Studies at Universidad de San Andrés. CDIAC received a copy of this 3000-entry directory in PC-format.

- CDIAC established contact with and distributed materials to Lorna A. Greening and Mark R. Johnson from the University of Alabama. Both are involved with NIGEC-related activities.

- CDIAC received word from Pat Murray, Librarian at the International Joint Commission's Great Lakes Regional Office in Windsor, Ontario, that the library has fallen victim to U.S. demands for its closure. When designated, the name(s) of persons responsible for the GLRO climate change programs will be given to CDIAC for receipt of future CDIAC materials. 
- CDIAC received a copy of the Directory of Long-Term Ecology Research Centers. Names of institutions and individuals were examined against entries in CDIAC's World Directory. Those names not in CDIAC's directory received information about CDIAC, including a copy of CDIAC Communications.

- Fred Stoss received a complimentary copy of Academic Press' Encyclopedia of Earth Systems Science. This reference work will be reviewed for the Special Libraries Association.

- CDIAC continued a resource exchange with the Department of Natural Resources, the Ohio Sea Grant Program, and the School of Education at the Ohio State University. Drs. Rosanne Fortner, Gary Mullins, and Vic Mayer remain quite active and their developments of Earth Systems Science (curriculum and other education programs for grades $\mathrm{K}-12$ ) are a major program emphasis. This group is among the top finalists for the grant to establish the National Environmental Education and Training Program for the U.S. EPA's Office of Environmental Education. They have used CDIAC materials extensively in their courses and grant proposals. Fred Stoss has sat on several advisory boards with Rosanne Fortner, and Gary Mullins served as a Project Officer for the Clearing the Air Program on a Department of Interior subcontract for which Fred Stoss served as Principle Investigator, while employed in Rochester, New York.

- CDIAC received information and a call for papers from Dr. Otto Hutzinger (University of Bayreuth, Germany) for the ECOINFOMRA '92: 2nd International Congress and Exhibition on Environmental Information and Communication. There were no categories for global environmental studies, climate or global change. The topics covered included chemical waste management, chemical hazards, and toxicology, and the "priority" air pollutants. A call to the program office indicated that the conference themes had been pre-selected and there was no possibility to add additional topics to the conference.

- CDIAC and other ORNL ESD staff met with Derek Winstanley, DOE scientist appointed to become the next Director of the National Acid Precipitation Assessment Program (NAPAP). Derek met to discuss CDIAC's data management activities and services and products that might serve as models for future NAPAP needs. He was extremely interested in the public outreach and environmental education activities of CDIAC, and how are materials are distributed to an education community.

- CDIAC discussed with representatives from the Discovery Channel the use of CDIAC materials for an upcoming laser video disk that they are preparing on the topic of Earth sciences and climate change.

- CDIAC requested materials from DOE's Office of Scientific and Technical Information (OSTI) about the Energy Technology Data Exchange (ETDE) and the International Energy Agency's new bibliographic effort, Greenhouse Gases Bulletin, for inclusion in a future issue of CDLAC Communications.

- CDIAC provided Janet Wright (National Agricultural Library, USDA) with CDIAC/DOE materials to be used in the updated version of the NAL's Global Change Information Packet.

- Fred Stoss participated as a judge for the 1992 Southern Appalachian Science and Engineering Fair (Knoxville), the second place winner in the Junior Biology Category, which Fred judged, was an entry on the effects of $\mathrm{CO}_{2}$ enrichment on plants. Dale Kaiser, representing the American Meteorological Society, served as a judge for the National Science Fair competition in Nashville.

- Fred Stoss was invited to attend the 1992 meeting of the EPA and NOAA Librarians. This first meeting of professional library staff from these two agencies was called to increase the effectiveness of sharing resources and discuss problems and issues jointly shared by the libraries at the EPA and NOAA. Topics included chemical wastes and hazards, climate change, and environmental data. David Blockstein from the Committee for the National Institutes for the Environment provided a keynote talk. 
- CDIAC received copies of the U.S. Forest Service's (USDA) Fact Sheets describing ongoing research projects conducted by the USFS (1-page nontechnical overviews), which describe the R\&D mandate of the USFS, and progress to date on the research.

- CDIAC initiated contact with ISAR (the Institute for Soviet and American Relations). CDIAC obtained a 200-name list of corporations and other organizations involved with ISAR's Environmental Activities in the former Soviet Union.

- Fred Stoss was acknowledged in Gale Research Corporation's Environmental Source Book for his advice and assistance in preparation of this reference book.

- CDIAC provided Bill Purvis (National Coal Association) with a copy of the DOE Research Summary No. 14 for their reproduction and distribution to members of the NCA.

- CDLAC received from the University of California - Davis, National Institute for Global Environmental Change, a copy of the Global Systems Science, Vol. 1. A Planet Under Siege: The Problem of Global Warming. This report has been prepared as a curriculum for secondary teaching of the concepts related to climate change. The report was prepared by staff at the Lawrence Hall of Science at the University of California at Berkeley.

- CDIAC staff met with Martha Maiden (NASA DAAC Manager) and Robert Price (NASA EOSDIS Chief Scientist) about how CDIAC approaches its information analysis center role. Maiden and Price visited ORNL in association with the newly named National Aeronautics and Space Administration (NASA) Earth Observing System (EGOS) Data and Information System (DAIS) Distributed Active Archive Center (DAAC) at ORNL. The ORNL DAAC will be responsible for data on trace gas fluxes.

- CDIAC staff met with Dr. S. C. Majumdar (Joint Advisor to the Director General, Council of Scientific and Industrial Research, New Delhi, India, and Head of the United Nations Research Development Program in India) concerning CDIAC, global warming, and $\mathrm{CO}_{2}$ emissions. Majumdar is administratively responsible for technology transfer, global warming, Interface for Nonresident Indian Scientists \& Technologists (INRIST), and other UN-sponsored programs in India.

- CDIAC staff met with Professor Tadashi Matsunaga and Dr. Chikashi Nakamura (Tokyo University of Agriculture and Technology) and Dr. Hiroyuki Takano (Onoda Cement Co., Ltd., Osaka) concerning $\mathrm{CO}_{2}$ emissions from the cement industry and the potential for $\mathrm{CO}_{2}$ recovery.

- CDIAC staff met with William C. Clark (Assistant Director of the Center for Science and International Affairs, Harvard University) concerning information issues related to climate change (needed interdisciplinary data sets; trends towards key scie tific papers not appearing as published literature but rather as conference and informal working drafts, such as in the Global Warming Potential debate; and implications for availability to the research community).

- CDIAC staff met with Kiyoyuki Minato (Senior Researcher with the Japan Automobile Research Institute) to discuss his institute's estimates of $\mathrm{CO}_{2}$ emissions from various motor vehicles, Japanese industrial research into approaches for reducing pollutant emissions from the transportation sector, and CDIAC's data products that could be of use to the institute. Minato visited CDIAC in 1991. He presented an update on Japanese $\mathrm{CO}_{2}$ emissions, a valuable source of data for verifying the data on $\mathrm{CO}_{2}$ emissions that CDIAC derives from other sources, principally the United Nations.

- CDIAC hosted a visit to ORNL of the National Research Council's IGBP Database Interface Committee. The committee is reviewing how CDIAC manages and produces large, integrated data sets and the significance of CDIAC's experiences for the future success of IGBP. 
- CDIAC staff presented an overview of CDIAC to Fred Henderson of GEOSAT (Norman, Okla.) during his visit to ORNL on September 10, 1992.

- CDIAC staff discussed information needs in the area of global climate change with a group of officials from developing countries as part of The University of Tennessee's workshop on Impact Assessment for Developing Countries.

- Reviewed a manuscript for the journal Environmental Management.

- CDIAC provided the DOE Office of Scientific and Technical Information's (OSTI) Infotech Update editor, Meg Jared, with CDIAC factsheets to enable her to describe CDIAC and its DOE-sponsored information products in future issues of this newsletter. Infotech Update is produced quarterly by OSTI to highlight DOE-sponsored products, services, systems, technologies, and activities and to provide a forum for the exchange of scientific and technical information among DOE and DOE contractor facilities. This newsletter is available only to DOE personnel and DOE contractors.

- CDIAC exchanged materials with the U.S. national contact for CADDET (Centre for the Analysis and Dissemination of Demonstrated Energy Technologies), which is a program of the International Energy Agency. The U.S. CADDET office is located at Oak Ridge National Laboratory. The September issue of CADDET's IEA Heat Pump Centre Newsletter contained a series of articles discussing the role of heat pump and CFC-alternative technologies in the context of global climate change. Materials about CDIAC will be forwarded to the appropriate CADDET newsletters for inclusion in future issues.

- CDIAC provided Theodore W. Kheel, Publisher of the Earth Summit Times, with copies of CDIAC publications. This newspaper was published daily before the UNCED conference. After the conference, the newspaper's fate was less certain, and they began a weekly distribution. No further word has been received about this periodical. Kheel is a past publisher of the NY Daily News.

- CDIAC established contact with Claire S. Hanson, Co-chair of the ARCUS (Arctic Research Consortium of the U.S.) Data and Information Resources Working Group. CDIAC was listed in a pamphlet, "Guide to Selected Arctic Information and Data Research."

- CDIAC was included in a primer on climate change prepared by Oceanic, the Ocean Network Information Center. Information from CDIAC focused on several of our NDPs.

- CDIAC established contact with Michael Williams, Media Specialist working with the United Nations Environment Programme in the newly created Information Unit on Climate Change (part of the UNEP GEMS-Global Environment Monitoring System). Various materials have been exchanged. The Information Unit on Climate Change was created to make the findings of climate change research more accessible to other researchers, decision makers, and the general public. They have prepared a series of fact sheets and videos on various technical, social, and policy aspects of climate change. Thousands of these materials have been distributed worldwide. IUCC is also sponsoring a series of seminars and training sessions in Europe, Pakistan, India, Thailand, and Egypt. Future workshop locations will be in southern Africa. An additional component being added to the program will be a presentation on how to conduct a national $\mathrm{CO}_{2}$ emission audit.

- CDIAC established contact with Ned Leonard, communications/media staffer with the Western Fuels Association, Inc. CDIAC has received several copies of their video on $\mathrm{CO}_{2}$ enrichment and plant growth, which features some of the work conducted by Sherwood Idso. CDLAC continues to receive press releases and other press and promotional literature from this special interest group.

- CDIAC provided Carol Watts (NOAA, Chair of the IWGDMGC L/IS Subgroup) with comments regarding the development of an information center concept. 
- CDIAC provided high school teachers in the DOE/TRAC at ORNL's ESD program with environmental education materials related to climate change.

- CDIAC provided Linda Musser, a research librarian at the Pennsylvania State University with information to assist her efforts in compiling an annotated bibliography of periodicals covering the topic of earth systems science (to be published in Serials Review).

- CDIAC provided Kimberly A. Carter, Librarian at the Midwest Research Institute (Kansas City, Missouri) with information about CDIAC for display at a continuing education conference sponsored by the Kansas City Metropolitan Library Network.

- CDIAC established contact with Roberto Chiarotti, Public Affairs Officer with the World Coal Institute in London. An exchange of materials was initiated.

- CDIAC provided Wendy A. Casey, Electronic Services Specialist with Cambridge Scientific Abstracts, with information about CDIAC and its information products to be considered for citation in CSA's Pollution Abstracts.

- CDIAC established contact with Norman B. Coleman, Assistant Attorney General for the State of Minnesota and principal involved in the creation of Worldview, an organization linking public affairs analysts from 40 countries on the common topic of the environment. The creation of a central information network and data base are seen as central to the Worldview mandate to inform the research and policy communities on environmentally clean technologies.

- CDIAC established contact with Cynthia Bertelsen, Project Manager with Tropical Research and Development (Gainesville, Florida).

- Kiyotaka Wasa, Deputy Director of RITE (Research Institute of Innovative Technology for the Earth, Kyoto, Japan) contacted CDIAC and facilitated the exchange of information on our respective institutions.

- Copies of Trends ' 91 were sent to the regional offices of the United Nations Environment Programme and the UN GEMS/PAC (Global Environmental Monitoring Systems/Programme Activity Centre).

- G. Dennis Campbell, Editor and Publisher of a new environmental education journal, Environmental Connections, received a copy of our newsletter and fact sheets about CDIAC and its publications.

- Discussions were held with David Schoolcraft of NOAA's National Geophysical Data Center (NGDC) concerning the use of Jerry Olson's NDP-017 (Major World Ecosystem Complexes Ranked by Carbon in Live Vegetation: A Database), which was sought for use in an electronic Global Ecosystem CD-ROM, compiled by NGDC.

- Tom Boden attended the NASA EOSDIS meeting held at Goddard Space Flight Center (GSFC) on June 2, 1992. 


\section{Quantitative Links Support}

Since FY 1990, CDIAC has received separate funding from the U.S. Department of Energy's Environmental Sciences Division to support its Quantitative Links program. Under this project, "Data Base Support to Assist in Quantifying the Link Between Changes in Atmospheric Composition and Climate Change," CDLAC provides quality-assured data sets and other information needed for quantifying the link between atmospheric chemistry, the earth's radiative balance, and climate. This project includes several tasks.

- Global Historical Climatology Network. CDIAC is compiling, documenting, and distributing a data set suitable for the study of long-term climate trends that may be related to changes in atmospheric composition. In FY 1992 numeric data package NDP-041, The Global Historical Climatology Network: Long-Term Monthly Temperature, Precipitation, Sea Level Pressure, and Station Pressure Data, (Vose, R. S., R. S. Schmoyer, P. M. Steurer, T. C. Peterson, R. Heim, T. R. Karl, and J. K. Eischeid. 1992. ORiNL'CDIAC-53. Oak Ridge National Laboratory, Oak Ridge, Tenn.) was published, in collaboration with the National Climatic Data Center. NDP-041, which presents version 1.0 of the GHCN, includes monthly surface air temperature, precipitation, and atmospheric pressure data from the World Weather Records and Monthly Climate Data for the World, supplemented with additional data from numerous other sources to increase spatial coverage. The data were quality-assured (and corrected where possible) and documented. The GHCN was then further enhanced in spatial coverage. At this point, GHCN Version 1.0 is considered one of the largest and most comprehensive long-term data sets that can be applied to the study of global climate change. It has been constructed using records from numerous preexisting sources, thus consolidating a large amount of climatic data that had previously resided in disjoint repositories. It has also been subjected to several procedures designed to refine the quality of individual station records. In addition, it will continue to evolve in the coming years. Planned improvements entail the inclusion of additional data, the correction of erroneous data, the adjustment of data inhomogeneities, the addition of new variables, and the production of gridded data sets.

Additional data sets (including Pacific atoll data for 88 stations for the period 1971-1990; data for Pakistani stations, primarily for the period 1930-1980; Brazilian data for the period 1910-1980; European data compiled by Germany; high-altitude data; 243 -station data from the former U.S.S.R.; and Nigerian data) were acquired for incorporation into the GHCN.

Russ Vose met with Tom Peterson of NOAA's National Climatic Data Center and Jon Eischeid of the University of Colorado's Cooperative Institute for Research in Environmental Sciences to discuss plans for the next version of the Global Historical Climatology Network data base.

- International data exchange in support of the Quantitative Links program. CDIAC is producing numeric data packages based on climate-related data sets acquired from other countries (initially, the former Soviet Union), which have been quality-assured and documented. Working under the auspices of the 1972 U.S.-U.S.S.R. Agreement on Cooperation in the Field of Environmental Protection, existing climate-related data sets residing in the former Soviet Union are identified. The data sets that are most important for the quantification of the link between radiative balance and atmospheric temperature are acquired. These data sets are quality-assured (e.g., to remove erroneous data), documented, and packaged for distribution as NDPs.

Three NDPs that have or will result from data exchanges conducted as part of this project were published by CDIAC: Atmospheric $\mathrm{CO}_{2}$ Concentrations Derived from Flask Samples Collected at U.S.S.R.Operated Sampling Sites (Brounshtein, A. M., E. V. Faber, and A. A. Shashkov. (December 1991). NDP033, ORNL/CDIAC-51, Oak Ridge National Laboratory, Oak Ridge, Tenn.), Daily Temperature and Precipitation Data for 223 U.S.S.R. Stations: 1884-1986 (March 1993), and 3-Hourly Climate Data for the U.S.S.R. (in progress). Also, global and hemispheric temperature data received from Russian 
researchers were incorporated into CDIAC's Trends '91: A Compendium of Data on Global Change (Boden, T. A., R. J. Sepanski, and F. W. Stoss. 1991. ORNL/CDIAC-46, Oak Ridge National Laboratory, Oak Ridge, Tenn.).

A follow-up visit to Obninsk and Moscow, coupled with a visit to St. Petersburg (formerly Leningrad) was completed in October 1991. Discussions were held at the following institutions: Research Institute of Hydrometeorological Information, Obninsk; Institute of Geography and Committee for Hydrometeorology, Moscow; and the State Hydrological Institute, Main Geophysical Institute, Institute for Lake Research, and Arctic and Antarctic Research Institute, St. Petersburg. Several data sets were identified for potential acquisition by CDIAC and production as CDIAC NDPs.

A trip to Brazil in June 1992 in conjunction with the United Nations Conference and Environment and Development (UNCED) yielded valuable personal contacts with a number of Brazilian centers holding data important to the Quantitative Links program: the Directoria de Hidrografia e Navegacao (DHN), Niteroi; the Observatorio Nacional, Rio de Janeiro; and the Ministerio de Aeronautica, Brasflia. Brazil was selected as a country where climate-related data are collected and maintained but are not generally available to the U.S. or international climate change communities that CDIAC serves. During this trip the role of DOE and CDIAC in global climate change research was explained, DOE and CDIAC data products and services were offered, and discussions focused on data sets potentially appropriate for inclusion in the CDIAC archives and possible packaging as NDPs.

- Russian-English translation project. CDIAC, in collaboration with the Research Institute of Hydrometeorological Information-World Data Center in Obninsk, has begun to produce a series of technical reports based on the translation into English of foreign language literature important to the Quantitative Links program. This task includes the evaluation of translation software developed for the personal computer.

Many important climate-related manuscripts, reports, and books have been published in the Russian language only, thereby limiting their usefulness to the global climate change community. The task began with the identification of important Russian language literature in the four areas identified as being of particular importance in the Quantitative Links data survey-surface energy budget, aerosols, clouds, and general circulation models. Next, a technical translator was brought to CDIAC to translate the abstracts. As part of this process, personal computer-based software for Russian-to-English translation was evaluated and a supplemental Russian-English dictionary for technical terminology was produced. Finally, the first dual-language document in this series, Selected Translated Abstracts of RussianLanguage Climate-Change Publications: I. Surface Energy Budget, was produced as a joint CDIACObninsk publication (Ravina, C. B., and M. D. Burtis. 1992. ORNL/CDIAC-57, Proceedings of RIHMI-WDC, No. 158. Oak Ridge National Laboratory, Oak Ridge, Tenn.), with appropriate credit given to both collaborating organizations. This task was conducted under the auspices of Working Group VIII of the U.S.-U.S.S.R. bilateral agreement, which has included joint publications as one of its activities. 


\section{ARM Newsletter}

- CDLAC has produced the first issue of the new newsletter, $A R M$ Outreach, with support from the DOE Global Change Research Program's Atmospheric Radiation Measurement (ARM) Program. The purpose of $A R M$ Outreach is to inform a broad audience of researchers, policymakers, managers, and educators about ARM's research and program activities. Volume 1, No. 1 of $A R M$ Outreach (pictured below) describes the ARM Program's setting within DOE; its purposes, goals, and organization; and its investigators and their research. This inaugural issue also presents summaries of two research projects conducted under the aegis of the ARM Program. 


\section{INTERNAL DISTRIBUTION}

1-2. A. W. Trivelpiece, $4500 \mathrm{~N}, \mathrm{MS}-6255$

3. D. Alvic, UT/EERC

4. L. D. Bates, K-1201, MS-7256

5. T. A. Boden, 1000 , MS- 6335

6. H. L. Boston, 1505, MS-6036

7. M. D. Burtis, 1000 , MS-6335

8. J. B. Cannon, 4500 N, MS-6189

9. P. J. Crabtree, 1000 , MS-6335

10. J. H. Cushman, 1503, MS-6352

11. R. M. Cushman, 1000 , MS-6335

12. R. C. Daniels, 1000, MS-6335

13. M. P. Farrell, 1505, MS-6038

14. D. E. Fowler, 1505, MS-6035

15. C. W. Gehrs, 1505 , MS-6036

16. P. L. Harmon, 1000 , MS- 6335

17. S. G. Hildebrand, 1505 , MS-6035

18. S. B. Jones, 1000 , MS-6335

19. D. P. Kaiser, 1000 , MS-6335

20. P. Kanciruk, 0907 MS-6490

21. D. L. Lue, 1000 , MS- 6335

22. G. Marland, 1000 , MS-6335
23. L. J. Morris, 1000 , MS-6335

24. T. R. Nelson, 1000 , MS-6335

25. D. E. Reichle, $4500 \mathrm{~N}$, MS-6253

26. R. J. Sepanski, 1000, MS-6335

27. F. E. Sharples, 1505 , MS-6036

28. D. E. Shepherd, 1000 , MS-6335

29. D. S. Shriner, 1505 , MS- 6036

30. D. E. Sill, 6026 , MS-6394

31. T. Stamm, UT/EERC

32. F. W. Stoss, 1000 , MS-6335

33. S. H. Stow, 1505 , MS- 6038

34. R. I. Van Hook, 1505, MS-6037

35. R. S. Vose, 1000 , MS-6335

36. T. W. White, 1000 , MS-6335

37-52. ESD Library, 1505, MS-6035

53. Foreign Travel

54-55. Laboratory Records Dept., 4500N, MS-6285

56. Laboratory Records Department - RC

57. Lab. Protection Div., 3546, MS-6011

58. ORNL Patent Section, 4500 N, MS-6258

59. ORNL Public Relations, 4500N, MS-6213

\section{EXTERNAL DISTRIBUTION}

60. S. Alexander, Geosciences Department, Pennsylvania State University, Iniversity Park, PA 16802

61. J. H. Allen, National Oceanic and Atmospheric Administration, National Geophysical Data Center, Code E/GC2, 325 Broadway, Boulder, CO 80303

62. A. Andersen, U.S. Department of Energy, Code EI-62, 1000 Independence Ave. SW, Washington, D.C. 20585

63. P. Andre, U.S. Department of Agriculture, National Agricultural Library, 10301 Baltimore Blvd., Beltsville, MD 20705

64. D. C. Bader, Battelle Pacific Northwest Laboratory, 901 D Street SW, Suite 900, Washington, D.C. $20024-2115$

65. R. H. Ball, U.S. Department of Energy, Code EH-3, 1000 Independence Ave. SW, Washington, D.C. 20585

66. R. C. Barry, University of Colorado, World Data Center A, Glaciology, CIRES, Campus Box 449, Boulder, CO 80309-0449

67. D. Barth, Director, Harry Reid Center for Environmental Studies, 4505 South Maryland Parkway, University of Nevada, Las Vegas, NV 89154

68. G. Barton, NOAA/NODC, Room 406, 1825 Connecticut Ave. NW, Washington, D.C. 20235

69. M. Baumgardner, Department of Agronomy, Purdue University, 1220 Potter Drive, West Lafayette, IN 47907

70. D. Bergamaschi, OES-OA/MST, Room 5081, Department of State, Washington, D.C. 20520

71. B. Bernstein, EcoAnalysis, Inc., Arcade Plaza, Suite A, 221 East Matilija, Ojai, CA 930230279 
72. D. A. Bruns, Chair, Department of Earth and Environmental Sciences, Wilkes University, Wilkes-Barre, PA 18766

73. R. Calender, Oceanographer of the Navy, U.S. Naval Observatory, 34th St. and Massachusetts Ave. NW, Washington, D.C. 20392-5101

74. M. A. Chinnery, National Oceanic and Atmospheric Administration, National Geophysical Data Center, Code E/GC2, 325 Broadway, Boulder, CO 80303

75. C. Christensen, Department of Interior, Office of Information Resources Management, 1849 C St. NW, MS-5321, Washington, D.C. 20240

76. E. Christian, U.S. Geological Survey, Information Systems Division, Reston, VA 22092

77. P. A. Crowley, Environmental Sciences Division, Office of Health and Environmental Research, ER-74, U.S. Department of Energy, Washington, D.C. 20585

78. H. Croze, Global Environment Monitoring Systems, UNEP, P. O. Box 30552, Nairobi, Kenya

79. R. C. Dahlman, Environmental Sciences Division, Office of Health and Environmental Research, ER-74, U.S. Department of Energy, Washington, D.C. 20585

80. L. DeMouy, U.S. Department of Energy, Code EI-623, 1000 Independence Ave. SW, Washington, D.C. 20585

81. W. Draeger, EROS Data Center, U.S. Geological Survey, Sioux Falls, SD 57198

82. J. J. Easton, Jr., Assistant Secretary for International Affairs and Energy Emergencies, (IE-1), Department of Energy, Washington, D.C. 20585

83. J. W. Elwood, Environmental Sciences Division, Office of Health and Environmental Research, ER-74, U.S. Department of Energy, Washington, D.C. 20585

84. L. Enomoto, NOAA/NESDIS, FB-4, Room 0110, Washington, D.C. 20233

85. G. Evans, U.S. Department of Agriculture, Global Change Program Office, 1621 N. Kent Street, Room 60LL, Arlington, VA 22209

86. R. N. Farvolden, Professor, Department of Earth Sciences, University of Waterloo, Waterloo, Ontario N2L 3G1 Canada

87. J. Fein, National Science Foundation, Atmospheric Science Division, $1800 \mathrm{G}$ St. NW, Washington, D.C. 20550

88. W. Ferrell, U.S. Department of Energy, 1000 Independence Ave. SW, Washington, D.C. 20585

89. J. Filson, National Earthquake Information Center, U.S. Geological Survey, Denver Federal Center, P.O. Box 20546, Denver, CO 80225

90 . J. F. Franklin, Bloedel Professor of Ecosystem Analysis, College of Forest Resources, University of Washington, Anderson Hall (AR-10), Seattle, WA 98195

91. A. Ghoyanlou, Technical Director, Space Systems Division, Mitre Corporation, 7525 Colshire Drive, MS Z650, McLean, VA 22102

92. S. N. Goldstein, National Science Foundation, 1800 G St. NW, Room 416, Washington, D.C. 20550

93. S. Graves, NASA, Code SED, 600 Independence Ave. SW, Washington, D.C. 20546

94. J. L. Green, National Space Science Data Center, NASA Goddard Space Flight Center, Code 630.2, Greenbelt, MD 20771

95. T. J. Gross, Environmental Sciences Division, Office of Health and Environmental Research, ER-74, U.S. Department of Energy, Washington, D.C. 20585

96. K. Hadeen, NOAA National Climatic Data Center, Federal Building, Asheville, NC 28801

97. R. C. Harriss, Institute for the Study of Earth, Oceans, and Space, Science and Engineering Research Building, University of New Hampshire, Durham, NH 03824.

98. R. Hayes, Oceanographer of the Navy, U.S. Naval Observatory, 34th St. and Massachusetts Ave. NW, Washington, D.C. 20392-5101

99. G. M. Hornberger, Professor, Department of Environmental Sciences, University of Virginia, Charlottesville, VA 22903

100. J. C. Houghton, Environmental Sciences Division, Office of Health and Environmental Research, ER-74, U.S. Department of Energy, Washington, D.C. 20585

101. G. Hunolt, NASA, Code SED, 600 Independence Ave. SW, Washington, D.C. 20546 
102. G. Y. Jordy, Director, Office of Program Analysis, Office of Energy Research, ER-30, G-226, U.S. Department of Energy, Washington, D.C. 20545.

103. J. Joyce, National Science Foundation, Atmospheric Science Division, 1800 G St. NW, Washington, D.C. 20550

104. T. R. Karl, National Clinatic Data Center, Federal Building, Asheville, NC 28801

105. D. Lauer, EROS Data Center, U.S. Geological Survey, Sioux Falls, SD 57198

106. M. S. Loughridge, National Oceanic and Atmospheric Administration, National Geophysical Data Center, Code E/GC3, 325 Broadway, Boulder, CO 80303

107. G. H. Ludwig, HC 33, Box 641, Winchester, VA 22601

108. P. Lunn, Environmental Sciences Division, Office of Health and Environmental Research, ER-74, U.S. Department of Energy, Washington, D.C. 20585

109. T. H. Mace, U.S. Environmental Protection Agency, Room 1123 West Tower, 401 M St. SW, Washington, D.C. 20460

110. L. McGoldrick, SMSRC, 600 Maryland Ave. SW, Suite 440, Washington, D.C. 20024

111. L. Meredith, USRA, 600 Maryland Ave. SW, Suite 440, Washington, D.C. 20024

112. R. E. Munn, University of Toronto, Institute for Environmental Studies, Toronto, Ontario M5S 184, Canada

113. B. Niemann, U.S. Environmental Protection Agency, PM-223X, 401 M St. SW, Washington, D.C. 20460

114. R. H. Olsen, Vice President for Research, University of Michigan, Medical Science Building II, \#5605, 1301 East Catherine Street, Ann Arbor, MI 48109-0610

115. J. T. Overpeck, National Oceanic and Atmospheric Administration, National Geophysical Data Center, Paleoclimatology Program, 325 Broadway E/EC, Boulder, CO 80303

116. A. Patrinos, Director, Environmental Sciences Division, Office of Health and Environmental Research, ER-74, U.S. Department of Energy, Washington, D.C. 20585

117. L. Pettinger, National Mapping Division, USGS/DOI, 590 National Center, Reston, VA 22092

118. I. Rasool, Lab de Meteorologie, Dynamique, Du C.N.R.S., Ecole Normale Superieure, 24 Rue Lhomond, 75231 Paris, Cedex 05 France

119. M. R. Riches, Environmental Sciences Division, Office of Health and Environmental Research, ER-74, U.S. Department of Energy, Washington, D.C. 20585

120. M. Rodon-Naveira, EPA/ORD, 401 M Street SW, Washington, D.C. 20460

121. E. Russek-Cohen, Department of Animal Sciences, University of Maryland, College Park, MD 20742

122. H. H. Shugart, Department of Environmental Sciences, Clark Hall, University of Virginia, Charlottesville, VA 22903

123. A. L. Shumbera, National Oceanic and Atmospheric Administration, WDC-A for Meteorology, National Climatic Data Center, Federal Building, MC E/CC, Asheville, NC 28801

124. P. M. Steurer, National Climatic Data Center, Database Management Branch, Federal Building, Asheville, NC 28801

125. J. Thieman, NASA/GSFC, Bldg. 26, Room 138, Code 933, Greenbelt, MD 20771

126. R. E. Thompson, Department of Agriculture, National Agricultural Library, 10301 Baltimore Blvd./5th Floor, Beltsville, MD 20705

127. P. F. Uhlir, Assistant Executive Director, Commission on Physical Sciences, Mathematics, and Applications, National Research Council, 2101 Constitution Avenue NW, Washington, D.C. 20418

128. C. Watts, NOAA/Library, 6009 Executive Blvd., Rockville, MD 20852

129. G. B. Wiersma, Dean, College of Forest Resources, Maine Agricultural Experiment Station, 202 Nutting Hall, University of Maine, Orono, ME 04469

130. R. Williams, U.S. Department of Agriculture, OIRM - Room 414-W, Washington, D.C. 20250

131. G. Withee, NOAA/NODC, Room 506, 1825 Connecticut Ave. NW, Washington, D.C. 20235 
132. F. J. Wobber, Environmental Sciences Division, Oiffice of Health and Environmental Research, ER-74, U.S. Department of Energy, Washington, D.C. 20585

133. L. Wolf, National Academy of Sciences, 2001 Wisconsin Ave. NW, Harris Building 372, Washington, D.C. 20007

134. J. Young, Commission on Physical Sciences, Mathematics, and Applications, National Research Council, 2101 Constitution Avenue NW, Washington, D.C. 20418

135. Office of Assistant Manager for Energy Research and Development, Oak Ridge Field Office, P.O. Box 2001, U.S. Department of Energy, Oak Ridge, TN 37831-8600

136-37. Office of Scientific and Technical Information, P.O. Box 62, Oak Ridge, TN 37831 

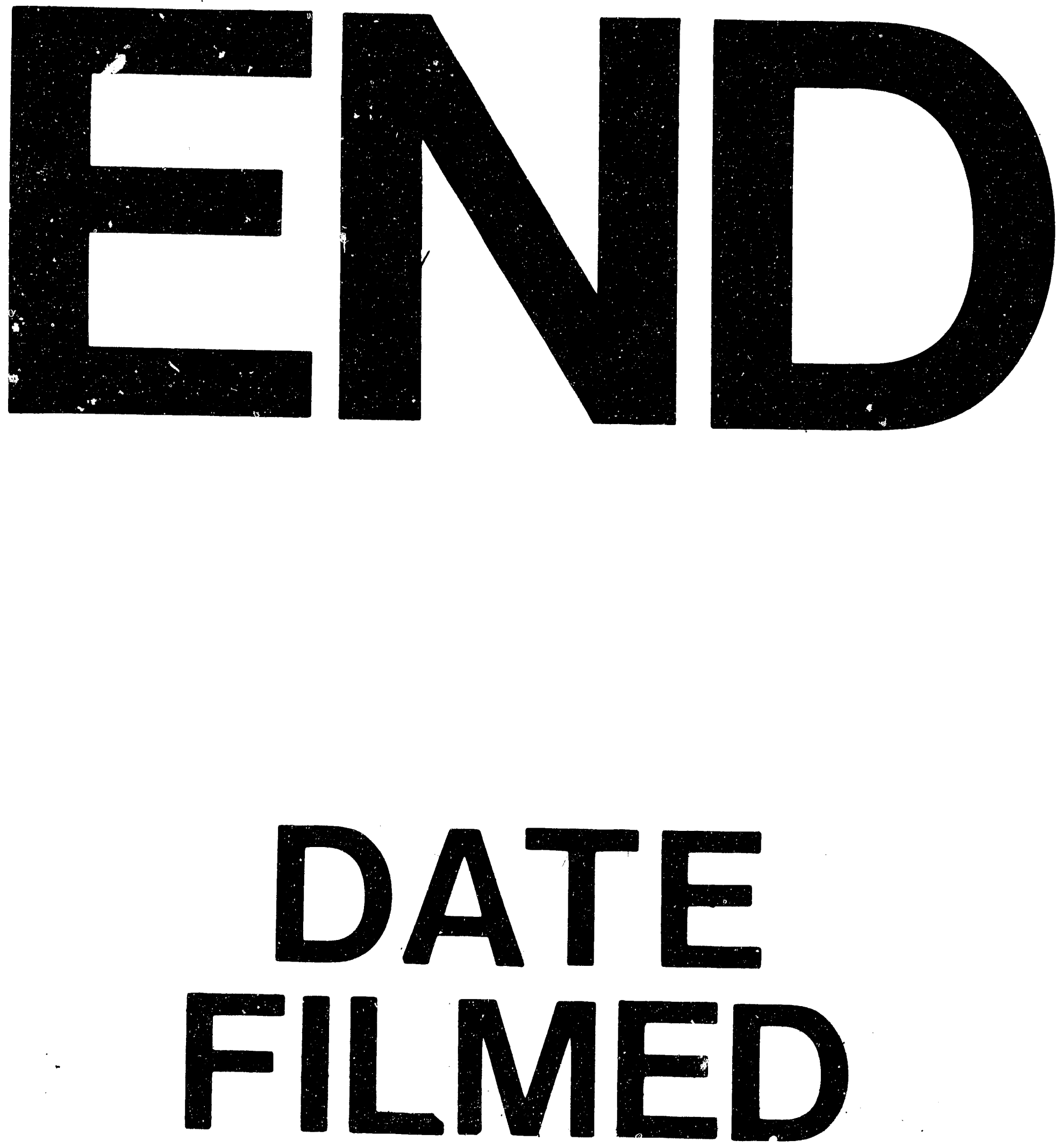

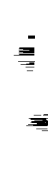

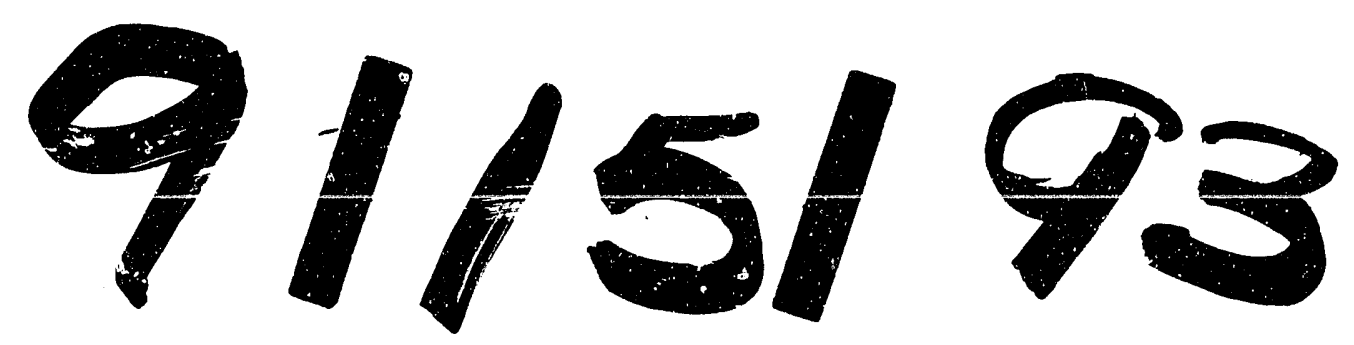


